Draft Version July 30, 2018

Preprint typeset using $\mathrm{LAT}_{\mathrm{E} X} \mathrm{X}$ style emulateapj v. 08/22/09

\title{
THE COEVOLUTION OF SUPERMASSIVE BLACK HOLES AND MASSIVE GALAXIES AT HIGH REDSHIFT
}

\author{
A. Lapi ${ }^{1,2}$, S. Raimundo ${ }^{1}$, R. Aversa ${ }^{1}$, Z.-Y. Cai ${ }^{1,3}$, M. Negrello ${ }^{4}$, A. Celotti ${ }^{1,5,6}$, G. De Zotti ${ }^{1,4}$, L. Danese ${ }^{1,6}$ \\ Draft version July 30, 2018
}

\begin{abstract}
We exploit the recent, wide samples of far-infrared (FIR) selected galaxies followed-up in X rays and of X-ray/optically selected active galactic nuclei (AGNs) followed-up in the FIR band, along with the classic data on AGN and stellar luminosity functions at high redshift $z \gtrsim 1.5$, to probe different stages in the coevolution of supermassive black holes (BHs) and host galaxies. The results of our analysis indicate the following scenario: (i) the star formation in the host galaxy proceeds within a heavily dust-enshrouded medium at an almost constant rate over a timescale $\lesssim 0.5-1 \mathrm{Gyr}$, and then abruptly declines due to quasar feedback; over the same timescale, (ii) part of the interstellar medium loses angular momentum, reaches the circum-nuclear regions at a rate proportional to the star formation and is temporarily stored into a massive reservoir/proto-torus wherefrom it can be promptly accreted; (iii) the $\mathrm{BH}$ grows by accretion in a self-regulated regime with radiative power that can slightly exceed the Eddington limit $L / L_{\mathrm{Edd}} \lesssim 4$, particularly at the highest redshifts; (iv) for massive BHs the ensuing energy feedback at its maximum exceeds the stellar one and removes the interstellar gas, thus stopping the star formation and the fueling of the reservoir; (v) afterwards, if the latter has retained enough gas, a phase of supply-limited accretion follows exponentially declining with a timescale of about $2 e$-folding times. We also discuss how the detailed properties and the specific evolution of the reservoir can be investigated via coordinated, high-resolution observations of starforming, strongly-lensed galaxies in the (sub-)mm band with ALMA and in the X-ray band with Chandra and the next generation X-ray instruments.
\end{abstract}

Subject headings: galaxies: formation - galaxies: evolution - galaxies: elliptical - galaxies: high redshift - quasars: general

\section{INTRODUCTION}

The joint formation and evolution of galaxies and active galactic nuclei/quasars (AGNs/QSOs) is still a major problem in astrophysics and cosmology. One of the key points has been the discovery, via stellar/gas dynamics and photometric observations, that local elliptical-type galaxies (ETGs) and massive bulges exhibit at their centers a massive dark object (MDO) endowed with a mass $M_{\bullet} \sim 10^{-3} M_{\star}$ proportional to the mass in old stars or to the $K$-band luminosity (Kormendy \& Richstone 1995; Magorrian et al. 1998; Gebhardt et al. 2000a; Marconi \& Hunt 2003; Häring \& Rix 2004; McLure \& Dunlop 2004; Ferrarese \& Ford 2005; Graham 2007; Sani et al. 2011; Beifiori et al. 2012; McConnell \& Ma 2013; Kormendy \& Ho 2013).

Indeed, the hypothesis that MDOs are the black hole $(\mathrm{BH})$ remnants of a past QSO activity was one of the major motivation for their searches, following the suggestion that QSOs are powered by gas accretion onto supermassive BHs (Salpeter 1964; Zel'dovich \& Novikov 1964) and the integral argument presented by Soltan (1982). Salucci et al. (1999) demonstrated that the mass function of MDOs in local ETGs and bulges, derived on the basis of the local $M_{\bullet}-M_{\star}$ correlation, is very close to the mass function of the accreted matter estimated by exploiting the redshift-dependent AGN/QSO luminosity function, if the paradigm of accretion disc onto a BH (with reasonable matter-radiation conversion efficiency and limiting luminosity) is assumed. Moreover, from the analysis of the orbits for a number of individual stars in the central region of our Galaxy the presence of a supermassive BH with mass $M_{\mathrm{BH}} \approx 4 \times 10^{6} M_{\odot}$ has been established at a very high confidence level (Genzel et al. 2010). Once MDOs are identified as BHs, the $M_{\bullet}-M_{\star}$ relation translates into a relationship between the $\mathrm{BH}$ mass $M_{\mathrm{BH}}$ and the stellar mass $M_{\star}$, suggesting that the star formation on large (i.e., kpc) scale in (proto-)ETGs and the accretion onto the BHs on much smaller (sub-pc) scale must in a way talk to each other (e.g., Alexander \& Hickox 2012).

The luminosity/mass of the stellar component is not the only global property of the local ETGs that correlates with the central BH mass. In fact, the relation between the mass of the central BH and the stellar velocity dispersion $\sigma_{\star}$ or, more recently, the velocity dispersion of the globular cluster system, has been studied and it appears to be somewhat less dispersed than that with the stellar luminosity/mass (Ferrarese \& Merritt 2000; Gebhardt et al. 2000b; Tremaine et al. 2002; Gültekin et al. 2009; McConnell et al. 2011; Graham et al. 2011; Beifiori et al. 2012; Sadoun \& Colin 2012; McConnell \& Ma 2013; Pota et al. 2013; Kormendy \& Ho 2013). This relationship is possibly imprinted by the

\footnotetext{
${ }^{1}$ SISSA, Via Bonomea 265, 34136 Trieste, Italy

2 Dip. Fisica, Univ. 'Tor Vergata', Via Ricerca Scientifica 1, 00133 Roma, Italy

3 Dept. of Astron. and Inst. of Theor. Physics and Astrophysics, Xiamen Univ., 361005 Xiamen, China

${ }^{4}$ INAF-Osservatorio Astronomico di Padova, Vicolo dell'Osservatorio 5, 35122 Padova, Italy

${ }^{5}$ INAF-Osservatorio Astronomico di Brera, via E. Bianchi 46, 23807 Merate, Italy

${ }^{6}$ INFN-Sezione di Trieste, via Valerio 2, 34127, Trieste, Italy
} 
QSO feedback (but also stellar feedback can contribute); such an idea has been proposed by Silk \& Rees (1998) based on an energy argument and by Fabian (1999) based on a momentum one, and further developed by King (2003, 2005) and by Murray et al. (2005).

Also correlations between the central BH mass and the light profiles of the host galaxies have been explored (Graham et al. 2001; Lauer et al. 2007; Graham \& Driver 2007; Kormendy \& Bender 2009); these mirror the complexity of the phenomena related to the coupled evolution of the host galaxy and its central active nucleus. Comprehensive discussions of the subject have been presented by Beifiori et al. (2012) and Kormendy \& Ho (2013).

The high degree of correlation, though with significant dispersion, between the local values of $M_{\mathrm{BH}}$ and $M_{\star}$, or of $M_{\mathrm{BH}}$ and $\sigma_{\star}$, strongly suggests that the star formation on galactic scales, the accreted mass onto the central $\mathrm{BH}$ and the ensuing radiated power from the AGN have to be in a way coordinated. However, this is a reductive conclusion, since star formation, gas accretion and radiative feedback are quite complex physical processes occurring on very different spatial and temporal scales. Each of these phenomena is per se a challenging problem to treat.

While some hints on the $\mathrm{BH} /$ galaxy coevolution can be derived by focusing onto their low- $z$ properties (e.g., Kormendy \& Ho 2013), in this paper we aim at showing that the wide samples of AGNs and host galaxies observed at high redshift $(z \gtrsim 1.5)$ already yield important information on how and when the formation of the oldest stellar population in ETGs/bulges and the BH growth influence each other. As a matter of fact, both the nuclear activity (Granato et al. 2001; Richards et al. 2006; Driver et al. 2013; Kulier et al. 2013) and the formation of the old stellar populations in ETGs peak at $z \approx 2$ (e.g., Fardal et al. 2007; Driver et al. 2013).

We leave aside the second stage of star formation in galactic thin discs (see Cook et al. 2009; Driver et al. 2013) as well as the low redshift fading activity of AGNs, since there is practically no correlation between the disc luminosity/mass and the BH mass (e.g., Kormendy \& Bender 2013; Kormendy \& Ho 2013). Correspondingly, low redshift AGNs exhibit in general small Eddington ratios (e.g., Vestergaard 2004; Vestergaard \& Osmer 2009; Kelly \& Shen 2013), with the possible exception of the Narrow Line Seyfert 1 galaxies. A likely interpretation of this fading AGN phase is that it is mainly due to short episodes of accretion onto a pre-existing supermassive BH (Cavaliere \& Vittorini 2000; Ballo et al. 2007; Simmons et al. 2011; Shankar 2009; Shankar et al. 2013).

From a statistical point of view, the coevolution of the host galaxies and their supermassive BHs could be reconstructed if the stellar mass/star formation rate (SFR) distribution for the hosts and the $\mathrm{BH}$ mass/accretion rate distribution for the AGNs/QSOs at different redshifts were available. These pieces of information would provide insights at least on space- and time-averaged quantities. Progress in this respect has been tremendous in the last decade and nowadays we have sound estimates even at substantial redshift of:

- luminosity/stellar mass functions (e.g., Stark et al. 2009; Marchesini et al. 2009, 2010; Cirasuolo et al. 2010; Ilbert et al. 2013);

- far infrared (FIR) luminosity/SFR function in massive galaxies (e.g., Eales et al. 2010; Gruppioni et al. 2010, 2013; Lapi et al. 2011);

- BH masses (see Shen 2013 for a comprehensive review) and Eddington ratios (see Kelly \& Shen 2013 and references therein);

- luminosity functions of AGNs/QSOs in the X-ray (e.g., Ueda et al. 2003; Aird et al. 2008, 2010; Fiore et al. 2012a) and in the optical bands (e.g., Pei 1995; Wolf et al. 2003; Hunt et al. 2004; Richards et al. 2005, 2006; Fan et al. 2006; Fontanot et al. 2007; Croom et al. 2009; Jiang et al. 2009; Willott et al. 2010; Masters et al. 2012; Ross et al. 2013).

On top of that, recently the coevolution has been also explored by searching for nuclear activity in starforming galaxies and, viceversa, searching for star formation in AGNs/QSOs. More in detail, large statistics have been recently obtained on:

- nuclear activity, by exploiting the follow-up in X rays of galaxies with large SFR mainly selected at FIR/sub-mm wavelengths or in the $K$-band (e.g., Borys et al. 2005; Alexander et al. 2005, 2008; Laird et al. 2010; Symeonidis et al. 2010; Xue et al. 2010; Georgantopoulos et al. 2011; Carrera et al. 2011; Melbourne et al. 2011; Rafferty et al. 2011; Mullaney et al. 2012a; Johnson et al. 2013; Wang et al. 2013) and of galaxies starforming at a lower rate hence more easily selected at UV wavelengths via the stacking technique (e.g., Fiore et al. 2012b; Treister et al. 2011; Willott 2011; Basu-Zych et al. 2013);

- the star formation in AGN host galaxies, by exploiting the follow-up at FIR and (sub-)mm wavelengths of X-ray selected AGNs (e.g., Page et al. 2004, 2012; Stevens et al. 2005; Lutz et al. 2010; Shao et al. 2010; Mainieri et al. 2011; Harrison et al. 2012; Mullaney et al. 2012b; Rosario et al. 2012; Rovilos et al. 2012; Santini et al. 2012) and of optically selected QSOs (Omont et al. 1996, 2001, 2003; Carilli et al. 2001; Priddey et al. 2003; Wang et al. 2008; Walter et al. 2009; Serjeant et al. 2010; Bonfield et al. 2011; Mor et al. 2012).

These two complementary blocks of observations are of paramount relevance in determining the way stellar and BH mass grew at early times in the ETG progenitors. In the present paper we will exploit a basic model to provide a definite descriptions of the SFR and AGN lightcurves in terms of a few physical parameters. We will show that 
the comparison with the current data can constrain the model, and thus clarify the main aspects of the galaxy/AGN coevolution process. We will also point out that additional observations in X-ray, optical and FIR bands are strongly required in order to test the overall picture in detail.

The paper is organized as follows. In $\S 2$ we present a simple prescription for the time-averaged evolution of the SFR and $\mathrm{BH}$ accretion rate, following the guidance of a basic physical model, and show that it fits the statistics of the SFR (i.e., the FIR luminosity function) and the stellar mass function at high redshift. $\S 3$ is devoted to show how powerful are the data obtained by X-ray follow-up of FIR selected starforming galaxies and by FIR follow-up of X-ray and optically selected AGNs/QSOs in constraining the model. The comparison of our results with the AGN/QSO luminosity functions in the X-ray and optical bands is also presented. In $\S 4$ we show that additional observations, somewhat less systematic but of great relevance such as the BH to stellar mass ratio in optically selected QSOs, the relative abundance of obscured to unobscured AGNs/QSOs, and the measured QSO outflow rates, compare well with the predictions of the model. In $\S 5$ we discuss the prospects for direct detection of the large gas reservoir around the supermassive BHs predicted by the model. We also discuss our understanding of the gas path from the interstellar medium to the accretion disc around the supermassive BHs. In $\S 6$ we summarize our findings.

Throughout this work we adopt the concordance cosmology (see Planck Collaboration 2013), i.e., a flat universe with matter density parameter $\Omega_{M}=0.31$, Hubble constant $H_{0}=100 h \mathrm{~km} \mathrm{~s}^{-1} \mathrm{Mpc}^{-1}$ with $h=0.67$, and mass variance $\sigma_{8}=0.82$ on a scale of $8 h^{-1} \mathrm{Mpc}$. Stellar masses and FIR luminosities (or conversely SFRs) of galaxies are evaluated assuming the Chabrier's (2003) initial mass function (IMF) and the spectral energy distribution (SED) of SMM J2135-0102, a typical high-redshift starbursting galaxy (very similar to the local ULIRG Arp220; see Lapi et al. 2011 for details). Specifically, the luminosity associated to the SFR reads $3.2 \times 10^{43}\left(\dot{M}_{\star} / M_{\odot} \mathrm{yr}^{-1}\right) \mathrm{erg} \mathrm{s}^{-1}$, which practically coincides with the FIR luminosity $L_{\mathrm{FIR}}$ if dust absorbs almost all of the stellar emission. By FIR we consider the restframe wavelength range from $\lambda \approx 40 \mu \mathrm{m}$ to $\lambda \approx 500 \mu \mathrm{m}$. In general, we can assume that this spectral region is dominated by the emission of dust associated with star formation, although a contribution from a torus around an AGN may be not completely negligible (e.g., Granato \& Danese 1994; Leipski et al. 2013). As to the nuclear emission, we will indicate with $L_{\mathrm{AGN}}$ the bolometric output, and with $L_{B}=L_{\mathrm{AGN}} / k_{B}, L_{X}=L_{\mathrm{AGN}} / k_{X}$ the powers in the optical and in the $2-10 \mathrm{keV}$ X-ray bands, adopting the bolometric corrections $k_{B}$ and $k_{X}$ by Hopkins et al. (2007; see also Marconi et al. 2004, Vasudevan \& Fabian 2007). The values depend on the AGN luminosity (or on the Eddington ratio), and span the ranges $k_{B} \sim 8-15$ and $k_{X} \sim 15-100$ for $L_{\mathrm{AGN}} \sim 10^{43}-10^{47}$ erg s ${ }^{-1}$. Note that we will restrict ourselves to $L_{X} \gtrsim 10^{42} \mathrm{erg} \mathrm{s}^{-1}$, to avoid appreciable contaminations of the X-ray emission by star formation (see Symeonidis et al. 2011).

\section{A BASIC MODEL}

The observations mentioned in $\S 1$ can be exploited to cast light on how the relationships between star formation and $\mathrm{BH}$ accretion in primeval galaxies have been set up. To this purpose we need to exploit a simple but physically motivated description of the SFR and of the accretion rate onto the $\mathrm{BH}$ as a function of galactic time within dark matter halos of given mass and formation redshift. Then the SFR is converted into FIR luminosity, under the assumption that most of the star formation in massive galaxies occurs in a dusty environment. The accretion rate is converted into bolometric luminosity of the AGN by assuming a matter-to-radiation conversion efficiency, and eventually into luminosity at specific wavelengths through bolometric corrections. The light curves of the host galaxy and of the AGN are then exploited for the computation of various statistics to be compared with the data.

We take as a guidance the framework originally proposed in Granato et al. (2004), which has been successful in reproducing the statistics of galaxies selected at $850 \mu \mathrm{m}$, of passively evolving galaxies, and of AGNs/QSOs at substantial redshift. Lapi et al. (2006, 2011) and Cai et al. (2013) have further developed the original formulation of the model, and showed that it fits the galaxy number counts at (sub-)mm wavelengths (e.g., Clements et al. 2010; Vieira et al. 2010, 2013) and the luminosity functions of sub-mm selected galaxies (Eales et al. 2010; Lapi et al. 2011; Gruppioni et al. 2013). Furthermore, the model has been used to estimate the number of sub-mm selected gravitationally lensed galaxies (Perrotta et al. 2003; Negrello et al. 2007; Lapi et al. 2012), a prediction fully confirmed by observations (Negrello et al. 2010; Gonzalez-Nuevo et al. 2012; Weiss et al. 2013). Even the correlation function of both QSOs and sub-mm selected galaxies are very well reproduced, ensuring that the model correctly locate galaxies and QSOs into massive dark matter halos (Xia et al. 2012).

The model is based on a few assumptions, that we recall next. Concerning the formation of the dark matter hosts, it exploits the outcomes of many intensive $N$-body simulations and semianalytic studies (e.g., Zhao et al. 2003; Wang et al. 2011; Lapi \& Cavaliere 2011). These have recognized that massive halos undergo an early phase of fast collapse, during which the central regions reach rapidly a dynamical quasi-equilibrium; a subsequent slower accretion phase mainly affects the halo outskirts. The transition between the two phases can be identified with the formation redshift. The halo formation rates at redshift $z \gtrsim 1.5$ and for halo masses $M_{\mathrm{H}} \gtrsim 10^{10} M_{\odot}$ derived from cosmological $N$-body simulations are very well approximated by the analytical formulae given in Lapi et al. (2013), which have been exploited in the present work.

The mass of baryons associated to a dark halo is about $20 \%$ of the total one. It has been shown that in the local Universe the largest ratio of stellar-to-halo mass amounts to $M_{\star} / M_{\mathrm{H}} \lesssim 0.03$ in galactic halos of mass $M_{\mathrm{H}} \approx 10^{12} M_{\odot}$ (e.g., Vale \& Ostriker 2004; Shankar et al. 2006; Guo et al. 2010; Moster et al. 2010, 2013). Only $\lesssim 15 \%$ of the available baryons are finally locked up into stars and such a percentage can be $\lesssim 10 \%$ at higher redshift (Moster et al. 2013). Assuming a Chabrier (2003) IMF the fraction of baryons passed through the stellar cycle at high redshift 
is $\lesssim 20 \%$. Thus very likely $\lesssim 40 \%$ of the baryons associated to dark halos are possibly involved in the formation of galaxies. This corresponds to the mass which is involved in the early fast collapse. It is worth noticing that the average inefficiency of star formation is highlighted by the small ratio of density parameters in stars and baryons $\Omega_{\star} / \Omega_{b} \approx 5 \%$ (e.g., Fukugita \& Peebles 2004; Shankar et al. 2006; Li \& White 2009), corresponding to $\Omega_{\star} / \Omega_{\mathrm{DM}} \approx 1 \%$ (e.g., Moustakas et al. 2013).

The fast collapse is expected to be inhomogeneous and to proceed through generation of clumps (see Ceverino et al. 2010; Bournaud et al. 2014). Gravitational instabilities, tidal torques and dynamical friction among the starforming clumps, coupled with large gas flows generated by stellar winds and supernova explosions, strongly favor the dissipation of the angular momentum left over by the dynamical relaxation occurred during the fast collapse (see Noguchi 1999; Immeli et al. 2004; Elmegreen et al. 2008). In this framework, our model treats the coevolution of the galaxy and its central $\mathrm{BH}$ by assuming that the angular momentum of the baryonic clumps is dissipated by such mechanisms on a timescale comparable with or shorter than the duration $\Delta t_{\text {burst }}$ of the starbursting activity. Therefore the evolution of massive galaxies is dictated by secular internal processes occurring in the central regions of galactic halos. Since these processes start, major mergers are no longer relevant for the galaxy/QSO evolution and are neglected. This is a crucial feature of our model, as opposed to other treatments (for a list, see Scannapieco et al. 2012; for a critical view, see Frenk \& White 2012 and Kaviraj et al. 2013).

The galaxy is considered to be constituted by two gas phases, the infalling hot/warm gas with mass $M_{\text {inf }}$ and the cold gas with mass $M_{\text {cold }}$, and by stars (forming and dying). The hot/warm gas condenses into the cold one at a rate $\dot{M}_{\text {cold }}=M_{\text {inf }} / t_{\text {cond }}$, where the timescale $t_{\text {cond }}$ is the longer between the free fall and the cooling timescales, computed at the virial radius by assuming for the hot/warm gas a clumping factor $\approx 7$ (see Lapi et al. 2006); an analytical approximation reads

$$
t_{\text {cond }} \approx 7 \times 10^{8}\left(\frac{1+z}{3.5}\right)^{-1.5}\left(\frac{M_{\mathrm{H}}}{10^{12} M_{\odot}}\right)^{0.2} \mathrm{yr} .
$$

The average SFR is computed as $\dot{M}_{\star}=M_{\text {cold }} / t_{\text {cond }} \times s$, and is assumed to occur on a timescale shorter than the condensation time since the cold gas is expected to fragment and to increase its clumping factor well above that of the hot/warm component. The factor $s$ is adjusted at a value around 5 to fit the aforementioned wide sets of data, and in particular the sub-mm luminosity functions and counts.

On the other hand, the feedback from stars, proportional to the number of supernovae (SN) and hence to the SFR, removes the cold gas at a rate

$$
\dot{M}_{\mathrm{cold}}^{\mathrm{SN}}=\frac{N_{\mathrm{SN}} \epsilon_{\mathrm{SN}} E_{\mathrm{SN}}}{E_{\mathrm{bind}}} \dot{M}_{\star},
$$

where $N_{\mathrm{SN}} \approx 1.4 \times 10^{-2} / M_{\odot}$ is the number of SNae per unit solar mass condensed into stars, $E_{\mathrm{SN}} \approx 10^{51} \mathrm{erg}$ is the kinetic energy released per SN explosion, $E_{\text {bind }} \approx 3 \times 10^{14}\left(M_{\mathrm{H}} / 10^{12} M_{\odot}\right)^{2 / 3}[(1+z) / 3.5] \mathrm{cm}^{2} \mathrm{~s}^{-2}$ is the specific binding energy of the gas in the halo; $\epsilon_{\mathrm{SN}}=0.05$ is the standard value adopted for the SN feedback efficiency (e.g., White \& Frenk 1991; Cole et al. 2000).

Granato et al. (2004) were among the first to work out a quantitative model for the feedback from nuclear activity. According to this model, the feedback is able to affect the most external parts of the galaxy and to act on the hot/warm and cold gas in a way proportional to their fractional mass. For the cold gas the rate of removal is

$$
\dot{M}_{\text {cold }}^{\mathrm{QSO}}=f_{\text {cold }} \frac{L_{\mathrm{QSO}}}{E_{\mathrm{bind}}}
$$

where $f_{\text {cold }}$ is the cold to total gas mass fraction, and $L_{\mathrm{QSO}} \approx 2 \times 10^{44} \epsilon_{\mathrm{QSO}}\left(M_{\mathrm{BH}} / 10^{8} M_{\odot}\right)^{3 / 2} \mathrm{erg} \mathrm{s}^{-1}$ is the mechanical energy delivered from the QSO in terms of a strength parameter $\epsilon_{Q S O}$; values $\epsilon_{Q S O} \sim 1$ are required to reproduce the bright end of the stellar mass function and of the QSO luminosity functions (see Lapi et al. 2006 and Cai et al. 2013 for details). A similar expression holds for the feedback on the hot/warm component.

Examples of the evolution with galactic age of the FIR luminosity associated to the SFR are illustrated in Fig. 1. The curves refer to halo masses $M_{\mathrm{H}}=2$ and $6 \times 10^{12} M_{\odot}$ at $z=2$. For these massive galaxies, the SFR can be approximated for most of the time ( $\gtrsim 80 \%$ of the burst duration) by a constant with a dependence on halo mass and redshift given by

$$
\dot{M}_{\star}=70\left(\frac{M_{\mathrm{H}}}{10^{12} M_{\odot}}\right)\left(\frac{1+z}{3.5}\right)^{2.1} M_{\odot} \mathrm{yr}^{-1} .
$$

The duration of the main burst is approximately $\Delta t_{\text {burst }} \approx 7 \times 10^{8}[(1+z) / 3.5]^{-1.5}$ yr.

In the model the approximate constancy of the SFR mirrors that of the mass in cold gas. In turn, the latter stems from the balance among: (i) the condensation rate of warm/hot gas into the cold phase; (ii) the rate of star formation; (iii) the rate of mass restitution from stellar evolution to the cold gas phase; (iv) the rate of cold gas removal by stellar feedback (SN and stellar winds). We stress that such a balance also ensures a rapid increase of the metallicity in the cold gas and hence in the formed stars. A rapid chemical enrichment is fundamental in creating conditions apt to a rapid formation of large amounts of dust in massive galaxies at high redshift; in combination with the relatively short duration $\lesssim 0.5-1$ Gyr of the star formation the enrichment is also relevant to produce chemical abundances and 
$\alpha$-enhancement in agreement with those observed for local massive ETGs (see Silva et al. 1998; Granato et al. 2004; Lapi et al. 2006, 2011).

The FIR lightcurves depend on halo mass and redshift; to derive the luminosity function of starforming galaxies in the FIR the galaxy luminosity must be convolved with the halo formation rate (see Appendix). The outcome is illustrated in Fig. 2, which shows that the model nicely reproduces the observed luminosity functions of starforming galaxies at high redshift $z \gtrsim 1.5$. We stress that also galaxy counts, clustering properties and the cosmic infrared background autocorrelation function at (sub-)mm wavelengths can be reproduced by exploiting a quite standard spectral energy distribution for dust emission, similar to that of local ULIRGs (see Lapi et al. 2011; Cai et al. 2013).

The stellar mass is linearly increasing with time for $t \lesssim \Delta t_{\text {burst }}$ and can be approximated by

$$
M_{\star}(t) \approx 5 \times 10^{10}\left(\frac{t}{\Delta t_{\text {burst }}}\right)\left(\frac{M_{\mathrm{H}}}{10^{12} M_{\odot}}\right)\left(\frac{1+z}{3.5}\right)^{0.6} M_{\odot}
$$

if a Chabrier (2003) mass function is assumed; due to gas recycling, the present-day mass in stars is about half of the mass in stars formed before $\Delta t_{\text {burst }}$. The evolution of the stellar mass is presented in Fig. 3, while the stellar mass function at the relevant redshifts yielded by the model is confronted with observations in Fig. 4 . The agreement corroborates the physical assumptions of the model. Its effectiveness resides in the capability of locating the quite large observed SFR at high redshift in the most massive, strongly clustered halos.

As for the nuclear activity, the gas flowing toward the $\mathrm{BH}$ through the accretion disc produces a time-averaged radiative output

$$
\epsilon c^{2} \dot{M}_{\mathrm{accr}}=L_{\mathrm{AGN}}=\lambda L_{\mathrm{Edd}}
$$

where $\dot{M}_{\text {accr }}$ is the accretion rate, $0.06 \lesssim \epsilon \lesssim 0.3$ is the mass to radiation conversion efficiency $(\epsilon=0.15$ has been assumed in this work, see the discussion in $\S 5.2 .3)$, and $\lambda L_{\text {Edd }}$ is the luminosity in terms of the Eddington one. Lapi et al. (2006) have shown that the Eddington ratio has on average to increase with redshift (for $z \gtrsim 1.5$ ) as $\lambda(z)=-1.15+0.75(1+z)$ in order to reproduce the QSO luminosity functions for $1.5 \lesssim z \lesssim 6$. The maximum allowed value $\lambda \lesssim 4$ is in good agreement with recent estimates from observations (see Kelly \& Shen 2013). If $\epsilon$ and $\lambda$ are assumed to stay on average constant during most of the accretion time (we defer to $\S 5.2 .3$ the discussion of these assumptions), Eq. (6) implies that the BH mass and luminosity grow exponentially on a timescale $\tau_{\text {ef }}=\epsilon / \lambda(1-\epsilon) \times t_{\text {Edd }}$ with $t_{\mathrm{Edd}} \approx 4.5 \times 10^{8} \mathrm{yr}$; we recall that $\dot{M}_{\mathrm{BH}}=(1-\epsilon) \dot{M}_{\mathrm{accr}}$.

The mean observed ratio between the stellar and BH mass in local massive ETGs and bulges is $\Gamma_{0}=M_{\mathrm{BH}} / M_{\star} \approx$ $3 \times 10^{-3}$ though with a large scatter of $\approx 0.4$ dex (e.g., Magorrian et al. 1998; Gebhardt et al. 2000a; Marconi \& Hunt 2003; Häring \& Rix 2004; Ferrarese \& Ford 2005; Bennert et al. 2011; Graham et al. 2011; Beifiori et al. 2012; McConnell \& Ma 2013; Kormendy \& Ho 2013). This suggests that the cold gas inflows from galactic scales to the central regions $(\lesssim 100 \mathrm{pc})$ at a rate proportional to the $\mathrm{SFR}$, i.e.,

$$
\dot{M}_{\text {inflow }}=\alpha_{\text {res }} \times 10^{-3} \dot{M}_{\star} \text {. }
$$

The efficiency $\alpha_{\text {res }}\left(M_{\mathrm{H}}, z\right)$ is possibly a function of halo mass and formation redshift, and depends on the particular process considered for the gas to lose its angular momentum on large scales (see Granato et al. 2004; Lapi et al. 2006). The above proportionality is also supported, though with a substantial scatter, by several numerical simulations as recalled in $\S 5.2$. In particular, Hopkins \& Quataert $(2010,2011)$ show that gravitational instabilities can trigger star formation and at the same time funnel part of the interstellar medium (ISM) toward the central region ( $\lesssim 100$ pc), where the gas attains large surface densities.

At this stage the gas inflowing from the ISM can be either directly accreted onto the BH or can pile up into a reservoir. As we shall see in $\S 3$, the data do not support a time-averaged proportionality between the bolometric AGN luminosity and the FIR emission associated with the SFR of the host galaxy.

In addition, if one assume a conceivably small seed $\mathrm{BH}$ mass $M_{\mathrm{BH}} \sim 10^{2}-10^{3} M_{\odot}$ (for a review, see Volonteri 2010), the large and constant SFR implies the rate

$$
\dot{M}_{\text {res }}=\dot{M}_{\text {inflow }}-\dot{M}_{\text {accr }}=\alpha_{\text {res }} \times 10^{-3} \dot{M}_{\star}-\frac{\lambda}{\epsilon} \frac{L_{\text {Edd }}}{c^{2}},
$$

to be positive for many $e$-folding times, allowing a reservoir to form and its mass to significantly increase; e.g., for a $\dot{M}_{\star} \gtrsim 10^{2} M_{\odot} \mathrm{yr}^{-1}$ and a seed BH mass $M_{\bullet} \approx 10^{3} M_{\odot}$, one has the formation of a reservoir $\left(\dot{M}_{\text {res }} \gtrsim 0\right)$ unless $\lambda / \epsilon \gtrsim 10^{5}$ is exceptionally high (cf. discussion in $\S 5.2 .3$ ). Examples of the time evolution for the reservoir and $\mathrm{BH}$ masses are plotted in Fig. 3. The reservoir can be related to the so called torus, observed in nearby AGNs and called for to explain the AGN phenomenology (e.g., Antonucci 1993; Granato \& Danese 1994; Urry \& Padovani 1995). Observations, at least of low redshift objects (see, e.g., Davies et al. 2007; Muller-Sanchez et al. 2009 ; Krips et al. 2011; Diamond-Stanic \& Rieke 2012; Sani et al. 2012; Storchi-Bregmann et al. 2012; Hönig et al. 2013), have revealed the presence of such reservoirs, with sizes from a few to several tens of parsecs; the reservoirs have been found to be rich in molecular gas and dust, and often accompanied by localized star formation.

Additional physical mechanisms, such as turbulence generated by SN explosion, gravitational tidal torques, dynamical friction among clumps, can further reduce the angular momentum of the gas making it ready to flow through the accretion disc (see $\S 5.2$ for a discussion). The gas piled up in the reservoir is large enough to sustain accretion 
during the two final $e$-folding times, when the QSO reaches its maximum luminosity and the $\mathrm{BH}$ acquires most of its final mass. During this short epoch lasting $\lesssim 10^{8}$ yr powerful winds can be generated, capable of removing most of the gas within the host galaxy, of quenching the star formation, and thus stopping the fueling of the reservoir. The gas remaining in the reservoir still flows toward the $\mathrm{BH}$, though at a decreasing rate; thus the accretion luminosity decreases to only a fraction of the Eddington limit.

If, and only if, a significant fraction of the gas stored in the reservoir is finally accreted onto the seed $\mathrm{BH}$, then the correlation between the mass in old stars and the BH mass found in local ETGs is automatically set up. For a Chabrier IMF only about half (in detail, a factor 1/1.7) of the mass in stars formed early is presently still there; this implies that $M_{\mathrm{BH}} \approx 1.7 \times \alpha_{\mathrm{res}} \times 10^{-3} M_{\star}$ holds, so average values $\alpha_{\mathrm{res}} \sim 2$ would yield the local ratio $\Gamma_{0}$. By the same token, $\alpha_{\text {res }}$ should exhibit the same scatter of about 0.4 dex observed in $\Gamma_{0}$ (e.g., Beifiori et al. 2012; McConnell \& Ma 2013; Kormendy \& Ho 2013). This conclusion is quite reasonable, since $\alpha_{\text {res }}$ embodies a large number of physical mechanisms and astrophysical settings, as we shall discuss in $\S 5.2$. Actually this scatter is essential to reproduce the QSO luminosity functions at very high redshift $(z \gtrsim 4)$, that sample only $\gtrsim 3 \sigma$ objects (Wyithe \& Loeb 2003; Mahmood et al. 2005; Lapi et al. 2006; Fanidakis et al. 2012). All in all, the final mass of the BH itself can be approximated as (see Fig. 3)

$$
M_{\mathrm{BH}} \approx 5 \times 10^{7} \alpha_{\mathrm{res}}\left(\frac{M_{\mathrm{H}}}{10^{12} M_{\odot}}\right)\left(\frac{1+z}{3.5}\right)^{0.6} M_{\odot} .
$$

In the original formulation by Granato et al. (2004) the star formation and disc accretion are abruptly shut off when the mechanical energy delivered by the active nucleus is large enough to unbind the gas from the galaxy. From Eqs. (2) and (3) we can infer that this occurs at a galactic age $t_{\mathrm{QSO}}$ when the energy feedback from the QSO overwhelms that from SNae; such a time is found to be very close to $\Delta t_{\text {burst }}$ as defined below Eq. (4). Instead, in the present work we allow for a more educated decline, that we will show to be essential in reproducing the observational data (see $\S 3.2$ ). As a matter of fact, the feedback can require some time in order to become fully effective. For instance, King (2010) has shown that the expansion of a momentum-driven shell can reach $20 \mathrm{kpc}$ in a massive galaxy on a timescale of about $6 \times 10^{7} \mathrm{yr}$, similar to the typical $e$-folding time $\tau_{\mathrm{ef}} \approx 4 \times 10^{7} \mathrm{yr}$. Thus in the model we assume that at the galactic time $t_{\mathrm{decl}}=t_{\mathrm{QSO}}+n \tau_{\mathrm{ef}}$ with $n \approx 2$ both the ISM and the reservoir are affected by the QSO feedback.

For simplicity, we assume that the SFR fades on a timescale $\tau_{\mathrm{SFR}}$ and the accretion rate decreases on a timescale $\tau_{\mathrm{AGN}}$, free parameters to be set by comparison with the data in $\S 3$. We shall express them in terms of the timescale $\tau_{\text {ef }}$ for $\mathrm{BH}$ growth. The AGN declining phase lasts until the gas mass piled up in the reservoir is exhausted. On the other hand, it does not occur if the gas in the reservoir is exhausted in the time interval between $t_{\mathrm{QSO}}$ and $t_{\text {decl }}$. Presence or absence of the AGN declining phase depends on the parameter $\alpha_{\text {res }}$ following Eq. (7), i.e., on the efficiency of the gas storing within the reservoir; the transition between these two regimes occurs around $\alpha_{\text {res }} \approx 5$. Examples of the AGN light-curves are plotted in Fig. 1.

\section{THE RELATIONSHIP BETWEEN STAR FORMATION AND BH GROWTH: DATA AND MODEL COMPARISON}

The coevolution of AGNs and of their host galaxies at high redshift is mainly traced by observations aimed at estimating the relationship between the star formation in the host and the nuclear activity due to accretion onto the central BH. There are two main ways to proceed. In the first, galaxies are selected on the basis of their SFR and followed up with X-ray observations looking for nuclear activity. In the second, the AGN is selected in X-rays and then followed up with observations looking for star formation in the host. The main outcomes of these observations are statistics on detections and on properties such as stellar mass in the host and BH mass. These statistics depend on the limiting flux/luminosity of the selection and of the ensuing follow-up. Details on model estimates are given in the Appendix.

\subsection{The relationship between SFR and AGN luminosity in high redshift starbursting galaxies}

We begin with discussing X-ray observations of high redshift galaxies mainly selected on the basis of their SFR. The purpose is to illustrate how powerful are accurate statistics derived from FIR and X-ray observations in constraining the evolution of the star formation in the host and of the BH growth, using the model lightcurves presented above as a guidance. The available statistics already strongly support the conclusion that in an early phase the AGN luminosity is increasing exponentially, while the SFR is almost constant.

\subsubsection{FIR and (sub-)mm galaxies in X-rays: observations vs. model predictions}

In Fig. 5 we present the fraction of AGNs detected in X-rays $(2-10 \mathrm{keV}$ band) as a function of the host FIR luminosity and of the X-ray detection threshold. We warn the reader that for different data samples the minimum $\mathrm{X}$-ray luminosity for detection is somewhat uncertain (depending on flux limit, absorption correction, redshift, and so on). The model agrees with the data in predicting an increasing detection fraction with increasing SFR (or $L_{\text {FIR }}$ ) at given X-ray detection threshold $L_{X}$.

In the model the FIR luminosity is mainly a function of the halo mass (see Eq. 4 ) and changes by a factor of $\lesssim 3$ for most $\gtrsim 80 \%$ of the star formation duration (see Fig. 1). On the other hand, the accretion luminosity is increasing exponentially on a timescale $\tau_{\text {ef }}$. As a consequence, the time lapse during which the central AGN is brighter than the X-ray detection threshold is only a fraction of the starburst duration. The increasing fraction of detected AGNs with increasing SFR of the host galaxy reflects the higher power that can be attained by more massive BHs in more 
massive galaxy halos (see Eq. 9). Plainly, at given halo mass, the higher is the X-ray detection threshold, the shorter is the fraction of time spent by the AGN above that limit. The model also predicts a slight redshift dependence, due to the combined effect of the increase in Eddington ratio $\lambda$ (implying a decrease of $\tau_{\text {ef }}$ ) and of the decrease in the star formation duration with increasing redshift.

Conversely, a shorter or a longer timescale for the evolution of the accretion luminosity would imply detection rates higher or lower than observed, respectively. For instance, in the hypothesis that the luminous accretion rate during the star formation epoch is proportional to the large-scale SFR (e.g., Mullaney et al. 2012a; Silk 2013), $\dot{M}_{\mathrm{accr}} \approx \Gamma_{0} \dot{M}_{\star}$ with $\Gamma_{0} \approx 3 \times 10^{-3}$, we get $L_{\mathrm{FIR}} / L_{\mathrm{AGN}} \approx 1(\epsilon / 0.15)^{-1}$. Then the expected X-ray luminosity would exceed $L_{X} \approx 10^{42}\left(k_{X} / 25\right)^{-1}\left(\Gamma_{0} / 3 \times 10^{-3}\right)\left(\dot{M}_{\star} / M_{\odot} \mathrm{yr}^{-1}\right) \mathrm{erg} \mathrm{s}^{-1} \gtrsim 10^{43} \mathrm{erg} \mathrm{s}^{-1}$ whenever $\dot{M}_{\star} \gtrsim 10 M_{\odot} \mathrm{yr}^{-1}$ and $L_{\mathrm{FIR}} \gtrsim 3 \times 10^{44} \mathrm{erg} \mathrm{s}^{-1}$, adopting a standard bolometric correction (e.g., Hopkins et al. 2007). Such an AGN X-ray luminosity would imply that in galaxies with $L_{\mathrm{FIR}} \gtrsim 3 \times 10^{44} \mathrm{erg} \mathrm{s}^{-1}$ the nuclear activity is always detected, in contrast with the observations presented in Fig. 5.

To escape the limits set by observations, one has to assume that all AGNs hosted by FIR bright galaxies have extreme X-ray absorbing column densities $\left(N_{\mathrm{H}} \gg 10^{24} \mathrm{~cm}^{-2}\right)$. This would yield a selection bias which allows us to detect only the objects with lower column densities, implying a reduced detection fraction. Although quite large column densities $\left(N_{\mathrm{H}} \gtrsim 3 \times 10^{23} \mathrm{~cm}^{-2}\right.$ ) have been observed in several (sub-)mm selected galaxies, a significant fraction of objects exhibits lower column densities (see Alexander et al. 2005; Laird et al. 2010; Georgantopoulos et al. 2011; Rafferty et al. 2011); in particular, no correlation between FIR luminosity and column density has been observed.

The FIR/submm selection elicits galaxies with bolometric luminosity associated to star formation larger than the AGN bolometric luminosity $\left(L_{\mathrm{FIR}} / L_{\mathrm{AGN}} \gtrsim 1\right.$; see Alexander 2005; Georgantopoulos et al. 2011; Laird et al. 2010; Rafferty et al. 2011; Johnson et al. 2013; Wang et al. 2013). The few objects that exhibit a ratio $L_{\mathrm{FIR}} / L_{\mathrm{AGN}} \sim 1$ have a large X-ray power $L_{X} \gtrsim 3 \times 10^{44} \mathrm{erg} \mathrm{s}^{-1}$ and large luminosity in the $\mathrm{H} \alpha$ line (Alexander et al. 2008). As for the model predictions, the AGN bolometric luminosity approaches and possibly exceeds the galaxy FIR luminosity only for a couple of $e$-folding times before its maximum (see Fig. 1). In addition, to get $L_{X} \gtrsim 10^{44} \mathrm{erg} \mathrm{s}^{-1}$, the minimu X-ray luminosity often assumed to define X-ray QSOs, a value $\dot{M}_{\star} \gtrsim 100 M_{\odot} \mathrm{yr}^{-1}$ or correspondingly $L_{\mathrm{FIR}} \gtrsim 3 \times 10^{45}$ $\operatorname{erg~} \mathrm{s}^{-1}$ is required.

The claim of having detected stacked X-ray emission from Lyman Break Galaxies (LBGs) at redshift $z \gtrsim 6$ by Treister et al. (2011) has been shown to be uncorrect, being mainly due to problems in the background subtraction (Willott 2011; Fiore et al. 2012b). Basu-Zych et al. (2013) pointed out that the contribution from LBGs at $z \gtrsim 6$ to the X-ray background is only minor. As can be seen from their UV restframe luminosity function (e.g., Bouwens et al. 2011; McLure et al. 2013; Oesch et al. 2013), most LBGs at $z \gtrsim 6$ exhibit in fact $\dot{M}_{\star} \lesssim 2-5 M_{\odot} \mathrm{yr}^{-1}$. In the model (see Mao et al. 2007) these SFRs correspond to halos with $\widetilde{M_{\mathrm{H}}} \lesssim 5 \times 10^{10} M_{\odot}$, wherein the BH can only acquire a relatively small mass $M_{\mathrm{BH}} \lesssim 10^{5} M_{\odot}$.

\subsubsection{K-band selected starforming galaxies}

The sample analyzed by Mullaney et al. (2012a), primarily selected in the $K$-band (with $B z K$ color criteria chosen to ensure presence of star formation), allows us to introduce the galaxy mass in the study of the relationship between star formation and AGN activity. In the following we focus on the sample at $z \approx 2$, relevant for our work. Because of the spectral coverage from the UV to $24 \mu \mathrm{m}$ and low detection limits, the star formation can be probed down to small rates. The AGN activity is inferred from X-ray observations with $4 \mathrm{Ms}$ exposure time, thus reaching detection thresholds $L_{X} \approx 10^{42} \mathrm{erg} \mathrm{s}^{-1}$ at $z \approx 2$. Such a luminosity corresponds to central BHs of relatively low mass $M_{\mathrm{BH}} \sim 10^{5}-10^{6} M_{\odot}$.

The detected AGN fraction as a function of FIR luminosity for the sample of Mullaney et al. (2012a) is very similar to the results obtained for FIR or (sub-)mm selected galaxies (see also Fig. 5), implying that the primary $K$-band selection is not introducing a relevant bias with respect to a pure FIR selection. Moreover, the average ratio of FIR to AGN bolometric luminosity is large $\left(4 \lesssim L_{\mathrm{FIR}} / L_{\mathrm{AGN}} \lesssim 9\right)$, as found for (sub-)mm selected galaxies.

The results of Mullaney et al. (2012a) show that the AGN detection fraction is increasing with the stellar mass (see Fig. 6); the model reproduces this behavior. However, these results are not independent of those presented in Fig. 5 . This is illustrated in Fig. 7, where data show an approximately linear dependence of stellar mass on FIR luminosity or, equivalently, on SFR. In the latter figure the solid black line shows the relation $M_{\star}-L_{\text {FIR }}$ between the average final stellar mass and the FIR luminosity predicted by the model for a sample of star forming galaxies at redshift $z \approx 2$, independently of the nuclear activity; this relation can be understood on the basis of Eqs. (4) and (5). We notice that there is very weak or no dependence on the X-ray detection threshold, since the relationship is dictated only by the star formation history. The change of the $M_{\star}-L_{\text {FIR }}$ relation with the redshift, apparent in Fig. 7 stems from the different dependence of mass and SFR on $z$. This also explains why the fraction of detected AGNs at fixed stellar mass is almost redshift independent (see Fig. 6) while the fraction at fixed FIR luminosity decreases with decreasing $z$ (see Fig. 5).

Fig. 8 illustrates the predictions of the model for the ratio $\left\langle L_{X}\right\rangle /\left\langle L_{\mathrm{FIR}}\right\rangle$ between the average X-ray and FIR luminosities of FIR-selected galaxies as a function of the stellar mass. We compare the model outcome with the data by Mullaney et al. (2012a). Though the latter refer to both detected and undetected sources, the authors point out that the X-ray counts for each bin in mass are dominated $(\gtrsim 80 \%)$ by X-ray detected galaxies. For masses large enough to allow detection, the ratio keeps almost constant.

The model reproduces such a behavior despite it features in individual galaxies a SFR almost constant and a BH 
accretion rate exponentially increasing. This comes from the primary selection in the $K$-band, which biases toward late times of the evolution, where $L_{\mathrm{AGN}}$ is rapidly approaching $L_{\mathrm{FIR}}$. Specifically, from the data it is apparent that the AGN bolometric luminosity (with $k_{X} \approx 40$ for reference; see Hopkins et al. 2007) is still a factor of several below $L_{\mathrm{FIR}}$, and it will take another couple of $e$-folding time to attain it.

In Fig. 9 we show the ratio $L_{\mathrm{FIR}} / L_{\mathrm{AGN}}$, the specific SFR (SSFR) $\dot{M}_{\star} / M_{\star}$ and the specific $\mathrm{BH}$ accretion rate $\dot{M}_{\mathrm{BH}} / M_{\mathrm{BH}}$ as a function of time as predicted by the model for a starforming galaxy at redshift $z \approx 2$; we focus on a halo mass $M_{\mathrm{H}} \approx 2 \times 10^{12} M_{\odot}$, corresponding to a stellar mass of $M_{\star} \approx 6 \times 10^{10} M_{\odot}$, typical of the sample considered by Mullaney et al. (2012a). The range of the observed values of the sSFR and of the luminosity ratio are marked on the respective model predictions. It is apparent that the data on the luminosity ratio single out a galaxy age $\approx 9-11 \tau_{\text {ef }}$, corresponding to $70-80 \%$ of the star formation duration. In terms of sSFR the time spanned by the data is larger, possibly due to the larger uncertainties in the mass estimates.

Therefore, the selection of Mullaney et al. (2012a) picks out objects that are on the average detected about $2-3$ $e$-folding times before the peak of the AGN activity; as a consequence, they should exhibit a BH to stellar mass ratio lower than the local one by a factor $5-10$. This is close to the estimate obtained by Alexander et al. (2008) for a sample of (sub-)mm selected galaxies exhibiting nuclear activity. Relatedly, in the model the specific BH accretion rate stays constant at values around $10 \mathrm{Gyr}^{-1}$, mirroring the exponential time behavior of the $\mathrm{BH}$ accretion.

In conclusion, the data of Mullaney et al. (2012a) concur with the FIR-selected samples in supporting the view that the $\mathrm{BH}$ and stellar mass are assembling in parallel, but on different timescales. While the stellar mass increases almost linearly with galactic age for a period $\Delta t_{\text {burst }} \approx 7 \times 10^{8}$ yr at $z \approx 2.5$, the BH mass is increasing exponentially on a timescale $\sim \Delta t_{\text {burst }} / 15$. Such a timescale indicates that during this epoch the BH accretion is in a self-regulated regime. In $\S 4.1$ we shall see that this conclusion is also supported by the estimates of the central BH mass in FIR and (sub-)mm selected galaxies. More detailed statistics on the nuclear activity in FIR selected galaxies would allow us to determine with high precision the ratio between the duration of the star formation and the $e$-folding timescale of the $\mathrm{BH}$ accretion.

\subsection{Star formation in high redshift AGNs: the quenching of the star formation and of the nuclear activity}

The data discussed above allowed us to reconstruct the growth phase of the BH mass and of the nuclear activity, when star formation in the host galaxy is still significant. We recall that such epoch is characterized by objects with $L_{\mathrm{FIR}} / L_{\mathrm{AGN}} \gtrsim 1$.

Here we show that determinations of the SFR in high- $z$, X-ray or optically selected AGNs, allow us to explore the phases when the AGN/QSO approaches its maximum mass/luminosity and the quenching of star formation and of nuclear activity sets in. Our analysis focuses on what can be learnt on these quenching timescales from (i) FIR and (sub-)mm observations of X-ray selected AGNs and QSOs; (ii) star formation in optically selected QSOs.

\subsubsection{Star formation in high redshift X-ray selected AGNs and QSOs}

In Fig. 10 we present the results relative to the statistics of the X-ray selected AGNs whose host galaxy has been detected in the FIR/(sub-)mm. Although some caution must be used in interpreting data obtained with different luminosity threshold in the FIR, nevertheless it is apparent that only a quite small fraction of powerful X-ray AGNs with $L_{X} \gtrsim 10^{44} \mathrm{erg} \mathrm{s}^{-1}$ are detected in the FIR. Such a small fraction suggests that the most luminous AGNs extend their X-ray emission beyond the epoch of constant and large SFR into a phase when the star formation has been quenched more rapidly than the nuclear activity.

In the model this later phase is present only if the reservoir is not exhausted before the feedback quenches the star formation; in such cases, the accretion can continue for some additional time. The mass in the reservoir is determined by the efficiency of the mechanisms capable of transfering the angular momentum of the cold gas involved in the star formation process; in the model this aspect is encapsulated into the parameter $\alpha_{\text {res }}$ in Eq. (5). On the other hand, the distribution of $\alpha_{\text {res }}$ reflects into the distribution of the $\mathrm{BH}$ to stellar mass ratio $\Gamma_{0}=M_{\mathrm{BH}} / M_{\star}$; we recall that local samples suggest $\Gamma_{0} \approx 3 \times 10^{-3}$ with a small, but positive dependence on the stellar mass and with $1 \sigma$ spread $\approx 0.4$ dex.

Allowing for a Gaussian distribution of $\alpha_{\text {res }}$ around the average value 2 with a dispersion of 0.4 dex, we find that the bright tail $\left(L_{X} \gtrsim 10^{44} \mathrm{erg} \mathrm{s}^{-1}\right)$ of the luminosity function of X-ray selected AGNs is dominated by sources with $\alpha_{\text {res }} \gtrsim 5$ (see also Fig. 4 in Lapi et al. 2006). Such value is used in the computation of the lightcurves presented in Figs. 1 and 3. By further setting $\tau_{\mathrm{AGN}}=2 \times \tau_{\mathrm{ef}}$ and $\tau_{\mathrm{SFR}}=\tau_{\mathrm{ef}} / 3$ we obtain a good representation of the available statistics on AGNs detected in the FIR (see Fig. 10). Note that the SFR quenching timescale $\tau_{\mathrm{SFR}} \lesssim 10^{7} \mathrm{yr}$ is consistent with the estimate by Daddi et al. (2007) and Maiolino et al. (2012).

The AGN light curves as a function of the galactic time are then exploited to estimate the luminosity function at different redshift, properly taking into account the halo formation rate (see Appendix for details). The results shown in Fig. 11 indicate a very good agreement with the data at $z=2$ and 3.

We stress that moving toward lower luminosities the typical values of $\alpha_{\text {res }}$ approach the mean value $\approx 2$, so that these objects do not feature a prolonged declining phase. If they did, the low luminosity tails of the luminosity functions would exceed observational determinations (see also the discussion by Granato et al. 2006). Similar constraints also come from statistical matching arguments between the AGN accreted mass function and the local BH mass functions (see Yu \& Lu 2004). 
In the top panels of Fig. 12 the effect of changing the timescale for the decline of the SFR and of the AGN luminosity is illustrated. Keeping the same timescale for the star formation decline but reducing that of the nuclear activity would imply a much higher fraction of detected AGNs. The opposite is true when prolonging the duration of the nuclear activity. By the same token, if the star formation is switched off too early with respect to the AGN accretion, then the detection fraction significantly decreases, while it increases if significant star formation occurs during the last phases of AGN activity. The middle left panel of Fig. 12 shows that the uncertainty in the bolometric correction $k_{X}$ is a minor issue. However, it is instead relevant for reproducing the X-ray luminosity function (cf. Fig. 11; see also Lapi et al. 2006). The middle right panel elucidates the effect of changing the FIR luminosity threshold. We stress that the thresholds span a factor of 10, a wide range even compared with the expected uncertainty associated to the observational data. An uncertainty restricted to a factor of 2 is reasonably achievable with accurate analysis of the present data, and would allow us to derive firm conclusions on the way the star formation and the nuclear activity are switched off. Indeed, the figure highlights the potential of accurate follow-up at FIR/(sub-)mm restframe wavelengths of high- $z$, X-ray selected AGNs. The bottom left panel shows the effects of varying the parameter $\alpha_{\text {res }}$ and the final BH mass; increasing $\alpha_{\text {res }}$ causes more objects to feature the declining phase in the nuclear activity, and implies a smaller fraction of sources detected in the FIR-bright phase; the opposite holds when reducing $\alpha_{\text {res }}$. The bottom right panel illustrates the effects of varying the time when the decline of the star formation and nuclear activity sets in. Increasing this time makes the FIR-bright phase more prolonged, so raising the detection fraction, and viceversa.

The observed average FIR luminosity of the AGN hosts as a function of their X-ray luminosity is an additional test for the decline of the star formation and of the nuclear activity (see Fig. 13). We stress that the difference in redshift apparent in the model predictions is almost entirely due to different thresholds at different $z$, that reflect the data sampling. The statistics of these averages depend on the flux/luminosity distribution of detected and undetected sources around the X-ray detection threshold. It is clear that the X-ray primary selection picks out AGNs with $L_{\mathrm{FIR}} / L_{\mathrm{AGN}} \gtrsim 1$ for $L_{X} \lesssim 10^{44} \mathrm{erg} \mathrm{s}^{-1}$, while ratios $L_{\mathrm{FIR}} / L_{\mathrm{AGN}} \lesssim 1$ are typical at larger X-ray luminosity. Though caution is mandatory due to different detection limits, this behavior is nevertheless consistent with the fact that the declining phase of the X-ray luminosity is present on average in AGNs with $L_{X} \gtrsim 10^{44} \mathrm{erg} \mathrm{s}^{-1}$, i.e., in massive galaxies endowed with substantial BH masses. Meanwhile, in AGNs with $L_{X} \lesssim 10^{44} \mathrm{erg} \mathrm{s}^{-1}$ the turn off of the AGN activity occurs on a timescale of the same order or even smaller than the turn off in the star formation. All in all, the data suggest that the decline sets up at around $L_{\mathrm{FIR}} \sim L_{\mathrm{AGN}}$.

The faster decline of the star formation in luminous X-ray AGNs is also supported by the results of Page et al. (2012), who claimed the detection of a strong reduction in the SFR at increasing X-ray luminosity, by stacking AGN positions with flux derived from Herschel Multi-tiered Extragalactic Survey (HerMES) maps. This finding is not fully confirmed by Harrison et al. (2012), who remarks that within the error bars it is possible that the SFR keeps constant with increasing X-ray luminosity. However, their suggestion depends on an upper limit to the SFR at $L_{X} \approx 3 \times 10^{44}$ $\mathrm{erg} \mathrm{s}^{-1}$, larger by a factor of 3 with respect to that in Page et al. (2012).

It is worth noticing that, as pointed out by several authors (see Harrison et al. 2012; Mullaney et al. 2012b; Rosario et al. 2012), at high luminosities a contribution from the AGN could add to the power from the star formation. The amount depends on the wavelength of observation, since the AGN emission is less important at (sub-)mm than at FIR restframe wavelengths (Richards et al. 2006; Polletta et al. 2007; Cai et al. 2013).

Exploiting optical and near-IR photometry, Mullaney et al. (2012b) estimated the stellar mass in the host galaxy of AGNs with luminosity $10^{42} \lesssim L_{X} \lesssim 10^{44} \mathrm{erg} \mathrm{s}^{-1}$. They concluded that the FIR-detected host galaxies exhibit average stellar masses $M_{\star} \approx 5 \times 10^{10} M_{\odot}$, increasing by a factor of $\sim 2$ when the X-ray luminosity changes by about two order of magnitudes, and possibly tracing a power law $M_{\star} \propto L_{X}^{1 / 7}$ relationship. In the model the exponential growth of the AGN luminosity, coupled with the almost constant SFR, implies that the stellar mass depends only very weakly on $L_{\mathrm{AGN}}$ according to

$$
M_{\star}(t) \propto \dot{M}_{\star} \times \ln L_{\mathrm{AGN}}(t) .
$$

If mass estimates are available, the sSFR of the galaxies can be investigated. In Fig. 14 it is apparent that the model predicts a decrease in the specific star formation rate at large X-ray luminosities, $L_{X} \gtrsim 10^{44} \mathrm{erg} \mathrm{s}^{-1}$. This is a straightforward consequence of the decline in the SFR (see Fig. 13) during the final phase of the AGN and galaxy coevolution, when the stellar mass is already piled up. The present data do not show a clear trend. In particular we notice that the lack of data at high X-ray luminosity, $L_{X} \gtrsim 5 \times 10^{44} \mathrm{erg} \mathrm{s}^{-1}$, depends on the limited volume covered by current surveys (see Fig. 6 in Rovilos et al. 2012).

All studies of X-ray selected AGNs agree on very weak or no correlation between the X-ray absorbing column density and the FIR luminosity (e.g., Stevens et al. 2005; Shao et al. 2010; Lutz et al. 2010; Rosario et al. 2012; Rovilos et al. 2012). This result suggests that most of the X-ray absorption may originate not from the gas and dust directly involved in the star formation process, but be more related to the very central regions (see the review by Turner \& Miller 2009). The same conclusion has been indicated by direct studies of $z \sim 2$ heavily absorbed QSOs (Page et al. 2011).

On the other hand, Brusa et al. (2010) show that the fraction of obscured $\left(N_{\mathrm{H}} \gtrsim 10^{22} \mathrm{~cm}^{-2}\right)$ AGNs strongly decreases with increasing X-ray luminosity, and ranges from $\sim 80-90 \%$ at $L_{X} \sim 10^{42-43} \mathrm{erg} \mathrm{s}^{-1}$ to $\sim 10-20 \%$ at $L_{X} \gtrsim 6 \times 10^{45} \mathrm{erg} \mathrm{s}^{-1}$, with an abrupt transition around $L_{X} \sim 10^{44} \mathrm{erg} \mathrm{s}^{-1}$. For X-ray selected AGNs this luminosity corresponds, on average, to the transition from bolometric luminosity dominated by star formation to that dominated by nuclear activity (see Fig. 13). 
These findings can be understood recalling that an unbiased X-ray selection can pick up objects either before or after the SFR quenching. Specifically, in the early epoch dominated by star formation (see Fig. 1) the gas is abundant both in the reservoir and in the central regions of the galaxy, and both settings can contribute to the obscuration. When the AGN reaches its maximum power, the ISM is rapidly blown away by winds on a timescale $\sim \tau_{\text {ef }} / 3$ and the possible obscuration due to the ISM drastically decreases. On the other hand, the QSO winds may also affect the reservoir/proto-torus and the innermost X-ray absorbing regions, for instance peeling off the gas at higher latitude and reducing the covering factors.

Interestingly, the high fraction $\sim 40 \%$ of FIR detections in a sample of highly absorbed X-ray QSOs with $N_{\mathrm{H}} \gtrsim 10^{22}$ $\mathrm{cm}^{-2}$ and $L_{X} \gtrsim 10^{45} \mathrm{erg} \mathrm{s}^{-1}$ found at $z \sim 1.5-2$ by Stevens et al. (2005) can be understood on the same grounds. Since these authors select highly obscured QSOs, they on the average pick up objects that are still retaining a large reservoir and a large amount of gas in their ISM, and so are likely retaining a large SFR. Taking into account that the space density of absorbed QSOs is $\sim 15 \%$ of that of the unabsorbed ones at given X-ray luminosity (see Page et al. 2011 ), the $\sim 40 \%$ fraction of FIR detections for highly absorbed QSOs is still consistent with the low fraction $\lesssim 10 \%$ for an unbiased selection at large X-ray luminosity (Rosario et al. 2012; Page et al. 2012; Harrison et al. 2012).

The results of Stevens et al. (2005) are also supported by Mainieri et al. (2011) and by Carrera et al. (2011, 2013), who have shown that a major fraction of all obscured QSO hosts at $z \gtrsim 1$ are forming stars at significant rates. More quantitatively, the absorbed X-ray selected QSOs exhibit luminosity ratios in the range $0.3 \lesssim L_{\mathrm{FIR}} / L_{\mathrm{AGN}} \lesssim 5$ (Stevens et al 2005; Vignali et al. 2009; Gilli et al. 2011; Feruglio et al. 2011), supporting the view that they typically reach their large X-ray luminosity when star formation is still vigorous.

Inspecting Fig. 15, it is apparent that for obscured X-ray QSOs the observed ratios $L_{\mathrm{FIR}} / L_{\mathrm{AGN}}$ correspond to an interval in time $\sim 3 \tau_{\text {ef }}$ before the AGN decline phase sets in (see cyan box). Since during this epoch both the reservoir and the ISM are rich in gas and dust, X-ray obscured QSOs are also obscured in the UV-optical bands and, as a consequence, are on the average extremely 'red' (e.g., Fiore et al. 2009). After the onset of the QSO winds the SFR rapidly decreases and, as mentioned above, the covering factor of the reservoir also decreases. In fact, the population of X-ray unobscured QSOs exhibit $L_{\mathrm{FIR}} / L_{\mathrm{AGN}} \lesssim 0.3$ (e.g., Page et al. 2004) and they look similar to the pure optically selected QSOs (see below).

In conclusion, the above considerations illustrate that the study of star formation in well defined samples of X-ray selected AGNs can be extremely useful in statistically defining the observational framework to which a sensible theory of coevolution must conform. As a matter of fact, the X-ray selected objects may lie not only on the rising branch of the X-ray luminosity curve when the SFR is almost constant, but also in the declining phase of the SFR and nuclear activity. The number of objects with $L_{\mathrm{FIR}} / L_{\mathrm{AGN}} \gtrsim 1$ relative to that with $L_{\mathrm{FIR}} / L_{\mathrm{AGN}} \lesssim 1$ is an important clue on the timescale of the SFR quenching by the AGN feedback and on the timescale over which the nuclear activity itself is switching off.

\subsubsection{Star formation in optically selected QSOs}

Star formation in some of the galaxies hosting an optically selected QSO has been clearly detected in the (sub-)mm even at very high redshifts (see Omont et al. 1996, 2001, 2003; Carilli et al. 2001; Priddey et al. 2003; Wang et al. 2008; Serjeant et al. 2010; Bonfield et al. 2011; Mor et al. 2012), up to $z \sim 7$ (Venemans et al. 2012). Most of these observations have targeted QSOs endowed with very large bolometric luminosity $L_{\mathrm{AGN}} \approx 4 \times 10^{47} \mathrm{erg} \mathrm{s}^{-1}$.

Omont et al. (2001, 2003), Carilli et al. (2001), Priddey et al. (2003) observed more than one hundred optically selected QSOs $(2 \lesssim z \lesssim 4)$ at mm wavelengths, with a detection rate $\approx 30 \%$. At variance with the X-ray absorbed QSOs, the optically selected ones exhibit $L_{\mathrm{FIR}} / L_{\mathrm{AGN}} \lesssim 0.3$ with an average value $\sim 0.12$ for detected sources. Millimeter flux stacking on undetected targets yields $\left\langle L_{\mathrm{FIR}}\right\rangle /\left\langle L_{\mathrm{AGN}}\right\rangle \approx 1.5 \times 10^{-2}$. The typical average ratio for the detected and undetected QSOs is $\left\langle L_{\mathrm{FIR}}\right\rangle /\left\langle L_{\mathrm{AGN}}\right\rangle \approx 0.06$. Mor et al. (2012) detected in the sub-mm bands with Herschel five optically selected QSOs at $z \sim 4.8$, deriving larger FIR to X-ray luminosity ratios $0.25 \lesssim L_{\mathrm{FIR}} / L_{\mathrm{AGN}} \lesssim 0.6$. However, as pointed out by these authors, such values must be taken as upper limits, since the sub-mm fluxes refer to regions within 10 arcsec from the targeted QSOs and they are close to or below the source confusion limit. Small ratios of FIR to bolometric luminosity for high redshift QSOs are fully confirmed by the results of the Herschel-ATLAS survey (see Serjeant et al. 2010; Bonfield et al. 2011), which include also objects with lower bolometric luminosity, $L_{\mathrm{AGN}} \sim 10^{46} \mathrm{erg} \mathrm{s}^{-1}$.

The (sub-)mm searches have also been performed by targeting more than 60 optically selected QSOs at $z \sim 6$, once more favoring quite powerful sources, though there are already attempts to observe also QSOs fainter than $L_{\mathrm{AGN}} \lesssim 10^{47} \mathrm{erg} \mathrm{s}^{-1}$ (see Willott et al. 2007; Wang et al. 2008, 2010, 2011; Omont et al. 2013; Leipski et al. 2013). The fraction of detected luminous QSOs with $L_{\mathrm{AGN}} \approx 3 \times 10^{47} \mathrm{erg} \mathrm{s}^{-1}$ turns out to be $\approx 30 \%$, remarkably close to what is found at lower redshifts. The average maximum ratio $\left\langle L_{\mathrm{FIR}}\right\rangle /\left\langle L_{\mathrm{AGN}}\right\rangle \lesssim 0.3$, the minimum value $\approx 0.02$ derived by stacking undetected sources, and the average value $\approx 0.12$ for detected objects are quite close to those for lower redshift samples.

Can our model cope with the results for bright QSOs? The (positive) answer is in Fig. 16. We recall that in the model the bright QSOs are associated to massive galaxy halos formed at high redshift, in agreement with the clustering data (e.g., Hickox et al. 2011). If the optically bright QSOs on average appear at a time $t_{\text {opt }} \approx t_{\text {decl }}$ when $L_{\text {FIR }} / L_{\mathrm{AGN}} \lesssim 0.3$ (only a handful of observed QSOs exceed this limit), the subsequent evolution of the ratio predicted by the model is

$$
\frac{L_{\mathrm{FIR}}}{L_{\mathrm{AGN}}}\left(t \gtrsim t_{\mathrm{opt}}\right) \approx \frac{L_{\mathrm{FIR}}}{L_{\mathrm{AGN}}}\left(t_{\mathrm{opt}}\right) e^{-5 / 2 \times\left(t-t_{\mathrm{opt}}\right) / \tau_{\mathrm{ef}}} \approx 0.3 e^{-5 / 2 \times\left(t-t_{\mathrm{opt}}\right) / \tau_{\mathrm{ef}}} .
$$


The time lapse between the larger ratio $L_{\mathrm{FIR}} / L_{\mathrm{AGN}} \approx 0.3$ and the ratio $L_{\mathrm{FIR}} / L_{\mathrm{AGN}} \approx 0.02$ obtained by stacking non-detections amounts to $\approx 1.2 \tau_{\text {ef. }}$. This is an estimate of the time interval between the onset of the optical phase and the time when bright QSOs are on the average no longer detected at mm wavelengths. Over this time the optical luminosity decreases by a factor of $\approx 2$, while the FIR luminosity decreases by a factor of about 30 . According to Eq. (11), the average time interval during which the bright QSOs are detectable at mm wavelengths is given by $\sim 2 / 5 \times \ln (0.3 / 0.12) \tau_{\mathrm{ef}} \approx 0.36 \tau_{\mathrm{ef}}$, recalling that the corresponding $L_{\mathrm{FIR}} / L_{\mathrm{AGN}} \approx 0.12$. As a result, the expected detection fraction amounts to $0.36 / 1.2 \approx 30 \%$.

In this context, the similarity of the detected fraction $(\approx 30 \%)$ of bright QSOs at low and high redshift can be understood on the basis of the above equation, i.e., ascribed to the similarity of the physical processes in action at redshift $z \gtrsim 1.5$. Although the above estimates are somewhat uncertain since the statistics depend on the FIR detection threshold, nevertheless they highlight the potential of refined data on the ratio $L_{\mathrm{FIR}} / L_{\mathrm{AGN}}$ to trace this phase of the bright QSO evolution.

These results refer to QSOs with bolometric luminosity $L_{\mathrm{AGN}} \gtrsim 6 \times 10^{47} \mathrm{erg} \mathrm{s}^{-1}$. However, the detection rate decreases to $\lesssim 5-10 \%$ when fainter QSOs with magnitude $M_{1450} \gtrsim-25$, or $L_{\mathrm{AGN}} \lesssim 6 \times 10^{46}$ erg s s $^{-1}$ are considered, see Omont et al. (2013). The same authors find $\left\langle L_{\mathrm{FIR}}\right\rangle /\left\langle L_{\mathrm{AGN}}\right\rangle \approx 0.07$ for their observed 20 QSOs, in agreement with the findings for brighter ones. Willott et al. (2013) studied with ALMA two faint QSOs with $M_{1450} \gtrsim-25$; one of the objects has been detected and features $L_{\mathrm{FIR}} / L_{\mathrm{AGN}} \approx 0.05$, while the second one has not been detected, providing an upper limit $L_{\mathrm{FIR}} / L_{\mathrm{AGN}} \lesssim 0.02$. Also Bonfield et al. (2011) find that the fraction of detections decreases to $\approx 8 \%$ when lower luminosity QSOs $\left(L_{\mathrm{AGN}} \sim 10^{45} \mathrm{erg} \mathrm{s}^{-1}\right)$ are included in the target sample. In our framework two effects cooperate to get such results. First, at a given final $\mathrm{BH}$ mass, the decrease of the nuclear luminosity corresponds to a faster decrease of the SFR. Secondly, less luminous QSOs are on the average associated with less massive BHs and less massive halos and, as a consequence, with host galaxies exhibiting lower SFRs (see Fig. 16).

In Fig. 17 we show how the FIR detection fraction of optically selected QSOs depends on the model parameters. In particular, we illustrate how it changes when varying the timescales of the AGN (top left) and the SFR decline (top right), the bolometric correction (bottom left), and the FIR detection threshold (bottom right). The same comments to Fig. 12 hold, so we do not repeat them here.

The observational data and the model suggest that the X-ray absorbed QSOs and the optically selected QSOs are representative of two adjacent and subsequent phases, encompassing the time when the AGN luminosity overcomes the FIR luminosity of the host, while the QSO winds remove the ISM and reshape the reservoir around the BH (see Fig. 15). The handful of optically selected QSOs with $L_{\mathrm{FIR}} / L_{\mathrm{AGN}} \gtrsim 0.3$ can be understood as peculiar objects, wherein the obscuration usually associated with large FIR to AGN luminosity ratio does not preclude them to be selected in optical surveys. For instance, the QSO J0129-0035 at $z=5.78$ is the faintest one found in the SDSS and exhibits $L_{\mathrm{FIR}} / L_{\mathrm{AGN}} \approx 0.83$ (Wang et al. 2013). It also features a quite weak Ly $\alpha$ line emission (Jiang et al. 2009), as expected for a partially obscured object. On the other hand, we recall that when $L_{\mathrm{FIR}} / L_{\mathrm{AGN}} \lesssim 0.3$ the AGN feedback is fully removing the cold gas and stopping any flow toward the reservoir/torus, which shrinks down in mass and size. Therefore, during this epoch the optical and X-ray emissions are on the average less obscured (see $\S 5$ ). Thus the selected objects appear as unobscured AGNs, exhibiting the well known correlation between UV/Optical and X-ray emission (e.g., Lusso et al. 2010).

In our framework, luminous objects evolve along the following sequence: at first they are galaxies violently forming stars and growing up a supermassive BH in their centers. The BH manifests its mass accretion in hard X-rays because the obscuration for these energetic photons is less dramatic. Then the QSO winds clear up the ISM and an optical bright phase follows till the exhaustion of the fuel in the reservoir (see Fig. 3). The sequence looks similar to that suggested by Sanders et al. (1988), although in our model major mergers are not the leading phenomenon. It is quite remarkable that even at high redshift the stage of strong star formation in a QSO precursor has been already detected. This is the case of HFSL3 at $z \approx 6.3$ (Riechers et al. 2013 ). Its gas mass $M_{\text {gas }} \approx 10^{11} M_{\odot}$, about $40 \%$ of the dynamical mass, is distributed within a radius $\lesssim 2 \mathrm{kpc}$ and its properties are consistent with our scheme. Such system is predicted to evolve into a QSO like SDSSJ114816.64+525150.3 at $z \approx 6.42$ within a few $e$-folding times. As a matter of fact, in the latter object Walter et al. (2009) have detected a huge star formation rate $\dot{M}_{\star} \approx 1700 M_{\odot} \mathrm{yr}^{-1}$ within a radius $\lesssim 1 \mathrm{kpc}$. We notice that SDSSJ114816.64+525150.3 has a ratio $L_{\mathrm{FIR}} / L_{\mathrm{AGN}} \approx 0.2$, as expected in our framework (see Fig. 15). An additional interesting aspect is that the FIR selected galaxies exhibit larger abundance of low excitation gas with respect to the QSOs (e.g., Riechers et al. 2011), again possibly marking a step in the evolution.

From the above discussion we expect that the luminous optically selected QSOs are detectable for $\approx 1-2 e$-folding times around the maximum of their light curve. This visibility time is properly included in the computations of the optical luminosity functions at various redshifts. The results reported in Fig. 18 show a quite good agreement with the observations at high redshifts.

An additional prediction of our overall picture is that the mass in stars and in the BH are already settled to their final value in optically selected QSOs, since both star formation and $\mathrm{BH}$ accretion are exponentially declining during the optical bright phase. A discussion of this issue is deferred to $\S 5.1$. In conclusion, though the AGN light curve around and after the peak of the activity is modeled in order to fit the behavior of X-ray selected AGNs, it nicely describes also the statistics of optically selected QSOs.

\section{ADDITIONAL OBSERVATIONAL CONSTRAINTS}

There are additional observations, concerning the evolution of the BH to stellar mass ratio, the obscured to unobscured AGN fraction and the QSO outflows that are less systematic because of their inherent difficulty, but still 
provide useful constraints. In the following we discuss how the model confronts with these observations.

\subsection{Stellar and the BH masses in QSOs}

As recalled in $\S 1$, the mass in the old stellar population correlates with the mass of the central BH in local ETGs. On the other hand, it has been claimed by many authors that the relationship changes with cosmic time. While individual $\mathrm{BH}$ and stellar mass estimates for high redshift AGNs/QSOs must be taken with caution, the data are nevertheless rather informative.

The first relevant piece of evidence is that the optically selected QSOs at $z \gtrsim 2$ exhibit on the average an $M_{\mathrm{BH}} / M_{\star}$ mass ratio $3-20$ times higher than the local value, $\Gamma_{0} \approx 3 \times 10^{-3}$, (e.g., McLure \& Dunlop 2004; Peng et al. 2006; Coppin et al. 2008; Decarli et al. 2010; Merloni et al. 2010; Wang et al. 2010; Targett et al. 2012; Omont et al. 2013; Wang et al. 2013). The model envisages that such a finding is due to a selection bias. In fact, the high luminosity tail $\left(L_{\mathrm{AGN}} \gtrsim 10^{47} \mathrm{erg} \mathrm{s}^{-1}\right)$ of the luminosity function of optically selected AGNs/QSOs at high redshift $z \gtrsim 3$ comprises objects, that are on the high side $(\gtrsim 3 \sigma)$ of the local $\Gamma$ distribution (e.g., Wyithe \& Loeb 2003; Mahmood et al. 2005; Lapi et al. 2006, see their Fig. 4; Lauer et al. 2007). Considering the scatter of 0.4 dex of the local ratio, the values predicted by the model for optically selected QSOs is $\Gamma \gtrsim 10^{-2}$, in good agreement with the observational data.

Note that the model ascribes the large scatter in $\Gamma$ mainly to a scatter in $\alpha_{\text {res }}$, i.e., to variations in the amount of mass that is first accumulated in the reservoir and then accreted onto the BH. In addition, it is also possible that the average value of $\alpha_{\text {res }}$ may be itself a function of halo mass and redshift (see Eq. 8). The model predicts that optically selected QSOs have already assembled most of their final stellar and BH masses. This does not exclude that some mass is subsequently added, but the later evolution must have a minor impact on the average.

The complementary view of the $\mathrm{BH}$ and stellar mass evolution is given by (sub-)mm selected galaxies with detectable AGN activity. The results of Borys et al. (2005), Alexander et al. (2005, 2008), Melbourne et al. (2011), Carrera et al. (2011) strongly suggest that in these galaxies the ratio $\Gamma \lesssim(0.1-0.3) \Gamma_{0} \approx 3-9 \times 10^{-4}$ applies. This is consistent with the view that in (sub-)mm selected galaxies the BH mass is still piling up. The almost constant SFR coupled with the exponential increase of the $\mathrm{BH}$ accretion rate makes this ratio increasing almost exponentially before the declining phase. We stress that during this self-regulated regime of the $\mathrm{BH}$ growth, the ratio $\Gamma$ does not depend on the amount of matter stored in the reservoir, i.e., on $\alpha_{\text {res }}$. In relation to this point we recall that the stellar mass distribution estimated by Mullaney et al. (2012b) in X-ray selected AGNs as a function of the X-ray luminosity is well reproduced by the model.

\subsection{Fraction of obscured to unobscured AGNs/QSOs}

AGNs are mainly classified as obscured on the basis of emission line spectra, X-ray absorption $N_{\mathrm{H}} \gtrsim 10^{23} \mathrm{~cm}^{-2}$, and reddening $E(B-V)$. Such a classification criteria have been recently applied by several authors to objects selected in X-rays (see Bongiorno et al. 2012) or in the near-IR (Glikman et al. 2007; Banerji et al. 2012, 2013), with the purpose of understanding the fraction of obscured to unobscured AGNs/QSOs and of searching for clues on the connection between the obscuration and the host galaxy star formation activity. As usual, we are interested in high redshift sources, $z \gtrsim 1-1.5$. The fraction of red QSOs has been found to be significant. For instance Glikman et al. (2007) found that the fraction of reddened QSOs with $K \lesssim 14$ can range from $25 \%$ to $60 \%$ of the total underlying population. With different selection criteria on color and limiting magnitude Banerji et al. (2012) found that at very bright luminosities $M_{i} \lesssim-30$, the red QSOs are $\sim 5$ times more numerous than the UV bright ones. However, just below that limit the fraction decreases significantly to values close to those found by Glikman et al. (2007).

On the basis of the model we expect that an obscured AGN phenomenology occurs before the action of the QSO wind when $L_{\mathrm{FIR}} / L_{\mathrm{AGN}} \gtrsim 0.3$ and the UV-optical lines and continuum emission of the AGN are heavily absorbed by the reservoir/torus and by the dust-rich ISM. Contrarily, unobscured sources are more likely selected after the onset of the QSO wind, when the ISM and the reservoir have been significantly impoverished. If we assume that the phase singled out by the X-ray absorbed QSOs coincides with that of the red QSOs, then on the basis of Fig. 1 and the discussion in $\S 3.2 .2$, a rough estimation of a $2.5: 1$ proportion between red and UV-selected QSOs can be derived.

Before the setting of QSO winds, during the obscured phase, the size of the highly ionized region of the ISM surrounding the QSOs is limited, since most of the UV and ionizing photons are heavily absorbed. The small size of this region around ULASJ112001.48+064124.3 at $z \approx 7.1$ (the highest redshift QSO detected so far) claimed by Mortlock et al. (2011) can be ascribed to such an effect, as also supported by the radiative transfer simulations by Bolton et al. (2011). These authors found that the UV bright phase should have lasted for about $10^{7}$ yr. Since ULASJ112001.48+064124.3 exhibits $L_{\mathrm{FIR}} / L_{\mathrm{AGN}} \approx 3 \times 10^{-2}$, our model would predict a UV bright phase duration of about one $e$-folding time, which in the model at that redshift amounts to $\approx 2 \times 10^{7} \mathrm{yr}$. The agreement is reasonable, taking into account the uncertainties associated to the data and modelization. Interestingly, this example suggests that the early, dusty phase of QSOs at very high redshift can be easily probed by the James Webb Space Telescope with the NIRCam and MIRI instruments (Dickinson 2013), as it can be explored at lower redshift with the WISE All Sky Survey (Wright et al. 2010; Banerji et al. 2013).

\subsection{Large scale QSO outflows}

The AGN feedback on the ISM should manifest itself in terms of large outflows (see Fabian 2012 for a review), signalling the rapid expulsion of gas and the dramatic shutoff of the star formation. Large outflows have been observed 
in relatively nearby galaxies hosting an active nucleus (Feruglio et al. 2010; Rupke \& Veileux 2011; Sturm et al. 2011; Greene et al. 2012). At larger redshift $z \gtrsim 1$ QSOs winds associated with large mass outflows have been detected by several studies (e.g., Maiolino et al. 2001, 2004; D’Odorico et al. 2004). Optically thick gas moving with large velocity around QSOs up to a distance of $100 \mathrm{kpc}$ has been detected by Prochaska \& Hennawi (2009), possibly associated with quite massive outflows.

Broad-absorption line QSOs constitute the most basic and longstanding aspects of outflow phenomenology; linedriven outflows are expected in the form of winds that can form just above the accretion disk by a combination of radiation and gas/magnetic pressure (see Zubovas \& King 2013). In fact, massive outflows have been confirmed in such objects by many X-ray observations (see Brandt \& Gallagher 2000; Chartas et al. 2003, 2009). Recently, Borguet et al. (2013) detected the most energetic QSO outflow to date with a kinetic power of $\sim 10^{46} \mathrm{erg} \mathrm{s}^{-1}$ and an associated mass flow rate of $\sim 400 M_{\odot} \mathrm{yr}^{-1}$.

The most evident massive QSO outflow has been detected by Maiolino et al. (2012) with extremely accurate and detailed observations of the C[II] line in SDSSJ114816.64+525150.3 at $z=6.42$. These authors estimate an outflow rate $\dot{M}_{\text {out }} \approx 3000 M_{\odot} \mathrm{yr}^{-1}$ capable of removing the gas in the host galaxy within $6 \times 10^{6}$ yr. Outflow rates predicted by our model for massive BHs (see Fig. 19) range from several hundreds to several thousands $M_{\odot}$ yr ${ }^{-1}$; at given mass the rates depend also on redshift, chiefly through the specific binding energy entering Eqs. (2) and (3).

The model also requires that before the peak of the nuclear activity there are weaker galactic winds mainly driven by stellar feedback, as observed in some starforming galaxies at substantial redshift (Alexander et al. 2010; Genzel et al. 2012). Both AGN and stellar feedbacks can remove a lot of cold gas from the central regions of galaxies, though on different timescales. For instance in SDSSJ114816.64+525150.3 the dynamical mass within a $\sim 2.5 \mathrm{kpc}$ radius is about twice the gas mass (Wang et al. 2010). The results of Maiolino et al. (2012) suggest that the central regions of the host are deprived by about half of their mass in $\lesssim 10^{7}$ yr. So these outflows must affect the inner structure of the host galaxies, as pointed out by several authors (see Fan et al. 2008, 2010, 2013; Damjanov et al. 2009; Zubovas et al. 2013).

It is worth mentioning that, while usually the AGN feedback is invoked to turn off the star formation, the latter may occur in the outflowing shell (see Ishibashi \& Fabian 2012; Silk 2013; Zubovas et al. 2013). Since FIR selected samples show that the SFR is high even when the AGN luminosity (and hence the feedback) is low, this mechanism can originate only a fraction of the total stellar mass, while still relevant with respect to the size of the host galaxy (Ishibashi \& Fabian 2012). In particular, in the early phases of the evolution, the SN feedback is energetically larger than the AGN one, and the observations reveal large stellar mass in the presence of large gas abundance and weak AGN activity (i.e., low mass BH).

In summary, large outflows able to remove most of the ISM from AGN hosts are detected at least in one case and possibly in several others. We recall that even the short duration of the phase can significantly depress the statistics of outflow detection. For instance, in the case of SDSSJ114816.64+525150.3 the gas within a scale of $5-10 \mathrm{kpc}$ is going to be removed in $\approx 6 \times 10^{6} \mathrm{yr}$, which is $20 \%$ of the duration of the QSO optical phase.

\section{DISCUSSION}

Here we first discuss the observational evidence on, and properties of the circumnuclear structures in local AGNs, related to the presence of a massive gas reservoir as predicted by the model (see Fig. 3). Then by extrapolating the results of local observations to higher redshifts, we show that $A L M A$ high resolution imaging and coordinated X-ray high resolution observations of strongly lensed (sub-)mm selected galaxies can cast light on the epoch of stellar and $\mathrm{BH}$ mass growth. In the light of the above scenario, we point out several physical mechanisms operating on different scales that can lead to the accretion of a fraction of the ISM.

\subsection{The reservoir}

As shown in Fig. 3 the model predicts that around the central BH a gas reservoir soon sets up. Its formation stems from the requirement of funnelling gas of low angular momentum at a rate proportional to the SFR, while the BH accretes in a self-regulated regime. Such a structure could constitute a (proto-)torus around the BH of the same kind as that observed at low redshift and often called for in order to explain the diversity of unobscured and obscured AGNs (see $\S 4.2$ ). This torus/reservoir, with size ranging from a few to several tens of parsecs, has been studied in detail only in nearby AGNs. High spatial resolution observations reveal that molecular gas and dust is largely present (e.g., Muller-Sanchez et al. 2009; Krips et al. 2011; Diamond-Stanic \& Rieke 2012; Sani et al. 2012; Hönig et al. 2013), often accompanied by star formation (e.g., Davies et al. 2007; Storchi-Bergmann et al. 2012). The dust distribution looks quite complex, with the hot dust located in compact structures, while the warm one is more diffuse (see Hönig et al. 2013).

The studies of Kawakatu et al. (2007), Spaans \& Meijerink (2008) and Maiolino (2008) concluded that a reservoir/torus with mass $M_{\text {res }} \approx 10^{8}-10^{9} M_{\odot}$ extending to $\sim 100$ pc can be revealed up to redshift $z \lesssim 2$ by $A L M A$, thanks to its extraordinary sensitivity and exquisite spatial resolution. In fact, recently $A L M A$, though in a still incomplete technical configuration, has revealed $\mathrm{CO}, \mathrm{HCN}, \mathrm{HCO}^{+}$emission lines in the very central regions of a few nearby objects (see Combes et al. 2013; Fathi et al. 2013; Izumi et al. 2013).

In high redshift galaxies the mass in cold gas/dust relative to that in stars is expected to be much larger than in local AGNs. In fact, CO emission lines have been detected by ALMA in a number of high redshift $z \sim 2-3$ objects (see Weiss et al. 2013). In addition, the potential efficiency of ALMA for these studies is boosted up in the case of 
strongly lensed (sub-)mm galaxies. As predicted by Negrello et al. (2007, 2010; see also Blain et al. 2004) on the basis of the model, strongly lensed starforming galaxies can be efficiently selected with large area, relatively shallow (sub-)mm surveys.

In this context, Gonzalez Nuevo et al. (2012) have shown that about $10^{3}$ strongly lensed starforming galaxies at redshift $z \gtrsim 2$ can be easily extracted from the Herschel-ATLAS survey that covers $\sim 550 \mathrm{deg}^{2}$. About $5 \%$ of them are magnified by a factor $\gtrsim 10$ (see Lapi et al. 2012; Bussmann et al. 2013). Additional starforming, strongly lensed galaxies have been detected by the Herschel/HerMES (see Wardlow et al. 2013) and by the SPT survey of about $1200 \mathrm{deg}^{-2}$ at mm wavelengths (Vieira et al. 2013). Taking the Kawakatu et al. (2007, see their Fig. 2) model as a reference, one finds that lensing by factors $\gtrsim 10$ brings the apparent size of a reservoir with physical size $R_{\text {res }} \approx 100$ pc to $\approx 0.07$ arcsecs (or 0.015 arcsecs for a $R_{\text {res }} \approx 20$ pc physical size), well above the resolution achievable with $A L M A$ for almost all CO transition lines. Specifically, the CO emission lines with $J \geq 2$ are detectable in 12 hr at $5 \sigma$ for redshift $z \gtrsim 2$. $\mathrm{CO}(6-5)$ and $\mathrm{CO}(5-4)$ are detectable in about 4 hr (Kawakatu et al. 2007, see their Fig. 3). Even the HCN lines can be observed at least for higher $J$ (Kawakatu et al. 2007, see their Fig. 4). The C[II] 158 $\mu$ m line can also be excited in the molecular torus by a combination of the AGN emission and of the possible star formation (Pérez-Beaupuits et al. 2011). If so, ALMA in its final configuration will be able to produce detailed mapping of the $\mathrm{C}[\mathrm{II}]$ emission in the torus up to large redshifts.

We caution that the Kawakatu et al. (2007) model makes the classic assumption of a smooth, homogeneous dust distribution (e.g., Pier \& Krolik 1992; Granato \& Danese 1994; Efstathiou \& Rowan-Robinson 1995). Clumpy torus models may be more realistic, as demonstrated by mid-IR observations of silicate emission features in local AGNs (see Nenkova 2008a,b; Hönig et al. 2010; Kawaguchi \& Mori 2011; also Hönig 2013).

Efstathiou et al. (2013) presented a detailed analysis on the spectrum of IRAS 08572+3915, a nearby starburst galaxy endowed with an AGN. Combining new Herschel observations with previous near- and far-IR datasets they showed that the dusty torus illuminated by the AGN contributes about $90 \%$ of the total IR luminosity. They also demonstrated that the far-IR luminosity in the wavelength range $40 \lesssim \lambda \lesssim 800 \mu \mathrm{m}$ of a smooth torus is a factor $\lesssim 2.5$ lower than that of a clumpy torus. The measured total SED falls in between the smooth and the clumpy torus SEDs (cf. their Fig. 3). All in all, this result suggests that the the far-IR continuum emission of a smooth torus can be taken as a lower limit to the case of a clumpy torus.

As for the high-J CO emission lines, Kawakatu et al. (2007) find their results to agree with the outcomes from numerical simulations of 3-D, non-local thermal equilibrium radiative transfer in inhomogeneous dusty tori (see Wada \& Tomisaka 2005). In fact, recent observations of nearby Seyfert galaxies with Herschel (see Hailey-Dunsheath et al. 2012; Spinoglio et al. 2012; Pereira-Santaella et al. 2013) have revealed high-J CO emission lines roughly consistent with the expectations from homogeneous torus models. We also remark that mid-IR emission from clumpy tori, as modeled by Hönig \& Kishimoto (2010) and investigated by means of high-spatial resolution mid-IR spectro-photometry of Seyfert galaxies by Hönig et al. (2010), may be detectable with the James Webb Space Telescope even in highredshift galaxies provided that they are gravitationally lensed at magnifications $\gtrsim 10$ (see Kawakatu \& Ohsuga 2011).

Maps and velocity profiles of the molecular lines in strongly lensed starbursting galaxies will produce invaluable information on chemical composition, kinematic and mass of the reservoir during a crucial phase of galaxy evolution and $\mathrm{BH}$ accretion. In particular instances of quite large gravitational amplification we can even get estimates of the BH mass. These observations should be complemented by X-ray observations, which for magnification $\gtrsim 10$ will allow us to get a detailed view of the nuclear emission even for highly obscured and quite low luminosity objects (Matt et al. 2004; Georgantopoulos et al. 2011). The predicted intrinsic X-ray luminosity for typical lensed galaxies exceeds $L_{X} \gtrsim 10^{42} \mathrm{erg} \mathrm{s}^{-1}$; the magnification by factors $\gtrsim 10$ makes them easily detectable in pointed observations up to $z \gtrsim 3$ with Chandra and the next generation of X-ray instruments.

In conclusion, coordinated, high-resolution ALMA and X-ray observations offer the possibility to probe the reservoir/torus and the AGN activity in many tens of lensed, FIR-selected galaxies. The outcome will provide an enormous progress in understanding the formation and coevolution of stars and $\mathrm{BH}$ in primeval galaxies.

\subsection{The gas path from the ISM to the accretion disc}

5.2.1. From the ISM to the reservoir

The first step for the ISM gas in its path toward the central BH is the reservoir. The model assumes that, when the star formation is active in the host galaxy, some mechanism is able to drive a fraction of the ISM gas into the reservoir at a rate proportional to the SFR, according to Eq. (7). Several physical mechanisms can cause a fraction of the gas in galaxies to lose angular momentum and to pile up in the very central regions. A non exhaustive list includes gas drag, dynamical friction of gas plus star clumps, tidal fields, spiral waves, winds and bars, radiation drag (e.g., Norman \& Scoville 1988; Shlosman et al. 1989, 1990; Shlosman \& Noguchi 1993; Hernquist \& Mihos 1995; Noguchi 1999; Umemura 2001; Kawakatu \& Umemura 2002; Kawakatu et al. 2003; Thompson et al. 2005; Bournaud et al. 2007, 2011; Hopkins \& Quataert 2010, 2011). In general the presence of clumps, which may be generated by fragmentation of gas already organized in an unstable disc or by inflow of gas and star subclumps, tends to increase the efficiency of such mechanisms.

Since the loss of angular momentum is a requirement also for the cold gas to collapse into clouds and to fragment into stars, the starburst activity and the AGN fueling have been often associated. Gravitational torques acting on gaseous and stellar disks can induce radial flows of the ISM (e.g., Shlosman et al. 1989, 1990), thus increasing the central surface density, triggering star formation in the inner kpc scale and moving gas toward the 100 pc region. Such 
gravitational torques can be induced by external events, such as tidal encounters, orbital torques by satellites and minor/major mergers, or by internal instabilities related to infall, bars, asymmetries generated during the evolution of the gas plus star structure. Disc instabilities also grow gaseous clumps that migrate toward the central regions by dynamical friction (Shlosman \& Noguchi 1993). The clumps can then contribute to the formation of the bulge (Noguchi 1999; Genzel et al. 2010, 2011; Immeli et al. 2004; Ceverino et al. 2010; Bournaud et al. 2007, 2014). All these processes are expected to establish a kind of relationship between the rate of star formation, at least in the inner $\mathrm{kpc}$ scale, and the rate at which the gas is delivered toward the very central regions of the galaxy.

Hopkins \& Quataert $(2010,2011)$ have explored in detail with numerical simulations the effects of gravitational torques (externally or internally induced). They show that part of the ISM gas is shocked and dissipates energy and angular momentum, flowing toward the central regions. On scales of $1-30 \mathrm{pc}$ such a gas can reach large surface densities, $\sim 10^{11} M_{\odot} \mathrm{kpc}^{-2}$. This gas can be identified with the reservoir/torus introduced in our model.

In summary, there are several physical mechanisms that can plausibly reduce the angular momentum at least for a fraction of the diffuse ISM gas and drive it to migrate from kpc to $\lesssim 100 \mathrm{pc}$ scale at a rate proportional to the SFR of the host galaxy. This possibility is encased into Eqs. (7) and (8) of the model. We stress that data support a large variance in the coefficient $\alpha_{\text {res }}\left(M_{\mathrm{H}}, z\right)$ appearing there, which looks quite plausible given the suggested mechanisms and the results of numerical simulations.

\subsubsection{From the reservoir to the accretion disc}

Once low angular momentum gas has accumulated in the reservoir, additional loss/tranfer of angular momentum is required in order to bring the gas toward the accretion disc at sub-parsec scales. Some of the mechanisms at work on larger scales have been proposed to operate also on the smaller ones; for instance, bars-in-bars instabilities (Shlosman et al. 1989, 1990), gravitational interactions and dynamical friction in clumped disc (Kumar 1999) have been proposed.

Several authors have pointed out that in the circumnuclear regions on parsec scales the gas is very likely rich in metals and dust, prone to fragment in clumps and to form stars. On the one hand, the stellar feedback can remove part of the gas from the reservoir; on the other hand, it can favor the gas to flow toward the accretion disc (e.g., Wada \& Norman 2001, 2002; Thompson et al. 2005; Murray et al. 2005; Kawakatu \& Wada 2008). For instance, in the model proposed by Kawakatu \& Wada (2008) the gas turbulence supported by SN explosions transports angular momentum. As a result, a fraction of $\sim 10-30 \%$ of the gas flowing from larger scales can migrate toward the accretion disc. Also in the numerical simulations by Hopkins \& Quataert (2010) there is a correlation between SFR in the circumnuclear disc and the rate of accretion onto the BH. Thompson et al. (2005) find two possible classes of circumnuclear discs depending on the rate at which the gas is supplied: discs with star formation large enough to consume most of the available gas and with practically no accretion onto the central BH; alternatively, discs with large star formation only at their periphery, still leaving enough gas to ensure significant accretion.

Storchi-Bregmann et al. (2012) have detected star formation in NCG1068 on a scale of $\sim 100$ pc. Sani et al. (2012) confirmed this result for 4 additional nearby Seyfert galaxies, but concluded that in inner regions the physical conditions of the gas are not favorable to star formation. On the theoretical side, Begelman \& Shlosman (2009) pointed out that in the circumnuclear discs highly turbulent continuous flows are relatively stable against fragmentation.

A relevant point is that the star formation and its associated feedback in the reservoir should not be too much efficient; otherwise, the amount of gas available to flow toward the BH will be substantially reduced and eventually the relationship between stellar and $\mathrm{BH}$ mass will be erased. Likely, in the balance the overall mass of long living stars formed in the reservoir should be much less than the mass accreted onto the BH; this does not imply that the star formation is not important in helping the BH feeding, as mentioned above.

\subsubsection{The accretion rate and the effect of feedback}

In order to fit the observed statistics of both (sub-)mm/FIR and X-ray selected objects at $z \gtrsim 2$, the model assumes that the accretion is self-regulated with a time-averaged Eddington ratio $1 \lesssim \lambda \lesssim 4$, depending on the redshift, and constant mass to energy conversion efficiency $\epsilon \approx 0.15$. The model also envisages that, when the AGN feedback becomes dominant and the reservoir is no more fed, the accretion becomes supply-limited and sub-Eddington. These outcomes can be compared to the predictions from theoretical treatments and numerical simulations concerning the accretion onto the central BH.

Analytical treatments and hydrodynamical simulations have been used so far to explore possible effects of the AGN radiative power on the accretion rate (e.g., Begelman 1979; Abramowicz \& Lasota 1980; Abramowicz et al. 1988; Watarai et al. 2000; Watarai 2006; Ohsuga 2007; Ohsuga \& Mineshige 2011; Fabian 2012). In general, the rate $\dot{M}_{\text {accr }}$ of gas accretion onto the $\mathrm{BH}$ is possibly only a fraction of the rate $\dot{M}_{\text {inj }}$ at which the gas is supplied from the reservoir toward the BH, i.e., $\dot{M}_{\text {accr }}=f_{\text {accr }} \dot{M}_{\text {inj }}$ due to possible outflows (Begelman 2012; Watarai 2006; Ohsuga 2007; Li 2012); the net effect is a kind of self-regulation (see Debuhr et al. 2010, 2011, 2012). Depending on the local physical conditions, accretion and outflow rates, mass-to-radiation efficiencies and Eddington ratios are expected to fluctuate on very short time scales (e.g., Ohsuga 2007; Li 2012); we stress that in the model we refer to quantities averaged over the timescale needed by the $\mathrm{BH}$ to acquire its final mass.

In the model it has been assumed that most of the matter delivered from the reservoir toward the $\mathrm{BH}$ does accrete and only a negligible fraction goes into winds, i.e. $f_{\text {accr }} \approx 1$. Once more this is required in order not to lose the correlation between the $\mathrm{BH}$ and stellar mass. However, a significant variance in $f_{\text {accr }}$ is expected and should produce a variance in the final $\mathrm{BH}$ masses. 
Accretion rate and radiation efficiency are coupled in accretion discs and depend on the BH specific angular momentum (Abramowicz et al. 1988; Watarai 2006; Li 2012; Narayan et al. 2012; Roth et al. 2012; Li et al. 2013; Liu et al. 2013). The possibility that a regime of photon trapping can infringe the classical Eddington limit has been pointed out, together with the general conditions for its onset and decay (e.g., Blandford \& Begelman 2004; Begelman et al. 2006; Begelman \& Shoslman 2009; Begelman 2012).

Watarai (2006) presented useful approximations of numerical and analytical results for thick/thin disks in the case of super/sub-Eddington accretion. If the accretion rate is parameterized as $\xi=\dot{M}_{\text {accr }} c^{2} / L_{\mathrm{Edd}}$, then the Eddington ratio can be approximated by

$$
\lambda=\frac{L}{L_{\mathrm{Edd}}} \simeq 2 \ln \left[1+\frac{\epsilon_{0}}{2} \xi\right]
$$

where $\epsilon_{0} \approx 0.3$ is the efficiency for a maximally rotating $\mathrm{BH}$; we recall that the spin of the $\mathrm{BH}$ rapidly increases to this maximal value during super-Eddington accretion episodes. The radiative efficiency during accretion is bound to be $\epsilon=\lambda / \xi$. As a consequence, the accretion rate may exceed the Eddington one $(\xi>1)$, nevertheless keeping the ratio $\lambda \lesssim 4$ and the radiative efficiency $\epsilon \lesssim 0.15$.

How does our basic model compare with this theoretical framework? In Fig. 20 the value of accretion rate $\xi$, Eddington ratio $\lambda$ and radiative efficiency $\epsilon$ are presented as a function of galactic age for a QSO shining at $z=6$ with a bolometric luminosity $L_{\mathrm{AGN}} \approx 3 \times 10^{47} \mathrm{erg} \mathrm{s}^{-1}$ and associated to a halo mass $M_{\mathrm{H}} \approx 2 \times 10^{12} M_{\odot}$. The accretion rate implied by the model and the data is almost constant with $\xi \approx 25$ for $\approx 15 e$-folding times and then it decreases exponentially till the reservoir is exhausted. This opens the theoretical issue of finding mechanisms able to provide accretion rates exceeding the Eddington one, but up to such a value $\xi \approx 25$. Notice that in this case $\tau_{\text {ef }} \approx 1.8 \times 10^{7} \mathrm{yr}$ and the growth phase lasts $3 \times 10^{8} \mathrm{yr}$, less than the age of the Universe (amounting to $\sim 1 \mathrm{Gyr}$ at $z \sim 6$ ). Watarai's approximation yields $\lambda \approx 3$ and $\epsilon \approx 0.13$, very similar to the values adopted in the model (see Fig. 20). Similar results have been found by $\mathrm{Li}$ (2012), who has also explored the possibility of having a largely super-Eddington regime followed by a sub-Eddington one in order to explain the presence of the most massive $\mathrm{BH}, M_{\mathrm{BH}} \approx 10^{9} M_{\odot}$ at $z \approx 6$.

Figure 20 shows that the rapid decrease of the luminosity after the quasar wind and throughout the quasar optical phase, entails a rapid decrease of $\lambda$. This behavior can be compared with the Eddington ratios inferred for optically selected QSOs. The observed distribution of Eddington ratios $\lambda \equiv L_{\mathrm{AGN}} / L_{\mathrm{Edd}}$ in $z \approx 6$ QSOs estimated by Willott et al. (2010) exhibits a lognormal distribution peaked at $\lambda \approx 1$, with a dispersion $\approx 0.3$ dex; for comparison, the distribution of $z \approx 2$ QSOs peaks at $\lambda \approx 0.37$. The trend of decreasing Eddington ratio with redshift has been confirmed exploiting quite large samples by Kelly \& Shen (2013), who also found that for optical QSOs at $z \lesssim 5$ the Eddington ratio takes on a maximum value $\lambda \approx 3$. In the model during the exponential growth of the $\mathrm{BH}$ the maximum Eddington ratio is around $\lambda \approx 4$ at $z \approx 6$ and $\lambda \approx 1$ at $z \approx 2$, and decreases exponentially from those values during the optical bright phase (cf. Fig. 20), reproducing the observed behavior.

We notice that with $\xi \lesssim 25$, the radiative efficiency $\epsilon \approx 0.13$ is still large enough to have significant luminosity even during the super-Eddington phase. On the contrary, a too small efficiency would affect the fraction of nuclear activity detections in FIR selected objects (see Fig. 10). During the optical bright phase the efficiency $\epsilon=\lambda / \xi$ increases approaching the limit of 0.3. Unfortunately, observational determinations of the efficiency for individual AGNs are still problematic (Raimundo et al. 2012). We recall that $\lambda$ and $\epsilon$ are key parameters in the estimate of the mass accreted onto the supermassive BH during the bright AGN phase (e.g., Salucci et al. 1999; Marconi et al. 2004; Shankar et al. 2004, 2013: Kelly \& Merloni 2012), and average values $\epsilon \gtrsim 0.1$ are required by abundance matching arguments.

After the ejection of the cold gas by the QSO feedback, the mass in the reservoir, if not completely exhausted, is no longer sufficient to sustain a super-Eddington accretion (the BH mass is large); thus a supply-limited, subEddington accretion regime sets in during the declining phase of the AGN luminosity. We recall that the BH influence radius $\approx G M_{\mathrm{BH}} / \sigma_{\star}^{2}$ itself increases exponentially before the peak of the AGN activity, to attain values $\approx 70\left(M_{\mathrm{BH}} / 10^{9} M_{\odot}\right)\left(\sigma_{\star} / 250 \mathrm{~km} \mathrm{~s}^{-1}\right)^{-2} \mathrm{pc}$, close to the possible reservoir size. In these conditions, the standard theory of thin accretion discs should apply. A naive estimate of the accretion rate reads

$$
\dot{M}_{\mathrm{accr}}=\frac{M_{\mathrm{res}}}{\tau_{\mathrm{visc}}}=\frac{\sigma_{\star}^{3}}{G \mathcal{R}_{\mathrm{crit}}} \frac{M_{\mathrm{res}}}{M_{\mathrm{BH}}} ;
$$

here, according to standard prescriptions (e.g., Burkert \& Silk 2001; Begelman 2012; King 2012), the viscous timescale $\tau_{\text {visc }} \sim \mathcal{R}_{\text {crit }} \times \tau_{\text {dyn }}$ is taken as the dynamical time $\tau_{\text {dyn }} \approx G M_{\mathrm{BH}} / \sigma_{\star}^{2} \times 1 / \sigma_{\star}$ at the $\mathrm{BH}$ influence radius times the critical Reynolds number, $\mathcal{R}_{\text {crit }} \sim 10^{2-3}$, for the onset of turbulence.

However, the typical accretion rates derived from Eq. (13) are large; e.g., for the halo mass $M_{\mathrm{H}} \approx 6 \times 10^{12} M_{\odot}$ at $z \approx 2$ considered in Fig. 3 (corresponding to $\sigma_{\star} \approx 250 \mathrm{~km} \mathrm{~s}^{-1}$ ), we have at the beginning of the declining phase a mass ratio $M_{\text {res }} / M_{\mathrm{BH}} \approx 3$, hence the viscous accretion rate amounts to $\dot{M}_{\text {accr }} \sim 10^{2}\left(\mathcal{R}_{\text {crit }} / 10^{2}\right)^{-1} M_{\odot} \mathrm{yr}^{-1}$. The accretion rates required by the data on the FIR detected fractions in X-ray selected AGN samples are lower than the simple limits derived above, amounting to $\dot{M}_{\text {accr }} \sim 5(\epsilon / 0.15)^{-1} M_{\odot} \mathrm{yr}^{-1}$. This may indicate that the fueling mechanism is very complex, in that it does not depend solely on the amount of gas still available after the QSO ejection, but also on various other physical conditions. In fact, gas and dust spatial distribution, magnetic fields, viscosity, cooling and heating, radiative pressure and additional aspects have been recently introduced in hydrodynamical simulations to capture the main features of the mass transfer toward the central BH (see Narayan et al. 2012; Roth et al. 2012; Li 
et al. 2013; Liu et al. 2013). The results we have derived from observations in the present paper can help toward further, educated investigations.

\section{SUMMARY AND CONCLUSIONS}

We have exploited the recent, wide samples of FIR selected galaxies followed-up in X rays and of X-ray/optically selected AGNs followed-up in the FIR band, along with the classic AGN and stellar luminosity functions at $z \gtrsim 1.5$, to infer the following scenario for the coevolution of supermassive BHs and massive host galaxies $\left(M_{\mathrm{H}} \gtrsim 10^{12} \widetilde{M}_{\odot}\right)$ :

- the SFR in the host galaxy remains approximately constant for a time $\Delta t_{\text {burst }} \sim 0.5-1$ Gyr and then declines abruptly because of the QSO feedback on a very short time scale $\tau_{\mathrm{SFR}} \approx \tau_{\mathrm{ef}} / 3 \approx \Delta t_{\mathrm{burst}} / 40$;

- part of the central ISM loses angular momentum and reaches the circumnuclear regions at a rate $\dot{M}_{\text {inflow }}=$ $\alpha_{\text {res }} \times 10^{-3} \dot{M}_{\star}$, with typical values $\alpha_{\text {res }} \approx 2$ and scatter $\sim 0.4$ dex reflecting those of the local BH to stellar mass ratio;

- the early accretion onto a small seed BH, $M_{\text {seed }} \sim 10^{2-3} M_{\odot}$, occurs in a self-regulated regime with efficiency $\epsilon \approx 0.15$ and radiative power that can be just above the Eddington limit $\lambda \lesssim 4$, especially at the highest redshifts; thus the BH grows exponentially with an $e$-folding time $\tau \sim$ a few to several $10^{7} \mathrm{yr}$;

- during the growth of the $\mathrm{BH}$ a massive reservoir forms, with a size of $20-100 \mathrm{pc}$, close to the influence radius of the $\mathrm{BH}$ at the end of its evolution; the reservoir mass is as large as the final $\mathrm{BH}$ mass, ready to be delivered to the $\mathrm{BH}$ in the last $2-3 e$-folding times;

- in the case of massive BHs the QSO feedback at its maximum exceeds the stellar feedback and is able to remove the ISM, stopping the star formation and the fueling of the reservoir; if the reservoir has enough gas a phase of supply-limited accretion follows, with luminosity (and Eddington ratio) exponentially declining over a timescale $\tau_{\mathrm{AGN}} \approx 2 \tau_{\mathrm{ef}}$.

Interestingly, the time evolution of both the host galaxy and the AGN can be characterized in terms of number of $e$-folding times; the galaxy/AGN coevolution takes about 15 -folding times of the BH growth (see Figs. 1 and 3 ).

We have computed the FIR luminosity of the host galaxy and the bolometric luminosity of the AGN/QSO as a function of the galactic age for different formation redshifts and halo masses (see Figs. 1 and 3). We have exploited them in association with the statistics of halo formation to reproduce:

- the luminosity function of FIR selected, high redshift galaxies (see Fig. 2), as well as the stellar mass function of their descendants, i.e., the passively evolving ETGs (see Fig. 4);

- the luminosity function of X-ray and optically selected AGN/QSOs (see Figs. 11 and 18);

- the available statistics on X-ray detections of FIR selected galaxies, and on FIR detections of X-ray/optically selected AGNs (see Figs. 5, 6, 7, 8, 10, 13, 14, 16);

- the statistics of BH to stellar mass ratio in FIR selected galaxies and optically selected QSOs (see $\S 4.1$ ).

We stress that, in order to comply with the current FIR and X-ray data, any model of coevolution must feature the time evolution of the SFR and $\mathrm{BH}$ accretion rate outlined above, plus a relationship between SFR and halo mass as a function of redshift in the line of Eq. (4). We also stress the importance of obtaining additional, accurate and well defined statistics on the SFR in X-ray selected AGNs/QSOs and on the nuclear activity in FIR selected galaxies, via coordinated observations from current and next generation (sub-)mm and X-ray instruments. These observations will be crucial to follow in greater detail the different stages of the AGN/galaxy coevolution process, and in particular to pinpoint the ratio between the duration timescale of the star formation and the $e$-folding timescale of the $\mathrm{BH}$ accretion.

We have found that the ratio $L_{\mathrm{FIR}} / L_{\mathrm{AGN}}$ is the observational parameter that characterizes the time evolution of the galaxy plus AGN system (see Fig. 15, top panel); it is a decreasing function of the galactic time and marks the evolution from the epoch when the luminosity budget is dominated by the star formation to the epoch when the AGN/QSO takes over. The FIR selection elicits objects with $L_{\mathrm{FIR}} / L_{\mathrm{AGN}} \gtrsim 0.3$ and $\mathrm{BH}$ to stellar mass ratios $\Gamma$ smaller than the average local value $\Gamma_{0} \approx 3 \times 10^{-3}$. The primary X-ray selection picks out objects spanning the whole range of the luminosity ratio; in particular, the X-ray QSOs with large obscuration exhibit $0.3 \lesssim L_{\text {FIR }} / L_{\mathrm{AGN}} \lesssim 5$. Instead, the optically selected QSOs show $0.01 \lesssim L_{\mathrm{FIR}} / L_{\mathrm{AGN}} \lesssim 0.3$, marking the decrease of the star formation on a short timescales and the epoch of the reservoir exhaustion on longer timescales; the bright objects feature a $\mathrm{BH}$ to stellar mass ratio $\Gamma \gtrsim \Gamma_{0}$.

Our findings indicate the following scenario (see Fig. 15, bottom panel): AGNs in massive halos begin their life as faint and obscured nuclei; then they evolve into obscured QSOs visible mostly in the X-ray or in mid-IR bands, and eventually become bright QSOs visible even in the optical band. In this framework the role of the dust is crucial; it must form soon, at the beginning of the host evolution, in order to yield the appropriate FIR luminosity, and then it has to be largely removed at the beginning of the optical bright AGN phase, in a couple of $e$-folding times. The 
nuclear activity is detectable in hard X-ray (rest-frame) energies for longer times, amounting to $4-5 e$-folding times; this is in line with the increasing mean free path of the X-ray photons with increasing energy.

The time evolution of the star formation and $\mathrm{BH}$ accretion envisaged by our model can be compared with other semi-analytical and numerical works appeared in the recent literature. Khandai et al. (2012; see also Springel et al. 2005, Sijacki et al. 2009) exploited numerical simulations including galaxy mergers and cold gas streams to investigate the growth of galaxies and hosted supermassive BHs at high redshift. Their light-curves (see Fig. 2 of Khandai et al. 2012) feature an early phase along which both the SFR and the BH accretion rate rise, with the latter extremely rapid in attaining a few $\times 10^{-1} M_{\odot} \mathrm{yr}^{-1}$; as a result, the AGN bolometric luminosity exceeds $\sim 10^{45} \mathrm{erg} \mathrm{s}^{-1}$ over a rather long period when the SFR keeps sustained at more than $100 M_{\odot} \mathrm{yr}^{-1}$, so as to produce a very high fraction of X-ray detected AGNs in FIR selected hosts. Subsequently, after the action of the AGN feedback, their light curves feature a mild, slow decline in the SFR, with the BH accretion still at appreciable values, so as to imply a high fraction of FIR detections in X-ray/optically selected AGNs. Such high fractions are clearly at variance with current observational data (see $\S 3$ ). Part of these problems can be ascribed to: assumption of Bondi accretion strictly ceiled by the Eddington limit, the ensuing requirements on large $\mathrm{BH}$ seed masses $\gtrsim 10^{5} M_{\odot}$, and the recipe for mild AGN feedback on the large-scale star formation activity.

Fanidakis et al. (2012; see also Cole et al. 2000, Baugh et al. 2005) exploit a semi-analytic model that includes $\mathrm{BH}$ accretion by galaxy merging and disk instabilities to compute the evolution of AGNs across cosmic times. Their prescriptions (see Eqs. 1 and 2 of Fanidakis et al. 2012) imply a direct proportionality between the BH accretion rate and the SFR. With their adopted values for the fraction of stellar mass produced in the starburst that is accreted onto the $\mathrm{BH}$ (their parameter $f_{\mathrm{BH}} \approx 5 \times 10^{-3}$ ) and for the ratio between the accretion and the bulge dynamical timescale (their parameter $f_{q} \sim 1-10$ ), the resulting ratio of FIR to AGN bolometric luminosity stays almost constant with time at values $L_{\mathrm{FIR}} / L_{\mathrm{AGN}} \approx 2 / f_{q} \sim 0.2-2$. This again implies a very high X-ray detected fraction in FIR selected hosts. The problem can be mostly ascribed to the absence of the reservoir in their model, and to a prominent role of mergers in triggering the star formation and $\mathrm{BH}$ activity.

We have discussed the fueling of the reservoir ( $\S 5.2 .1$ and 5.2.2) and the physics of BH accretion (see $\S 5.2 .3$ ) in the light of numerical and analytic models. We have shown that observations can be accounted for by models predicting a self-regulated, super-Eddington accretion $\xi=\dot{M}_{\text {accr }} c^{2} / L_{\mathrm{Edd}} \approx 25$, associated to a radiative power only slightly super-Eddington $\lambda=L / L_{\text {Edd }} \lesssim 4$ and to a radiative efficiency $\epsilon \lesssim 0.15$. However, this phase cannot be too short to comply with the data, i.e., $\xi$ must not exceed $\sim 25$ by far. This opens the theoretical issue of finding mechanisms able to provide accretion rates exceeding the Eddington rate, but up to a maximum value. Subsequently, the exponential decline of the luminosity required by the data implies the Eddington ratio $\lambda$ to decrease. Correspondingly, following the prescription of Eq. (12) by Watarai (2000) and $\operatorname{Li}(2012)$ the radiative efficiency $\epsilon=\lambda / \xi$ is expected to rapidly increase up to $\epsilon \sim 0.3$ (see dotted line in Fig. 20). Numerical simulations honed for this task can cast light on physical mechanisms at work to originate such a behavior.

One of our major predictions is the formation of a reservoir in the circumnuclear regions; the reservoir hosted by typical star forming galaxies with $\dot{M}_{\star} \gtrsim 200 M_{\odot} \mathrm{yr}^{-1}$ reaches a mass $M_{\text {res }} \gtrsim 3 \times 10^{8} M_{\odot}$. In $\S 5.1$ we have pointed out that these hosts are easily selected by (sub-)millimeter surveys such as Herschel-ATLAS, HerMES and those performed with the SPT. Many tens of these hosts are strongly lensed with amplification factors $\gtrsim 10$ by intervening galaxies. In such cases, coordinated, high-resolution observations in the (sub-)mm band with $A L \widetilde{M} A$ and in the X-ray band with Chandra and the next generation of X-ray telescopes will allow us to study in detail the evolution of the supermassive $\mathrm{BH}$ and of its reservoir.

This work has been supported in part by the MIUR PRIN 2010/2011 'The dark Universe and the cosmic evolution of baryons: from current surveys to Euclid', by the INAF PRIN 2012/2013 'Looking into the dust-obscured phase of galaxy formation through cosmic zoom lenses in theHerschel Astrophysical Terahertz Large Area Survey', by ASI/INAF agreement No. I/072/09/0, and by the INAF PRIN 2009 'New light on the early universe with submillimeter spectroscopy'. We are grateful to the anonymous referee for helpful comments and suggestions, and to A. Cavaliere, G.L. Granato, M. Massardi, P. Salucci for interesting discussions. A.L. thanks SISSA for warm hospitality.

\section{APPENDIX}

\section{APPENDIX. COMPUTING DETECTED FRACTIONS AND LUMINOSITY FUNCTIONS}

In this Appendix we detail how we compute some quantities of interest in the present work.

The fraction of objects $f_{\mathrm{FIR} \rightarrow \mathrm{X}}$ selected in the FIR with luminosity $L_{\mathrm{FIR}}$ within the bin $\left[L_{\mathrm{FIR}}^{\mathrm{INF}}, L_{\mathrm{FIR}}^{\mathrm{SUP}}\right]$ that are detected in $\mathrm{X}$ rays with an AGN luminosity $L_{\mathrm{X}}$ above the limit $L_{\mathrm{X}}^{\mathrm{LIM}}$ is computed as:

$$
f_{\mathrm{FIR} \rightarrow \mathrm{X}} \equiv \frac{\int \mathrm{d} M_{\mathrm{H}} \mathrm{d} N / \mathrm{d} M_{\mathrm{H}} \Delta t_{\mathrm{FIR} \rightarrow \mathrm{X}}}{\int \mathrm{d} M_{\mathrm{H}} \mathrm{d} N / \mathrm{d} M_{\mathrm{H}} \Delta t_{\mathrm{FIR}}} .
$$

Here $\mathrm{d} N / \mathrm{d} M_{\mathrm{H}}\left(M_{\mathrm{H}}, z_{\text {form }}\right)$ is the halo mass function, $\Delta t_{\mathrm{FIR}}\left[M_{\mathrm{H}}, z_{\text {form }}\right]$ is the time the object spends at FIR luminosity $L_{\mathrm{FIR}}^{\mathrm{INF}}<L_{\mathrm{FIR}}\left[t \mid M_{\mathrm{H}}, z_{\text {form }}\right]<L_{\mathrm{FIR}}^{\mathrm{SUP}}$ while $\Delta t_{\mathrm{FIR} \rightarrow \mathrm{X}}\left(M_{\mathrm{H}}, z_{\text {form }}\right)$ is the time the object spends at FIR luminosity $L_{\mathrm{FIR}}^{\mathrm{INF}}<$ $L_{\mathrm{FIR}}\left[t \mid M_{\mathrm{H}}, z_{\text {form }}\right]<L_{\mathrm{FIR}}^{\mathrm{SUP}}$ with X-ray luminosity $L_{X}\left[t \mid M_{\mathrm{H}}, z_{\text {form }}\right]>L_{X}^{\mathrm{LIM}}$ above the detection limit. The average of a 
given quantity $Q\left[t \mid M_{\mathrm{H}}, z_{\text {form }}\right]$ among the detected sources is computed as

$$
<Q>_{\mathrm{FIR} \rightarrow \mathrm{X}} \equiv \frac{\int \mathrm{d} M_{\mathrm{H}} \mathrm{d} N / \mathrm{d} M_{\mathrm{H}} \int_{\Delta t_{\mathrm{FIR} \rightarrow \mathrm{X}}} \mathrm{d} t Q(t)}{\int \mathrm{d} M_{\mathrm{H}} \mathrm{d} N / \mathrm{d} M_{\mathrm{H}} \Delta t_{\mathrm{FIR}}} .
$$

The same formalism applies when computing the fraction of objects $f_{\mathrm{X} \rightarrow \mathrm{FIR}}$ selected in $\mathrm{X}$ rays and detected in the FIR, or the fraction of objects $f_{\mathrm{O} \rightarrow \mathrm{FIR}}$ selected in the optical band (dominated by the QSO emission) and detected in the FIR.

In addition, the luminosity function in a given observational band is computed as

$$
\Phi(\log L, t)=\int^{t} \mathrm{~d} t_{\text {form }} \int \mathrm{d} \log M_{\mathrm{H}} \frac{\mathrm{d}^{2} N}{\mathrm{~d} t_{\text {form }} \mathrm{d} \log M_{\mathrm{H}}} \frac{e^{-\log ^{2}\left(L / L\left[t \mid M_{\mathrm{H}}, t_{\text {form }}\right]\right) / 2 \sigma_{\log L}^{2}}}{\sqrt{2 \pi \sigma_{\log L}^{2}}} .
$$

In the above expression, $\mathrm{d}^{2} N / \mathrm{d} t_{\text {form }} \mathrm{d} \log M_{\mathrm{H}}$ is the halo formation rate as given by Lapi et al. (2013), $L\left[t \mid M_{\mathrm{H}}, t_{\text {form }}\right]$ is the lightcurve from the model at observation time $t$ for a halo of mass $M_{\mathrm{H}}$ and formation time $t_{\text {form }}$, and a lognormal distribution of the luminosity around the average value $L\left[t \mid M_{\mathrm{H}}, t_{\mathrm{form}}\right]$ with scatter $\sigma_{\log L}$ has been assumed. For further details we refer the reader to Lapi et al. $(2006,2011)$ and Cai et al. (2013).

\section{REFERENCES}

Abramowicz M.A., \& Lasota J.P. 1980, Acta Astron., 30, 35

Abramowicz, M.A., Czerny, B., Lasota, J.P., \& Szuszkiewicz, E. 1988, ApJ, 332, 646

Aird, J., Nandra, K., Laird, E.S., et al. 2010, MNRAS, 401, 2531

Aird, J., Nandra, K., Georgakakis, A., Laird, E.S., Steidel, C.C., \& Sharon, C. 2008, MNRAS, 387, 883

Alexander, D.M., \& Hickox, R.C. 2012, New Astron. Rev., 56, 93

Alexander, D.M., Swinbank, A.M., Smail, I., McDermid, R., \& Nesvadba, N.P.H. 2010, MNRAS, 402, 2211

Alexander, D.M., Brandt, W.N., Smail, I., et al. 2008, AJ, 135, 1968

Alexander, D.M., Bauer, F.E., Chapman, S.C., Smail, I., Blain, A. W., Brandt, W.N., \& Ivison, R.J. 2005, ApJ, 632, 736

Antonucci, R. 1993, ARA\&A, 31, 473

Ballo, L., Cristiani, S., Fasano, G., et al. 2007, ApJ, 667, 97

Banerji, M., McMahon, R.G., Hewett, P.C., Gonzalez-Solares, E., \& Koposov, S.E. 2013, MNRAS, 429, L55

Banerji, M., McMahon, R.G., Hewett, P.C., Alaghband-Zadeh, S., Gonzalez-Solares, E., Venemans, B.P., \& Hawthorn, M.J. 2012 MNRAS, 427, 2275

Basu-Zych, A.R., Lehmer, B.D., Hornschemeier, A.E., et al. 2013 , ApJ, 762, 45

Baugh C.M., Lacey C.G., Frenk C.S., Granato G.L., Silva L., Bressan A., Benson A.J., \& Cole S., 2005, MNRAS, 356, 1191

Begelman M.C. 2012, ApJ, 749, L3

Begelman M.C. 1979, MNRAS, 187, 237

Begelman, M.C., \& Shlosman, I. 2009, ApJ, 702, L5

Begelman, M.C., Volonteri, M., \& Rees, M.J. 2006, MNRAS, 370, 289

Beifiori, A., Courteau, S., Corsini, E.M., \& Zhu, Y. 2012, MNRAS, 419, 2497

Bennert, V.N., Auger, M.W., Treu, T., Woo, J.-H., \& Malkan, M.A. 2011, ApJ, 742, 107

Blain, A.W., Chapman, S.C., Smail, I., \& Ivison, R.J. 2004, ApJ, 611,725

Blandford, R.D., \& Begelman, M.C. 2004, MNRAS, 349, 68

Bolton, J.S., Haehnelt, M.G., Warren, S.J., Hewett, P.C., Mortlock, D.J., Venemans, B.P., McMahon, R.G., \& Simpson, C. 2011, MNRAS, 416, L70

Bonfield, D.G., Jarvis, M.J., Hardcastle, M.J., et al. 2011, MNRAS, 416, 13

Bongiorno, A., Merloni, A., Brusa, M., et al. 2012, MNRAS, 427, 3103

Borys, C., Smail, I., Chapman, S.C., Blain, A.W., Alexander, D. M., \& Ivison, R.J. 2005, ApJ, 635, 853

Borguet, B.C.J., Arav, N., Edmonds, D., Chamberlain, C., \& Benn, C. 2013, ApJ, 762, 49

Bournaud, F., Perret, V., Renaud, F., et al. 2014, ApJ, 780, 57

Bournaud, F., Dekel, A., Teyssier, R., et al. 2011, ApJ, 741, L33

Bournaud, F., Elmegreen, B.G., \& Elmegreen, D.M. 2007, ApJ, 670,237

Bouwens, R. J., Illingworth, G. D., Oesch, P. A., et al. 2011, ApJ, 737,90

Brandt, W. N., \& Gallagher, S. C. 2000, New Astron. Rev., 44, 461
Brusa, M., Civano, F., Comastri, A., et al. 2010, ApJ, 716, 348

Burkert, A., \& Silk, J. 2001, ApJ, 554, L151

Bussmann, R.S., Pérez-Fournon, I., Amber, S., et al. 2013, ApJ, 779,25

Cai, Z.-Y., Lapi, A., Xia, J.-Q., et al. 2013, ApJ, 768, 21

Carilli, C.L., Bertoldi, F., Rupen, M.P., et al. 2001, ApJ, 555, 625

Carrera, F.J., Ali, A.K., Page, M.J., Symeonidis, M., Stevens, J.A., \& Cao Orjales, J.M. 2013, in Nuclei of Seyfert galaxies and QSOs - Central engine and conditions of star formation, in press [preprint arXiv:1302.2844]

Carrera, F.J., Page, M.J., Stevens, J.A., Ivison, R.J., Dwelly, T., Ebrero, J., \& Falocco, S. 2011, MNRAS, 413, 2791

Cavaliere, A., \& Vittorini, V. 2000, ApJ, 543, 599

Ceverino, D., Dekel, A., \& Bournaud, F. 2010, MNRAS, 404, 2151

Chabrier, G. 2003, PASP, 115, 763

Chartas, G., Saez, C., Brandt, W.N., Giustini, M., Garmire, G.P. 2009, ApJ, 706, 644

Chartas, G., Brandt, W. N., \& Gallagher, S. C. 2003, ApJ, 595, 85 Cirasuolo, M., McLure, R.J., Dunlop, J.S., Almaini, O., Foucaud, S., \& Simpson, C. 2010, MNRAS, 401, 1166

Clements, D.L., Rigby, E., Maddox, S., et al. 2010, A\&A, 518, L8

Cole, S., Lacey, C.G., Baugh, C.M., \& Frenk, C.S. 2000, MNRAS, 319, 168

Combes, F., García-Burillo, S., Casasola, V., et al. 2013, A\&A, 558, A124

Cook, M., Lapi, A., \& Granato, G.L. 2009, MNRAS, 397, 534

Coppin, K.E.K., Swinbank, A.M., Neri, R., et al. 2008, MNRAS, 389,45

Cristiani, S., 2004, Ap\&SS, 294, 79

Croom, S.M., Richards, G.T., Shanks, T., et al. 2009, MNRAS, 399, 1755

Daddi, E., Alexander, D.M., Dickinson, M., et al. 2007, ApJ, 670, 173

Damjanov, I., McCarthy, P.J., Abraham, R.G., et al. 2009, ApJ, 695,101

Davies, R.I., Müller Sánchez, F., Genzel, R., Tacconi, L.J., Hicks, E.K.S., Friedrich, S., \& Sternberg, A. 2007, ApJ, 671, 1388

Debuhr, J., Quataert, E., \& Ma, C.-P. 2012, MNRAS, 420, 2221

Debuhr, J., Quataert, E., \& Ma, C.-P. 2011, MNRAS, 412, 1341 Debuhr, Jackson; Quataert, Eliot; Ma, Chung-Pei

Debuhr, J., Quataert, E., Ma, C.-P., \& Hopkins, P. 2010, MNRAS, 406, L55

Decarli, R., Falomo, R., Treves, A., Labita, M., Kotilainen, J.K., \& Scarpa, R. 2010, MNRAS, 402, 2453

Diamond-Stanic, A.M., \& Rieke, G.H. 2012, ApJ, 746, 168

Dickinson, M. 2013, AAS Meeting, 221, 135.01

D'Odorico, V., Cristiani, S., Romano, D., Granato, G.L., \& Danese, L. 2004, MNRAS, 351, 976

Driver, S.P., Robotham, A.S.G., Bland-Hawthorn, J., et al. 2013, MNRAS, 430, 2622

Eales, S.A., Raymond, G., Roseboom, I.G., et al. 2010, A\&A, 518, L23

Efstathiou, A., Pearson, C., Farrah, D., et al. 2013, MNRAS, in press [preprint arXiv:1309.7005] 
Efstathiou, A., \& Rowan-Robinson, M. 1995, MNRAS, 273, 649

Elmegreen, B.G., Bournaud, F., \& Elmegreen, D.M. 2008, ApJ, 688,67

Fabian, A.C. 2012, ARA\&A, 50, 455

Fabian, A.C. 1999, MNRAS, 308, L39

Fan, L., Chen, Y., Er, X., Li, J., Lin, L., \& Kong, X. 2013, MNRAS, 431, L15

Fan, L., Lapi, A., Bressan, A., Bernardi, M., De Zotti, G., \& Danese, L. 2010, ApJ, 718, 1460

Fan, L., Lapi, A., De Zotti, G., \& Danese, L. 2008, ApJ, 689, L101

Fan, X., Strauss, M.A., Richards, G.T., et al. 2006, AJ, 131, 1203

Fanidakis, N., Baugh, C.M., Benson, A.J., et al. 2012, MNRAS, 419, 2797

Fardal, M.A., Katz, N., Weinberg, D.H., \& Davé, R. 2007, MNRAS, 379,985

Fathi, K., Lundgren, A.A., Kohno, K., et al. 2013, ApJ, 770, L27

Ferrarese, L., \& Ford, H. 2005, Sp.Sc.Rev., 116, 523

Ferrarese, L., \& Merritt, D. 2000, ApJ, 539, L9

Feruglio, C., Daddi, E., Fiore, F., Alexander, D.M., Piconcelli, E., \& Malacaria, C. 2011, ApJ, 729, L4

Feruglio, C., Maiolino, R., Piconcelli, E., Menci, N., Aussel, H., Lamastra, A., \& Fiore, F. 2010, A\&A, 518, L155

Fiore, F., Puccetti, S., Grazian, A., et al. 2012a, A\&A, 537, 16

Fiore, F., Puccetti, S., \& Mathur, S. 2012b, Adv. in Astron., vol. 2012,9

Fiore, F., Puccetti, S., Brusa, M., et al. 2009, ApJ, 693, 447

Fontanot, F., Cristiani, S., Monaco, P., Nonino, M., Vanzella, E., Brandt, W.N., Grazian, A., \& Mao, J. 2007, A\&A, 461, 39

Frenk, C.S., \& White, S.D.M. 2012, Annalen der Physik, 524, 507

Fukugita, M., \& Peebles, P.J.E. 2004, ApJ, 616, 643

Gebhardt, K., Kormendy, J., Ho, L.C., et al. 2000a, ApJ, 543, L5

Gebhardt, K., Bender, R., Bower, G., et al. 2000b, ApJ, 539, L13

Genzel, R., Tacconi, L.J., Combes, F., et al. 2012, ApJ, 746, 69

Genzel, R., Newman, S., Jones, T., et al. 2011, ApJ, 733, 101

Genzel, R., Eisenhauer, F.,\& Gillessen, S. 2010, Rev.Mod.Phys., 82,3121

Georgantopoulos, I., Rovilos, E., \& Comastri, A. 2011, A\&A, 526, 46

Gilli, R., Su, J., Norman, C., et al. 2011, ApJ, 730, L28

Glikman, E., Helfand, D.J., White, R.L., Becker, R.H., Gregg, M.D., \& Lacy, M. 2007, ApJ, 667, 673

González-Nuevo, J., Lapi, A., Fleuren, S., et al. 2012, ApJ, 749, 65

Graham, A.W., Onken, C.A., Athanassoula, E., \& Combes, F. 2011, MNRAS, 412, 2211

Graham, A.W. 2007, MNRAS, 379, 711

Graham, A.W., \& Driver, S.P. 2007, ApJ, 655, 77

Graham, A.W., Erwin, P., Caon, N., \& Trujillo, I. 2001, ApJ, 563, L11

Granato, G.L., Silva, L., Lapi, A., Shankar, F., De Zotti, G., \& Danese, L. 2006, MNRAS, 368, L72

Granato, G.L., De Zotti, G., Silva, L., Bressan, A., \& Danese, L. 2004, ApJ, 600, 580

Granato, G.L., Silva, L., Monaco, P., Panuzzo, P., Salucci, P., De Zotti, G., \& Danese, L. 2001, MNRAS, 324, 757

Granato, G.L., \& Danese, L. 1994, MNRAS, 268, 235

Greene, J.E., Zakamska, N.L., \& Smith, P.S. 2012, ApJ, 746, 86

Gruppioni, C., Pozzi, F., Rodighiero, G., et al. 2013, MNRAS, 432, 23

Gruppioni, C., Pozzi, F., Andreani, P., et al. 2010, A\&A, 518, L27

Gültekin, K., Richstone, D.O., Gebhardt, K., et al. 2009, ApJ, 698, 198

Guo, Q., White, S., Li, C., \& Boylan-Kolchin, M. 2010, MNRAS, 404, 1111

Hailey-Dunsheath, S., Sturm, E., Fischer, J., et al. 2012, ApJ, 755, 57

Häring, N., \& Rix, H.-W. 2004, ApJ, 604, L89

Harrison, C.M., Alexander, D.M., Mullaney, J.R., et al. 2012, ApJ, 760, L15

Hernquist, L., \& Mihos, J.C. 1995, ApJ, 448, 41

Hickox, R.C., Myers, A.D., Brodwin, M., et al. 2011, ApJ, 731, 117

Hönig, S.F. 2013, in Torus Workshop 2012, eds. C. Packham, R. Mason, and A. Alonso-Herrero, in press [preprint arXiv:1301.1349

Hönig, S.F., Kishimoto, M., Tristram, K.R.W., et al. 2013, ApJ, 771,87

Hönig, S.F., \& Kishimoto, M. 2010, A\&A, 523, A27

Hönig, S. F., Kishimoto, M., Gandhi, P., et al. 2010, A\&A, 515, A23
Hopkins, P.F., \& Quataert, E. 2011, MNRAS, 415, 1027

Hopkins, P.F., \& Quataert, E. 2010, MNRAS, 407, 1529

Hopkins, P.F., Richards, G.T., \& Hernquist, L. 2007, ApJ, 654, 731

Hunt, M.P., Steidel, C.C., Adelberger, K.L., \& Shapley, A.E. 2004, ApJ, 605, 625

Ilbert, O., McCracken, H.J., Le Fèvre, O., et al. 2013, A\&A, 556, 55

Immeli, A., Samland, M., Gerhard, O., \& Westera, P. 2004, A\&A, 413,547

Ishibashi, W., \& Fabian, A.C. 2012, MNRAS, 427, 2998

Izumi, T., Kohno, K., Martn, S., et al. 2013, PASJ, 65, 100

Jiang, L., Fan, X., Bian, F., et al. 2009, AJ, 138, 305

Johnson, S.P., Wilson, G.W., Wang, Q.D., et al. 2013, MNRAS, 431,662

Johnson, J.L., Whalen, D.J., Li, H., \& Holz, D.E. 2013, ApJ, 771, 116

Khandai, N., Feng, Y., DeGraf, C., Di Matteo, T., \& Croft, R.A.C. 2012, MNRAS, 423, 2397

Kaviraj, S., Cohen, S., Windhorst, R.A., et al. 2013, MNRAS, 429, L40

Kawaguchi, T., \& Mori, M. 2011, ApJ, 737, 105

Kawakatu, N., \& Ohsuga, K. 2011, MNRAS, 417, 2562

Kawakatu, N., \& Wada, K. 2008, ApJ, 681, 73

Kawakatu, N., Andreani, P., Granato, G.L., \& Danese, L. 2007, ApJ, 663, 924

Kawakatu, N., Umemura, M., \& Mori, M. 2003, ApJ, 583, 85

Kawakatu, N., \& Umemura, M. 2002, MNRAS, 329, 572

Kelly, B.C., \& Shen, Y. 2013, ApJ, 764, 45

Kelly, B.C., \& Merloni, A. 2012, Adv. in Astron., vol. 2012, 7

King, A.R., 2012, Mem. S.A.It., 83, 466

King, A.R. 2010, MNRAS, 402, 1516

King, A.R. 2005, ApJ, 635, L121

King, A.R. 2003, ApJ, 596, L27

Kormendy, J., \& Ho, L.C. 2013, ARA\&A, 51, 511

Kormendy, J., \& Bender, R. 2013, ApJ, 769, L5

Kormendy, J., \& Bender, R. 2009, ApJ, 691, L142

Kormendy, J., \& Richstone, D. 1995, ARA\&A, 33, 581

Krips, M., Martín, S., Eckart, A., et al. 2011, ApJ, 736, 37

Kulier, A., Ostriker, J.P., Natarajan, P., Lackner, C.N., \& Cen, R. 2013, ApJ, submitted [preprint arXiv:1307.3684]

Kumar, P. 1999, ApJ, 519, 599

La Franca, F., Fiore, F., Comastri, A., et al. 2005, ApJ, 635, 864

Laird, E.S., Nandra, K., Pope, A., \& Scott, D. 2010, MNRAS, 401, 2763

Lapi, A., Salucci, P., \& Danese, L. 2013, ApJ, 772, 85

Lapi, A., Negrello, M., González-Nuevo, J., Cai, Z.-Y., De Zotti, G., \& Danese, L. 2012, ApJ, 755, 46

Lapi, A., González-Nuevo, J., Fan, L., et al. 2011, ApJ, 742, 24

Lapi, A., \& Cavaliere, A. 2011, ApJ, 743, 127

Lapi, A., Shankar, F., Mao, J., Granato, G.L., Silva, L., De Zotti, G., \& Danese, L. 2006, ApJ, 650, 42

Lauer, T.R., Gebhardt, K., Faber, S.M., et al. 2007, ApJ, 664, 226

Leipski, C., Meisenheimer, K., Walter, F., et al. 2013, ApJ, 772, 103

Li, J., Ostriker, J., \& Sunyaev, R. 2013, ApJ, 767, 105

Li, L.-X. 2012, MNRAS, 424, 1461

Li, C., \& White, S.D.M. 2009, MNRAS, 398, 2177

Liu, C., Yuan, F., Ostriker, J.P., Gan, Z., Yang, X. 2013, MNRAS, 434,1721

Lusso, E., Comastri, A., Vignali, C., et al. 2010, A\&A, 512, 34

Lutz, D., Mainieri, V., Rafferty, D., et al. 2010, ApJ, 712, 1287

Magorrian, J., Tremaine, S., Richstone, D., et al. 1998, AJ, 115, 2285

Mahmood, A., Devriendt, J.E.G., \& Silk, J. 2005, MNRAS, 359, 1363

Mainieri, V., Bongiorno, A., Merloni, A., et al. 2011, A\&A, 535, 80

Maiolino, R., Gallerani, S., Neri, R., et al. 2012, MNRAS, 425, L66

Maiolino, R. 2008, New Astron. Rev., 52, 339

Maiolino, R., Oliva, E., Ghinassi, F., Pedani, M., Mannucci, F., Mujica, R., \& Juarez, Y. 2004, A\&A, 420, 889

Maiolino, R., Marconi, A., Salvati, M., Risaliti, G., Severgnini, P., Oliva, E., La Franca, F., \& Vanzi, L. 2001, A\&A, 365, 28

Mao, J., Lapi, A., Granato, G.L., de Zotti, G., \& Danese, L. 2007, ApJ, 667, 655

Marchesini, D., Whitaker, K.E., Brammer, G., et al. 2010, ApJ, 725,1277

Marchesini, D., van Dokkum, P.G., Förster Schreiber, N.M., Franx, M., Labbé, I., \& Wuyts, S. 2009, ApJ, 701, 1765 
Marconi, A., \& Hunt, L.K. 2003, ApJ, 589, L21

Marconi, A., Risaliti, G., Gilli, R., Hunt, L.K., Maiolino, R., \& Salvati, M. 2004, MNRAS, 351, 169

Masters, D., Capak, P., Salvato, M., et al. 2012, ApJ, 755, 169

Matt, G., Bianchi, S., Dovčiak, M., Guainazzi, M., \& Karas, V. 2004, Prog. Theor. Phys, 155, 381

McConnell, N.J., \& Ma, C.-P. 2013, ApJ, 764, 184

McConnell, N.J., Ma, C.-P., Gebhardt, K., et al. 2011, Nature, 480, 215

McLure, R.J., Dunlop, J.S., Bowler, R.A.A., et al. 2013, MNRAS, 432, 2696

McLure, R.J., \& Dunlop, J.S. 2004, MNRAS, 352, 1390

Melbourne, J., Peng, C.Y., Chien Y., et al. 2011, AJ, 141, 141

Merloni, A., Bongiorno, A., Bolzonella, M., et al. 2010, ApJ, 708, 137

Mor, R., Netzer, H., Trakhtenbrot, B., Shemmer, O., \& Lira, P. 2012, ApJ, 749, L25

Mortlock, D.J., Warren, S.J., Venemans, B.P., et al. 2011, Nature, 474,616

Moster, B.P., Naab, T., \& White, S.D.M. 2013, MNRAS, 428, 3121

Moster, B.P., Somerville, R.S., Maulbetsch, C., van den Bosch, F.C., Macciò, A.V., Naab, T., \& Oser, L. 2010, ApJ, 710, 903

Moustakas, J., Coil, A.L., Aird, J., et al. 2013, ApJ, 767, 50

Mullaney, J.R., Daddi, E., Béthermin, M., et al. 2012a, ApJ, 753, L30

Mullaney, J.R., Pannella, M., Daddi, E., et al. 2012b, MNRAS, 419, 95

Müller Sánchez, F., Davies, R.I., Genzel, R., Tacconi, L.J., Eisenhauer, F., Hicks, E.K.S., Friedrich, S., \& Sternberg, A. 2009, ApJ, 691, 749

Murray, N., Quataert, E., \& Thompson, T.A. 2005, ApJ, 618, 569

Narayan, R., SÄdowski, A., Penna, R.F., \& Kulkarni, A.K. 2012, MNRAS, 426, 3241

Negrello, M., Hopwood, R., De Zotti, G., et al. 2010, Science, 330, 800

Negrello, M., Perrotta, F., González-Nuevo, J., et al. 2007 , MNRAS, 377, 1557

Nenkova, M., Sirocky, M.M., Nikutta, R., Ivezic, Z., \& Elitzur, M. 2008a, ApJ, 685, 160

Nenkova, M., Sirocky, M.M., Ivezic, Z., \& Elitzur, M. 2008b, ApJ, 685,147

Noguchi, M. 1999, ApJ, 514, 77

Norman, C., \& Scoville, N. 1988, ApJ, 332, 124

Oesch, P.A., Bouwens, R.J., Illingworth, G.D., et al. 2013, ApJ, 773,75

Ohsuga, K., \& Mineshige, S. 2011, ApJ, 736, 2

Ohsuga, K. 2007, ApJ, 659, 205

Omont, A., Willott, C.J., Beelen, A., Bergeron, J., Orellana, G., \& Delorme, P. 2013, A\&A, 552, 43

Omont, A., Beelen, A., Bertoldi, F., Cox, P., Carilli, C.L., Priddey, R.S., McMahon, R.G., \& Isaak, K.G. 2003, A\&A, 398, 857

Omont, A., Cox, P., Bertoldi, F., McMahon, R.G., Carilli, C., \& Isaak, K.G. 2001, A\&A, 374, 371

Omont, A., McMahon, R.G., Cox, P., Kreysa, E., Bergeron, J., Pajot, F., \& Storrie-Lombardi, L.J. 1996, A\&A, 315, 1

Page, M.J., Symeonidis, M., Vieira, J., et al. 2012, Nature, 485 , 213

Page, M.J., Carrera, F.J., Stevens, J.A., Ebrero, J., \& Blustin, A.J. 2011, MNRAS, 416, 2792

Page, M.J., Stevens, J.A., Ivison, R.J., \& Carrera, F.J. 2004, ApJ, 611, L85

Pei, Y.C. 1995, ApJ, 438, 623

Peng, C.Y., Impey, C.D., Ho, L.C., Barton, E.J., \& Rix, H.-W. 2006, ApJ, 640, 114

Pereira-Santaella, M., Spinoglio, L., Busquet, G., et al. 2013, in Torus Workshop 2012, eds. C. Packham, R. Mason, and A. Alonso-Herrero, in press [preprint arXiv:1301.3365]

Pérez-Beaupuits, J.P., Wada, K., \& Spaans, M. 2011, ApJ, 730, 48

Perrotta, F., Magliocchetti, M., Baccigalupi, C., et al. 2003, MNRAS, 338, 623

Pier, E.A., \& Krolik, J.H. 1992, ApJ, 401, 99

Planck Collaboration 2013, A\&A, submitted [preprint arXiv:1303.5076

Polletta, M., Tajer, M., Maraschi, L., et al. 2007, ApJ , 663, 81

Pota, V., Graham, A.W., Forbes, D.A., Romanowsky, A.J., Brodie, J.P., \& Strader, J. 2013, MNRAS, 433, 235

Priddey, R.S., Isaak, K.G., McMahon, R.G., \& Omont, A. 2003 MNRAS, 339, 1183
Prochaska, J.X., \& Hennawi, J.F. 2009, ApJ, 690, 1558

Rafferty, D.A., Brandt, W.N., Alexander, D.M., Xue, Y.Q., Bauer, F.E., Lehmer, B.D., Luo, B., \& Papovich, C. 2011, ApJ, 742, 3

Raimundo, S.I., Fabian, A.C., Vasudevan, R.V., Gandhi, P., \& Wu, J. 2012, MNRAS, 419, 2529

Richards, G.T., Strauss, M.A., Fan, X., et al. 2006, AJ, 131, 2766

Richards, G.T., Croom, S.M., Anderson, S.F., et al. 2005, MNRAS, 360,839

Riechers, D.A., Bradford, C.M., Clements, D.L., et al. 2013 , Nature, 496, 329

Riechers, D.A., Hodge, J., Walter, F., Carilli, C.L., \& Bertoldi, F. 2011, ApJ, 739, L31

Rosario, D.J., Santini, P., Lutz, D., et al. 2012, A\&A, 545, 45

Ross, N.P., McGreer, I.D., White, M., et al. 2013, ApJ, 773, 14

Roth, N., Kasen, D., Hopkins, P.F., \& Quataert, E. 2012, ApJ, 759, 36

Rovilos, E., Comastri, A., Gilli, R., et al. 2012, A\&A, 546, 58

Rupke, D.S.N., \& Veilleux, S. 2011, ApJ, 729, L27

Sadoun, R., \& Colin, J. 2012, MNRAS, 426, L51

Salpeter, E.E. 1964, ApJ, 140, 796

Salucci, P., Szuszkiewicz, E., Monaco, P., \& Danese, L. 1999, MNRAS, 307, 637

Sanders, D.B., Soifer, B.T., Elias, J.H., Madore, B.F., Matthews, K., Neugebauer, G., \& Scoville, N.Z. 1988, ApJ, 325, 74

Sani, E., Davies, R.I., Sternberg, A., et al. 2012, MNRAS, 424, 1963

Sani, E., Marconi, A., Hunt, L.K., \& Risaliti, G. 2011, MNRAS, 413, 1479

Santini, P., Rosario, D.J., Shao, L., et al. 2012, A\&A, 540, 109

Scannapieco, C., Wadepuhl, M., Parry, O.H., et al. 2012, MNRAS, 423, 1726

Serjeant, S., Bertoldi, F., Blain, A.W., et al. 2010, A\&A, 518, L7

Shankar, F., Weinberg, D.H., \& Miralda-Escudé, J. 2013, MNRAS, 428,421

Shankar, F. 2009, New Astron. Rev. 53, 57

Shankar, F., Lapi, A., Salucci, P., De Zotti, G., \& Danese, L. 2006, ApJ, 643, 14

Shankar, F., Salucci, P., Granato, G.L., De Zotti, G., \& Danese, L. 2004, MNRAS, 354, 1020

Shao, L., Lutz, D., Nordon, R., et al. 2010, A\&A, 518, L26

Shen, Y. 2013, Bull. Astron. Soc. India, 41, 61

Shlosman, I., \& Noguchi, M. 1993, ApJ, 414, 474

Shlosman, I., Begelman, M.C., \& Frank, J. 1990, Nature, 345, 679

Shlosman, I., Frank, J., \& Begelman, M.C. 1989, Nature, 338, 45

Sijacki, D., Springel, V., \& Haehnelt, M.G. 2009, MNRAS, 400, 100

Silk, J. 2013, ApJ, 772, 112

Silk, J., \& Rees, M.J. 1998, A\&A, 331, L1

Silva, L., Granato, G.L., Bressan, A., \& Danese, L. 1998, ApJ, 509, 103

Simmons, B.D., Van Duyne, J., Urry, C.M., et al. 2011, ApJ, 734 121

Soltan, A. 1982, MNRAS, 200, 115

Spaans, M., \& Meijerink, R. 2008, ApJ, 678, L5

Spinoglio, L., Pereira-Santaella, M., Busquet, G., et al. 2012, ApJ 758,108

Springel, V., Di Matteo, T., \& Hernquist, L. 2005, MNRAS, 361, 776

Stark, D.P., Ellis, R.S., Bunker, A., et al. 2009, ApJ, 697, 1493

Stevens, J.A., Page, M.J., Ivison, R.J., Carrera, F.J., Mittaz, J.P.D., Smail, I., \& McHardy, I.M. 2005, MNRAS, 360, 610

Storchi-Bergmann, T., Riffel, R.A., Riffel, R., Diniz, M.R., Borges Vale, T., \& McGregor, P.J. 2012, ApJ, 755, 87

Sturm, E., González-Alfonso, E., Veilleux, S., et al. 2011, ApJ, 733, L16

Symeonidis, M., Georgakakis, A., Seymour, N., et al. 2011, MNRAS, 417, 2239

Symeonidis, M., Rosario, D., Georgakakis, A., Harker, J., Laird, E.S., Page, M.J., \& Willmer, C.N.A. 2010, MNRAS, 403, 1474

Targett, T.A., Dunlop, J.S., \& McLure, R.J. 2012, MNRAS, 420, 3621

Thompson, T.A., Quataert, E., \& Murray, N. 2005, ApJ, 630, 167

Treister, E., Schawinski, K., Volonteri, M., Natarajan, P., \& Gawiser, E. 2011, Nature, 474, 356

Tremaine, S., Gebhardt, K., Bender, R., et al. 2002, ApJ, 574, 740

Turner, T.J., \& Miller, L. 2009, A\&A Rev., 17, 47

Ueda, Y., Akiyama, M., Ohta, K., \& Miyaji, T. 2003, ApJ, 598, 886 
Umemura, M. 2001, ApJ, 560, L29

Urry, C.M., \& Padovani, P. 1995, PASP, 107, 803

Vale, A., \& Ostriker, J.P. 2004, MNRAS, 353, 189

Vasudevan, R.V., \& Fabian, A.C. 2007, MNRAS, 381, 1235

Venemans, B.P., McMahon, R.G., Walter, F., et al. 2012, ApJ, 751, L25

Vestergaard, M., \& Osmer, P.S. 2009, ApJ, 699, 800

Vestergaard, M. 2004, ApJ, 601, 676

Vieira, J.D., Marrone, D.P., Chapman, S.C., et al. 2013, Nature, 495,344

Vieira, J.D., Crawford, T.M., Switzer, E.R., et al. 2010, ApJ, 719, 763

Vignali, C., Pozzi, F., Fritz, J., et al. 2009, MNRAS, 395, 2189

Volonteri, M. 2010, A\&A Rev, 18, 279

Wada, K., \& Tomisaka, K. 2005, ApJ, 619, 93

Wada, K., \& Norman, C.A. 2002, ApJ, 566, L21

Wada, K., \& Norman, C.A. 2001, ApJ, 547, 172

Walter, F., Riechers, D., Cox, P., Neri, R., Carilli, C., Bertoldi, F., Weiss, A., \& Maiolino, R. 2009, Nature, 457, 699

Wang, J., Navarro, J.F., Frenk, C.S., et al. 2011, MNRAS, 413, 1373

Wang, R., Wagg, J., Carilli, C.L., et al. 2013, ApJ, 773, 44

Wang, R., Wagg, J., Carilli, C.L., et al. 2011, AJ, 142, 101

Wang, R., Carilli, C.L., Neri, R., et al. 2010, ApJ, 714, 699

Wang, R., Carilli, C.L., Wagg, J., et al. 2008, ApJ, 687, 848

Wang, S.X., Brandt, W.N., Luo, B., et al. 2013, ApJ, 778, 179
Wardlow, J.L., Cooray, A., De Bernardis, F., et al. 2013, ApJ, 762, 59

Watarai, K.-Y. 2006, ApJ, 648, 523

Watarai, K.-Y., Fukue, J., Takeuchi, M., \& Mineshige, S. 2000, PASJ, 52, 133

Weiss, A., De Breuck, C., Marrone, D.P., et al. 2013, ApJ, 767, 88

White, S.D.M., \& Frenk, C.S. 1991, ApJ, 379, 52

Willott, C.J., Omont, A., \& Bergeron, J. 2013, ApJ, 770, 13

Willott, C.J. 2011, ApJ, 742, L8

Willott, C.J., Delorme, P., Reylé, C., et al. 2010, AJ, 139, 906

Willott, C.J., Delorme, P., Omont, A., et al. 2007, AJ, 134, 2435

Wolf, C., Wisotzki, L., Borch, A., Dye, S., Kleinheinrich, M., \& Meisenheimer, K. 2003, A\&A, 408, 499

Wright, E.L., Eisenhardt, P.R.M., Mainzer, A.K., et al. 2010, AJ, 140,1868

Wyithe, J.S.B., \& Loeb, A. 2003, ApJ, 595, 614

Xia, J.-Q., Negrello, M., Lapi, A., De Zotti, G., Danese, L., \& Viel, M. 2012, MNRAS, 422, 1324

Xue, Y.Q., Brandt, W.N., Luo, B., et al. 2010, ApJ, 720, 368

Yu, Q., \& Lu, Y. 2004, ApJ, 602, 603

Zel'dovich, Ya.B., \& Novikov, I.D. 1964, Sov. Phys. Dok., 9, 246

Zhao, D.H., Mo, H.J., Jing, Y.P., \& Börner, G. 2003, MNRAS, 339, 12

Zubovas, K., Nayakshin, S., Sazonov, S., \& Sunyaev, R. 2013, MNRAS, 431, 793

Zubovas, K., \& King, A. 2013, ApJ, 769, 51 


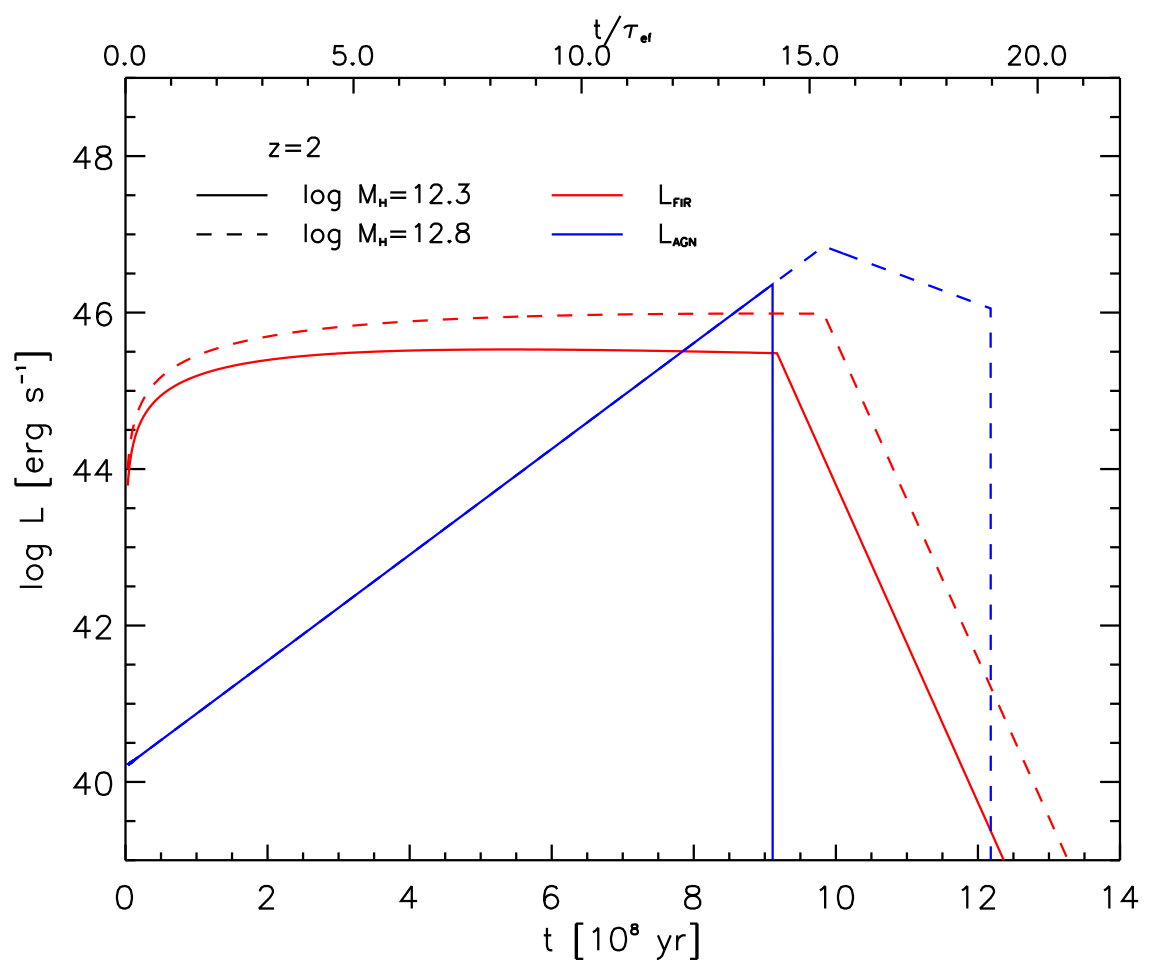

Figure 1. Evolution of the bolometric AGN luminosity due to accretion (blue lines) and of the FIR luminosity due to star formation (red lines) in galaxies with halo mass $M_{\mathrm{H}}=2 \times 10^{12} M_{\odot}$ (solid lines) and $M_{\mathrm{H}}=6 \times 10^{12} M_{\odot}$ (dashed lines) at redshift $z=2$. The lightcurves are plotted as a function of the galactic age in units of $10^{8} \mathrm{yr}$ (lower scale) and of the $e$-folding time $\tau_{\text {ef }} \approx 6 \times 10^{7} \mathrm{yr}$ (upper scale). 

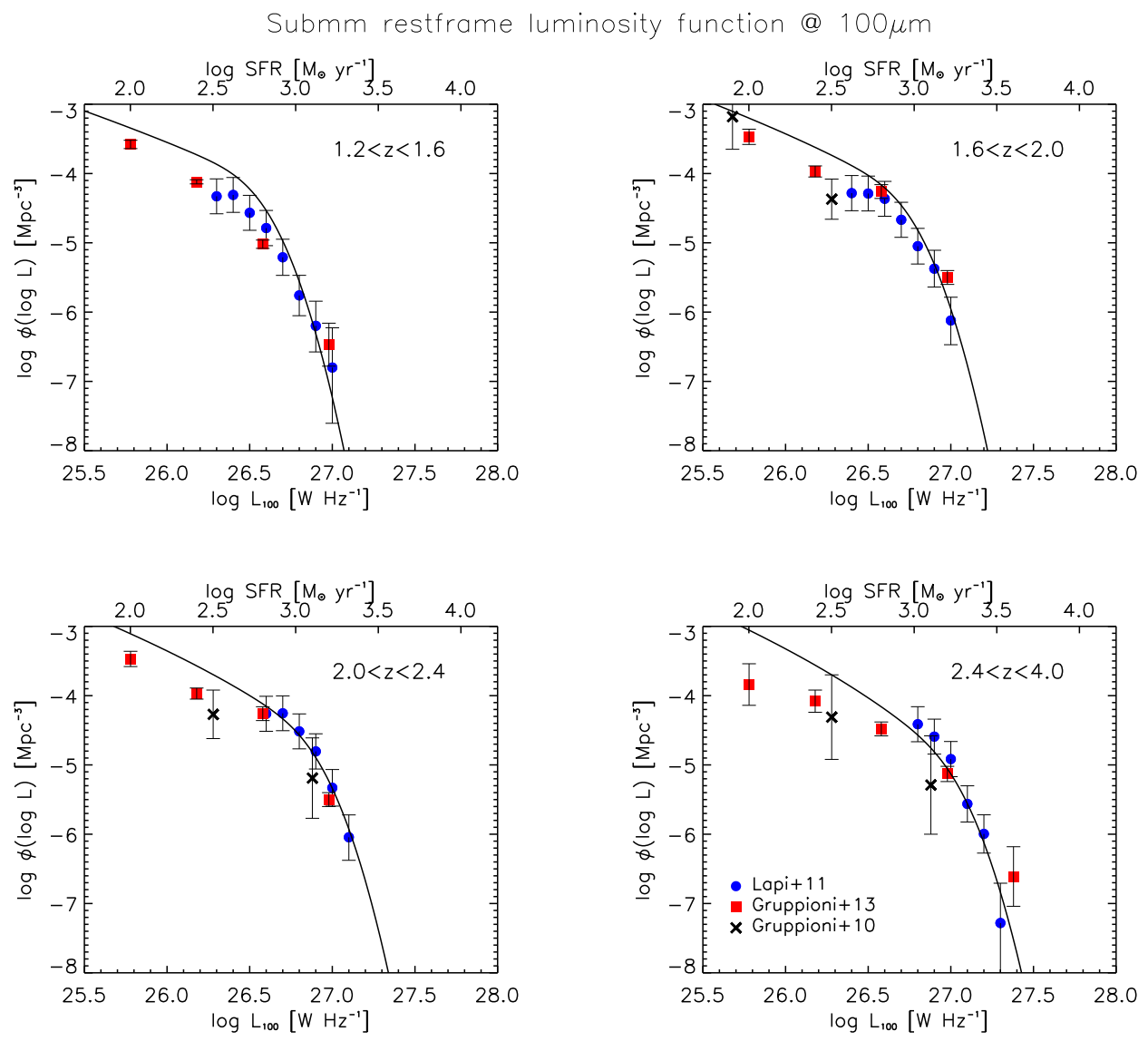

Figure 2. FIR luminosity function at a restframe wavelength of $100 \mu \mathrm{m}$. Each panel refers to a different redshift bin. The number density of sources is plotted as a function of the luminosity at $100 \mu \mathrm{m}$ restframe (lower axis) and of the SFR (upper axis), having adopted a Chabrier IMF and the SED of SMM J2135-0102, a typical high-redshift starbursting galaxy. The predictions of the model (solid lines) are compared with the observational data from the Herschel-ATLAS survey (Lapi et al. 2011) and the Herschel-PEP survey (Gruppioni et al. 2010, 2013) 


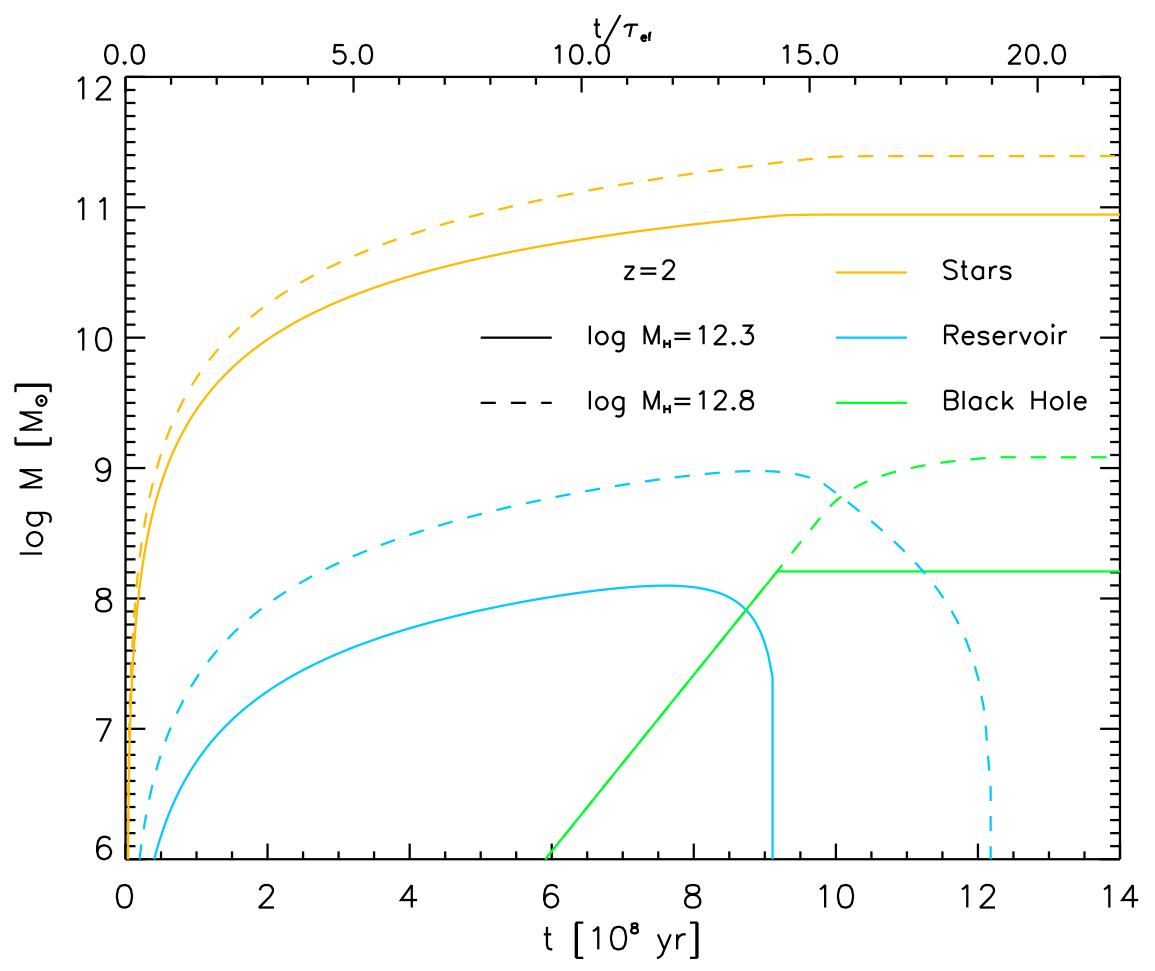

Figure 3. Same as Fig. 1, but for the evolution of the stellar mass (orange lines), reservoir mass (cyan lines), and BH mass (green lines). 


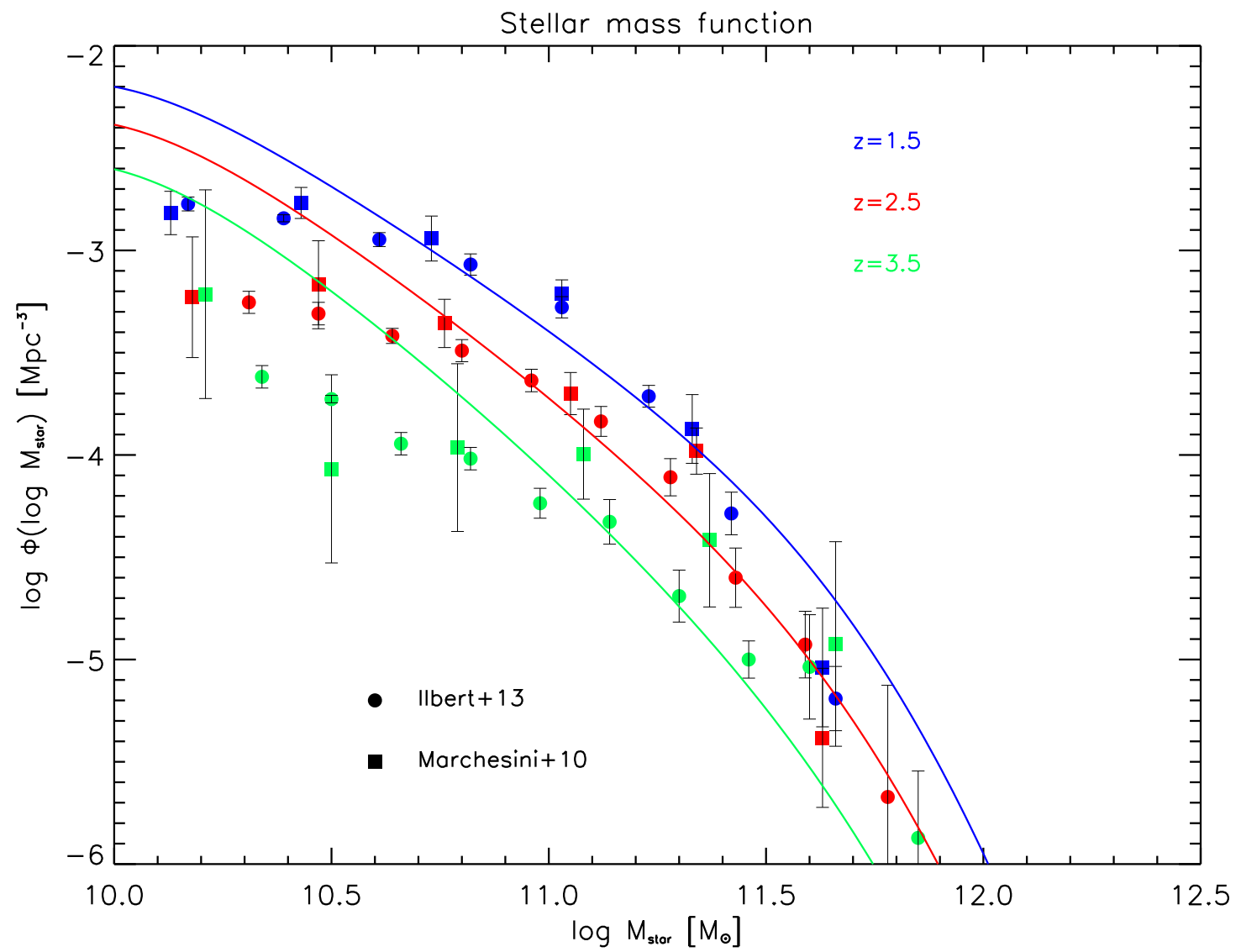

Figure 4. Stellar mass function of passively evolving ETGs at redshifts $z=1.5$ (blue), 2.5 (red), and 3.5 (green). Model predictions (solid lines) are compared to the observational data from Marchesini et al. (2010, squares) and Ilbert et al. (2013, circles). 


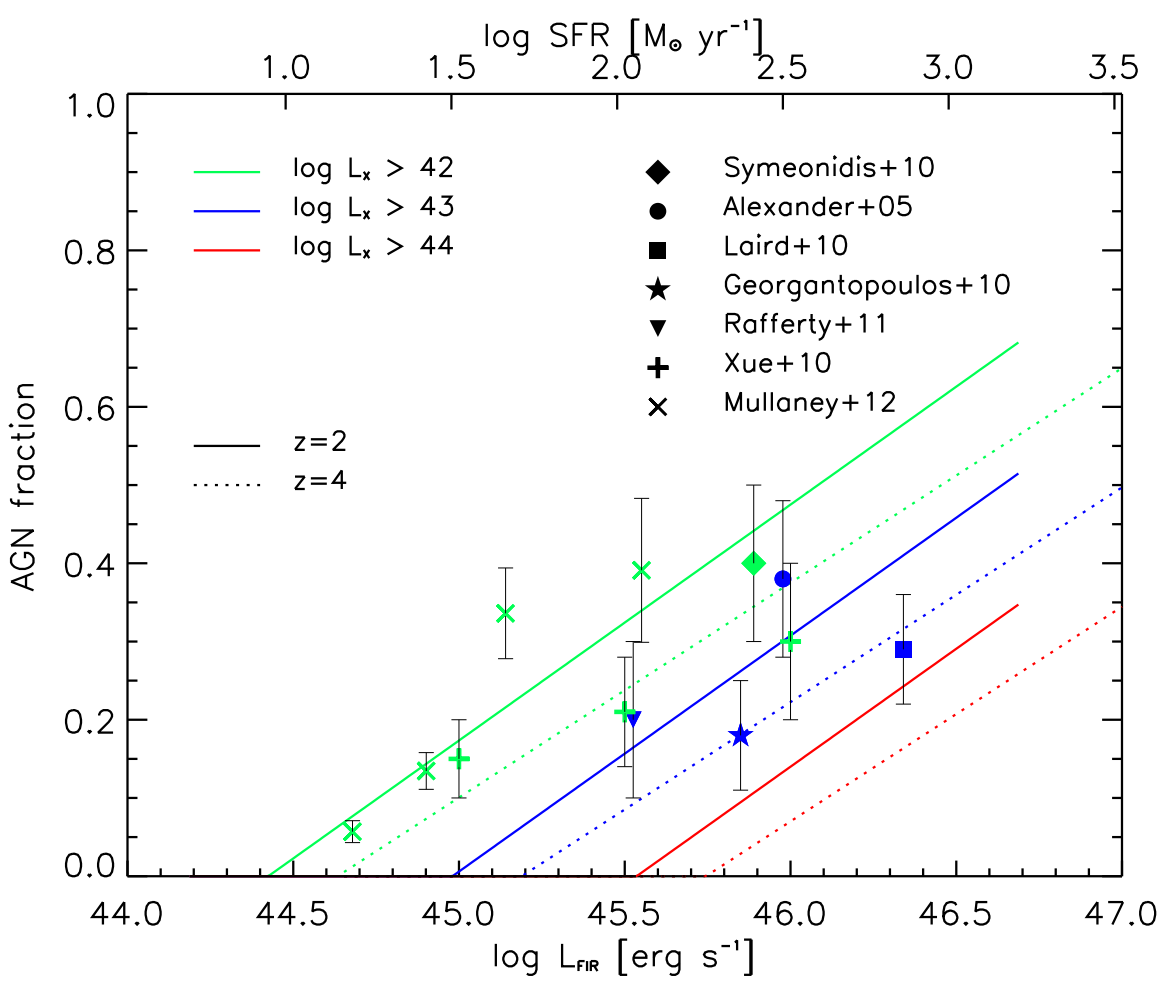

Figure 5. Fraction of X-ray detected AGNs in FIR/ $K$-band selected galaxies, as a function of the FIR luminosity (lower scale) and of the SFR (upper scale). Model predictions are provided for galaxies at $z=2$ (solid lines) and $z=4$ (dotted lines) for different X-ray detection thresholds $L_{X}=10^{42}$ (green), $10^{43}$ (blue), and $10^{44} \mathrm{erg} \mathrm{s}^{-1}$ (red) in the $2-10 \mathrm{keV}$ band; data (same color code) are from Alexander et al. (2005, circles), Georgantopoulos et al. (2011, stars), Laird et al. (2010, squares), Symeonidis et al. (2010; diamonds), Xue et al. (2010, plus signs), Rafferty et al. (2011, triangles), Mullaney et al. (2012a, crosses). 


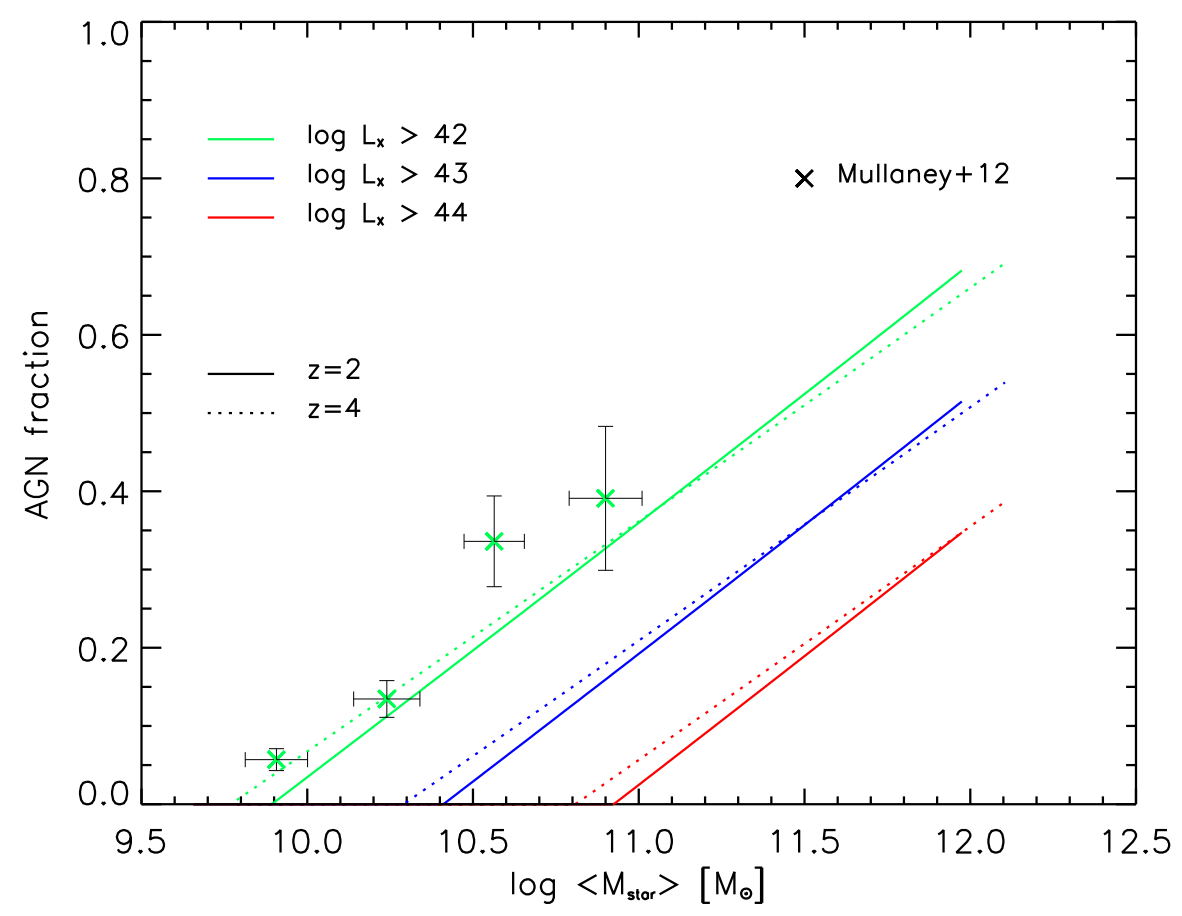

Figure 6. Fraction of X-ray detected AGN in $K$-band selected galaxies, as a function of the average stellar mass. Linestyles and color code as in Fig. 5. Data are from Mullaney et al. (2012a, crosses). 


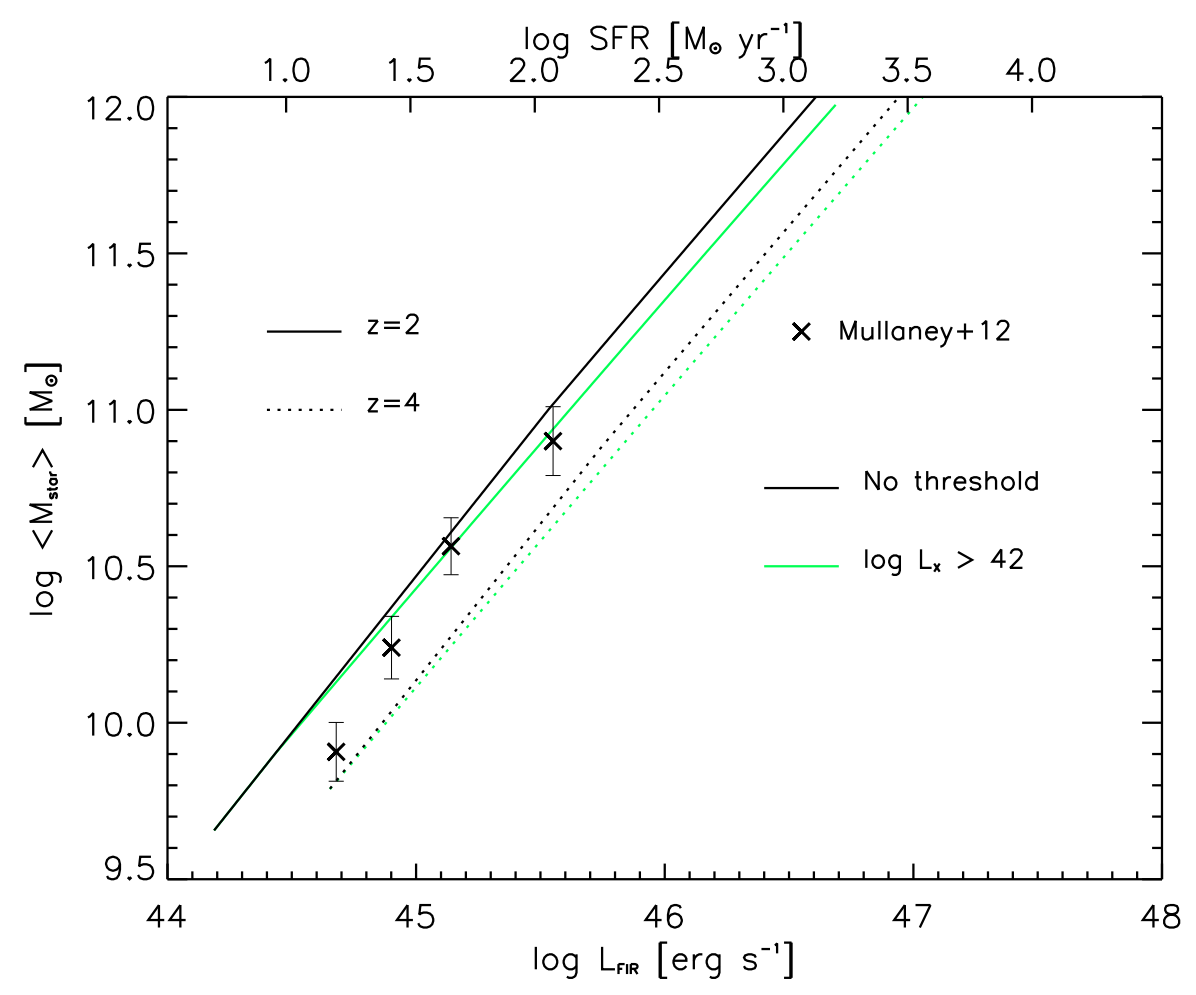

Figure 7. Average stellar mass in FIR/ $K$-band selected galaxies, as a function of the FIR luminosity (lower scale) and of the SFR (upper scale). Model predictions are provided at redshifts $z=2$ (solid lines) and $z=4$ (dotted lines), for an X-ray detection threshold $L_{X}=10^{42}$ $\mathrm{erg} \mathrm{s}^{-1}$ (green lines), and for no threshold (black lines); data (both for detected and undetected sources, with the former dominating) are from Mullaney et al. (2012a, crosses). 


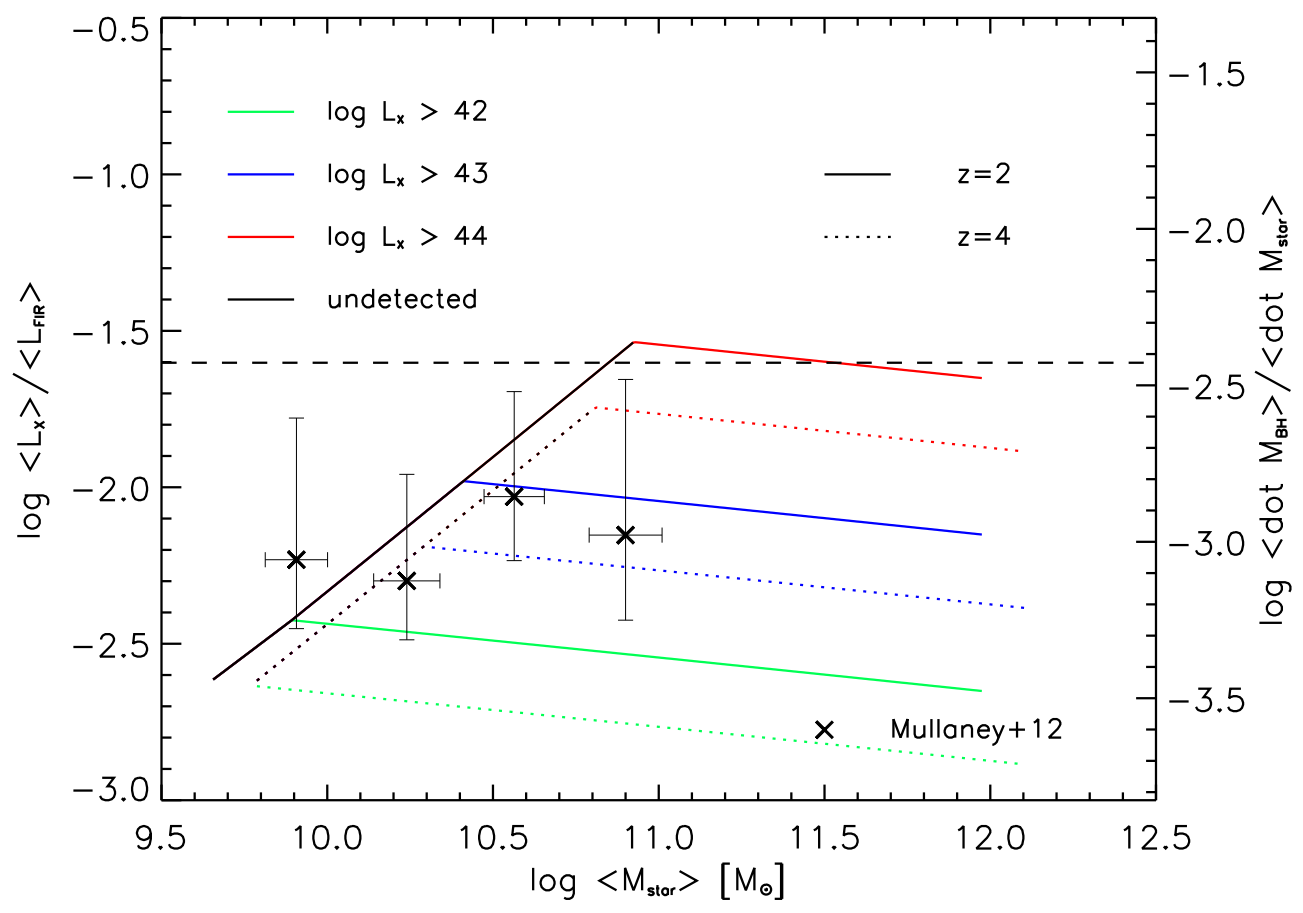

Figure 8. Ratios of average X-ray to FIR luminosity (left scale) and of average BH accretion rate to SFR (right scale) in $K$-band selected galaxies, as a function of the average stellar mass. Linestyles and color code are as in Fig. 5. The black lines show our predictions for undetected galaxies. The dashed line corresponds to $L_{\mathrm{FIR}}=L_{\mathrm{AGN}}$ adopting $k_{X}=40$ (see Hopkins et al. 2007). Data are from Mullaney et al. (2012a, crosses), and refer to a luminosity range a few $10^{42} \lesssim L_{X} \lesssim 10^{43} \mathrm{erg} \mathrm{s}^{-1}$. 

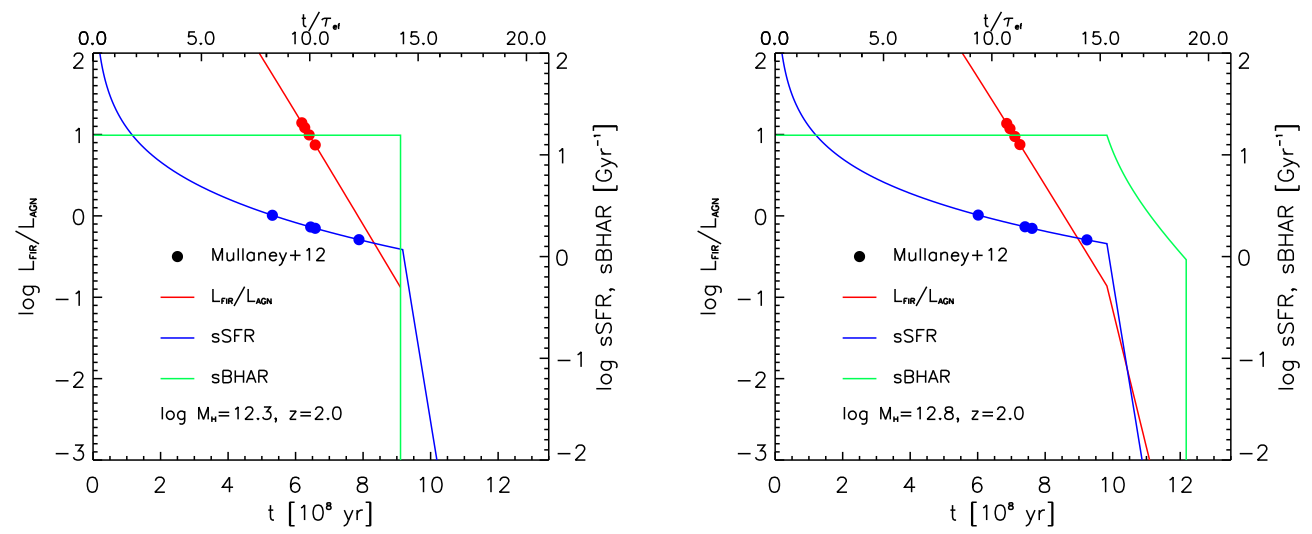

Figure 9. Evolution of the bolometric FIR to AGN luminosity ratio (red lines, left scale), of the specific SFR (blue lines, right scale), and of the specific $\mathrm{BH}$ accretion rate (green line, right scale) in galaxies with halo mass $M_{\mathrm{H}}=2 \times 10^{12} M_{\odot}\left(\right.$ left panel) and $M_{\mathrm{H}}=6 \times 10^{12} M_{\odot}$ (right panel) at redshift $z=2$; the quantities are plotted as a function of the galactic age in units of $10^{8}$ yr (lower scale) and of the $e$-folding time $\tau_{\mathrm{ef}} \approx 6 \times 10^{7} \mathrm{yr}$ (upper scale). The values of $L_{\mathrm{FIR}} / L_{\mathrm{AGN}}$ and of the sSFR observed by Mullaney et al. (2012a, dots) are marked onto the respective model predictions. 


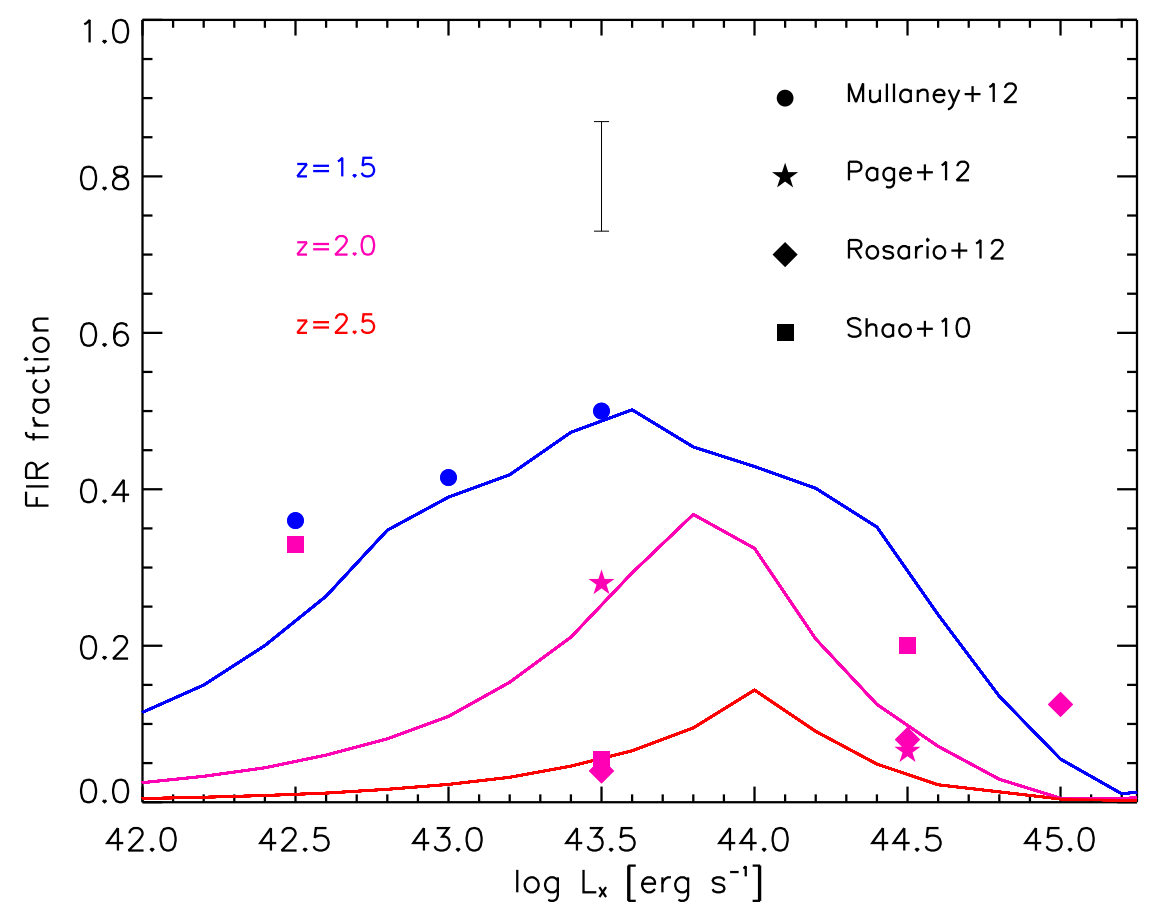

Figure 10. Fraction of FIR detected hosts in X-ray selected AGNs, as a function of the X-ray luminosity. Model predictions (solid lines) are provided at $z=1.5$ (blue), $z=2$ (magenta), and $z=2.5$ (red), for FIR detection thresholds $\log L_{\mathrm{FIR}}=44.8,45.3$, and 45.8 in erg $s^{-1}$, respectively. Data (same color code) are from Shao et al. 2010 (squares), Mullaney et al. (2012b, circles), Page et al. (2012, stars), and Rosario et al. (2012, diamonds); the typical error bar is also shown near the legend. 


$$
X \text {-ray } A G N \text { LF }
$$
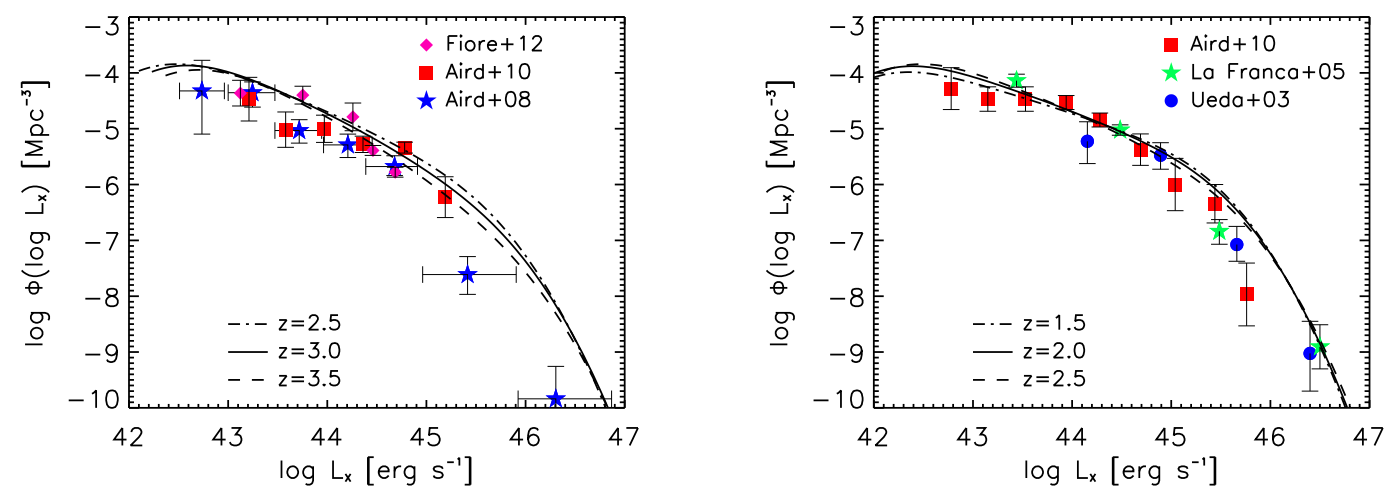

Figure 11. X-ray AGN luminosity function in two redshift bins centered around $z=2$ (right panel) and $z=3$ (left panel). Model predictions for various redshifts around these values (different linestyles) are compared with the observations by Ueda et al. (2003, blue circles), La Franca et al. (2005, green stars), Aird et al. (2008, blue stars), Aird et al. (2010, red squares), Fiore et al. (2012a, magenta diamonds). The X-ray bolometric correction by Hopkins et al. (2007) has been adopted. 


\section{Parameter dependence ( $X$-ray selected)}
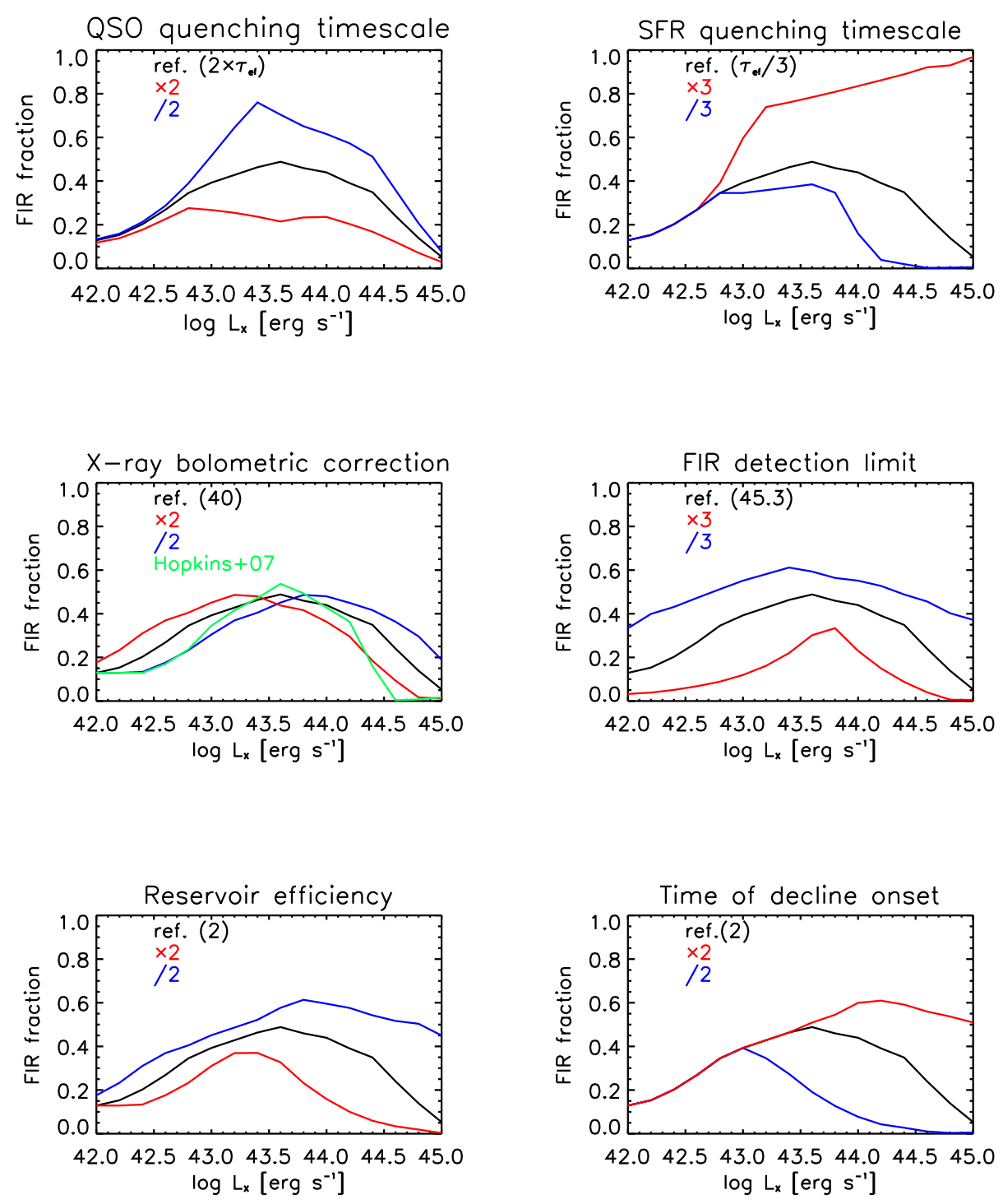

Figure 12. Dependence on various model parameters of the FIR detected fraction of X-ray selected AGNs at $z=2$ : quenching timescale of the AGN emission $\tau_{\mathrm{AGN}}$ (top left); quenching timescale of the SFR $\tau_{\mathrm{SFR}}$ (top right); X-ray bolometric corrections $k_{X}$ (middle left); FIR detection limit (middle right); reservoir efficiency $\alpha_{\text {res }}$ (bottom left); parameter $n$ determining the onset of the declining phase $t_{\mathrm{QSO}}+n \tau_{\text {ef }}$ (bottom right; see text for details). In each panel, three curves are shown: the black ones refer to a reference value of the parameter (reported in parenthesis in the legend), the red ones to an increased value, and the blue ones to a reduced value, as detailed in the figure. In the middle left panel, the additional green curve adopts the luminosity-dependent bolometric correction by Hopkins et al. (2007). 


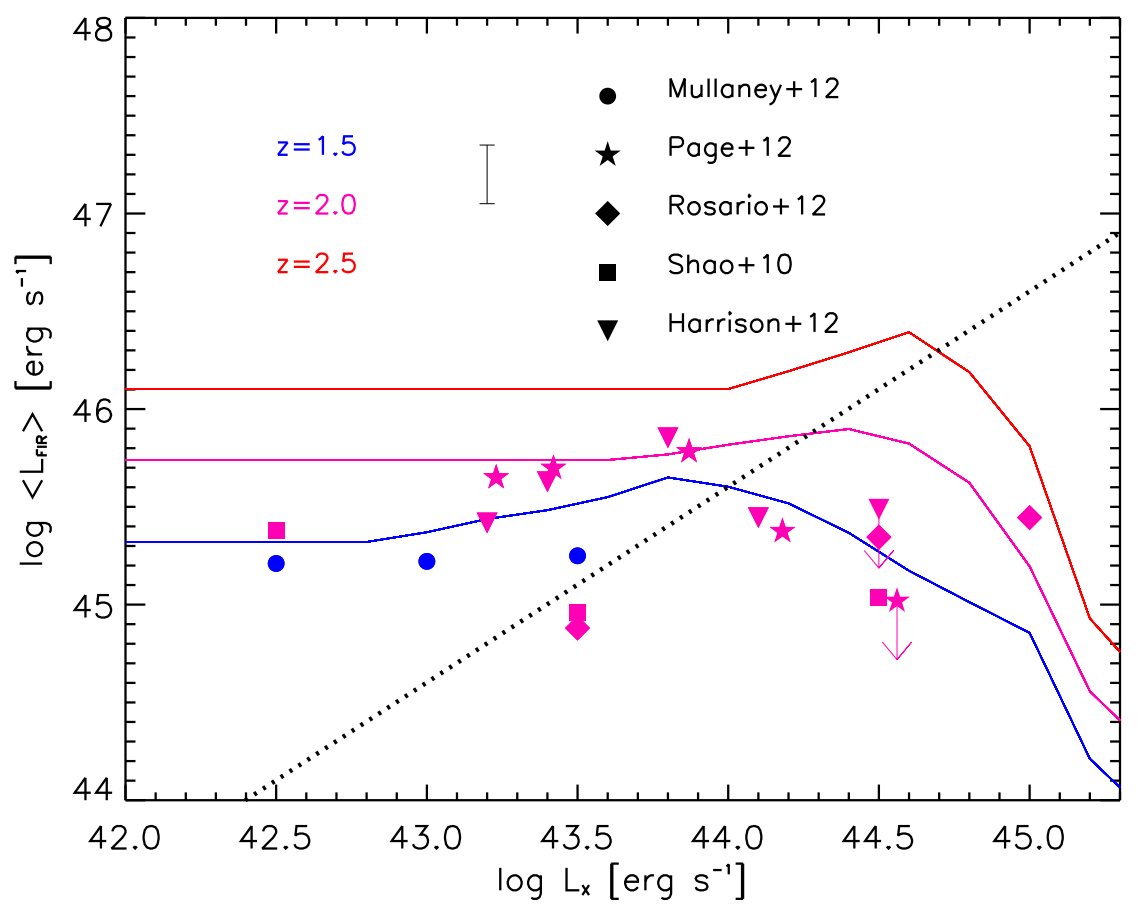

Figure 13. Average FIR luminosity of FIR detected hosts of X-ray selected AGNs, as a function of the X-ray luminosity. Linestyles and color code as in Fig. 10. Data are from Shao et al. (2010, squares), Harrison et al. (2012, triangles), Mullaney et al. (2012b, circles), Page et al. (2012, stars), and Rosario et al. (2012, diamonds). The dotted line corresponds to $L_{\mathrm{FIR}}=L_{\mathrm{AGN}}$ where a bolometric correction $k_{X}=40$ has been adopted. 


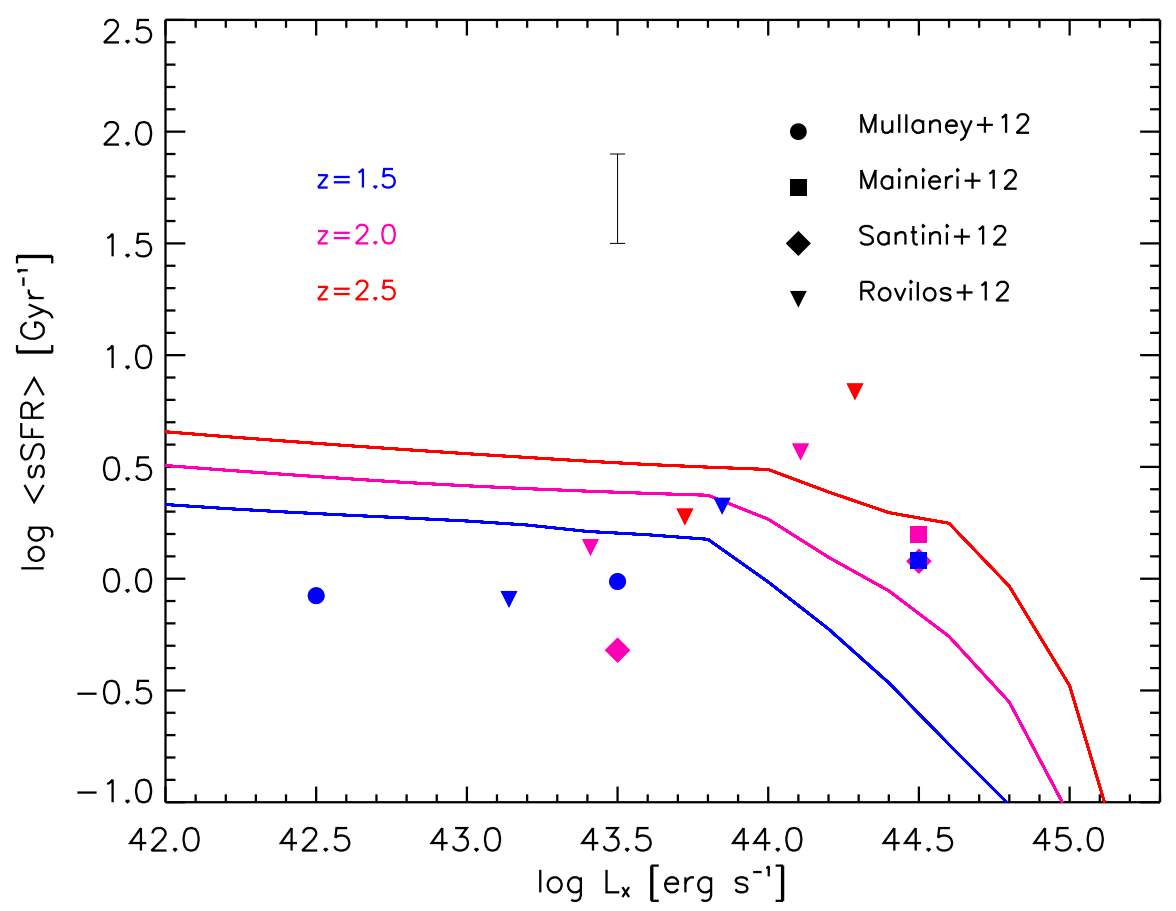

Figure 14. Average specific SFR of FIR detected hosts of X-ray selected AGNs, as a function of the X-ray luminosity. Linestyles and color-code as in Fig. 10. Data are from Mainieri et al. (2012, squares), Mullaney et al. (2012b, circles), Rovilos et al. (2012, triangles), and Santini et al. (2012, diamonds). 

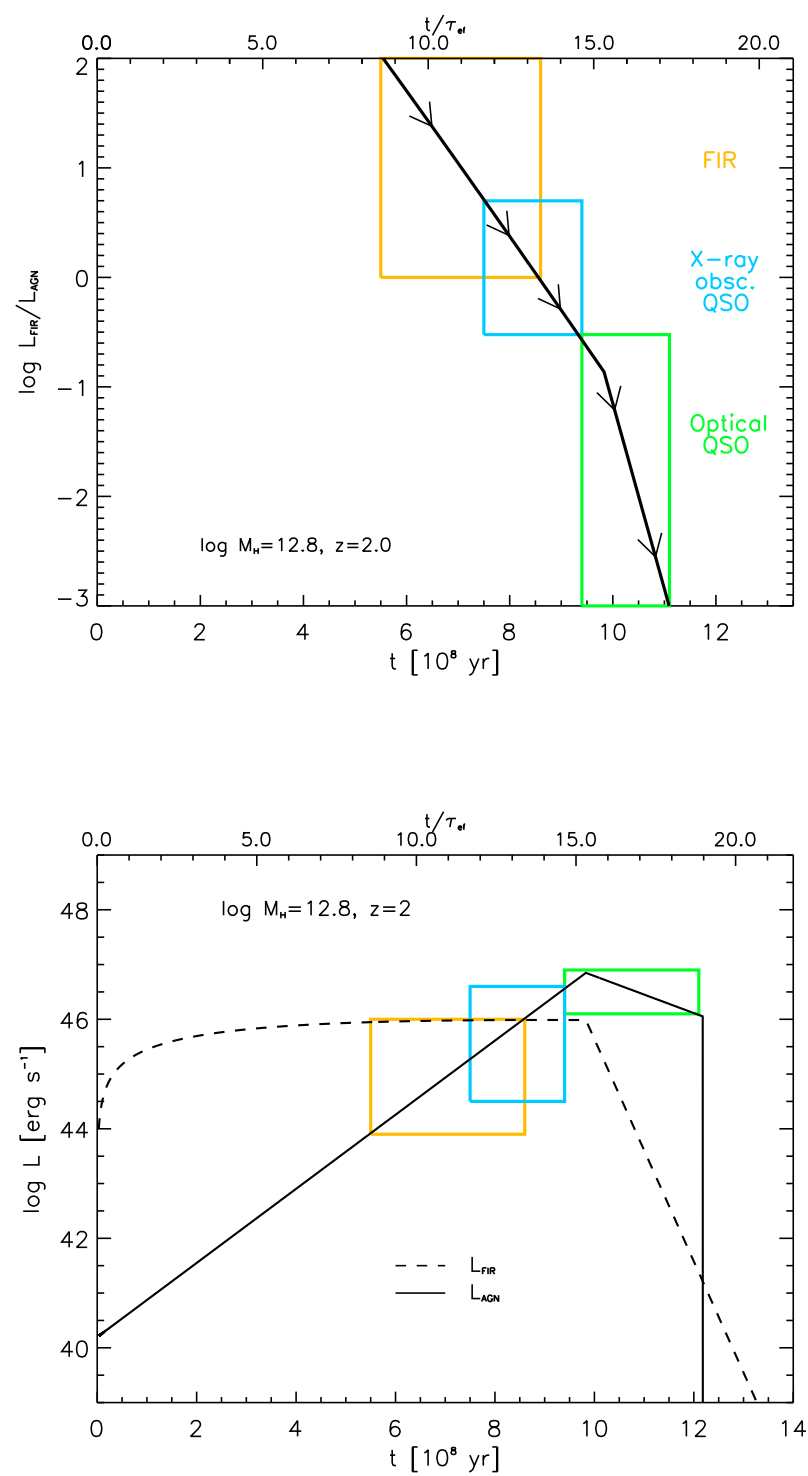

Figure 15. The top panel shows a schematic illustration of the different phases, marked by the colored boxes, in the galaxy/AGN coevolution envisaged by the model, as traced by the ratio $L_{\mathrm{FIR}} / L_{\mathrm{AGN}}$. Specifically, the orange box refers to the FIR bright phase, the cyan box to the obscured X-ray QSO phase, and the green box to the optically bright QSO phase. Note also that an X-ray selection unbiased to obscuration can pick up objects with very different values of $L_{\mathrm{FIR}} / L_{\mathrm{AGN}}$, cf. Fig. 13 . The bottom panel shows the placement of the same phases on the FIR and AGN lightcurves. 


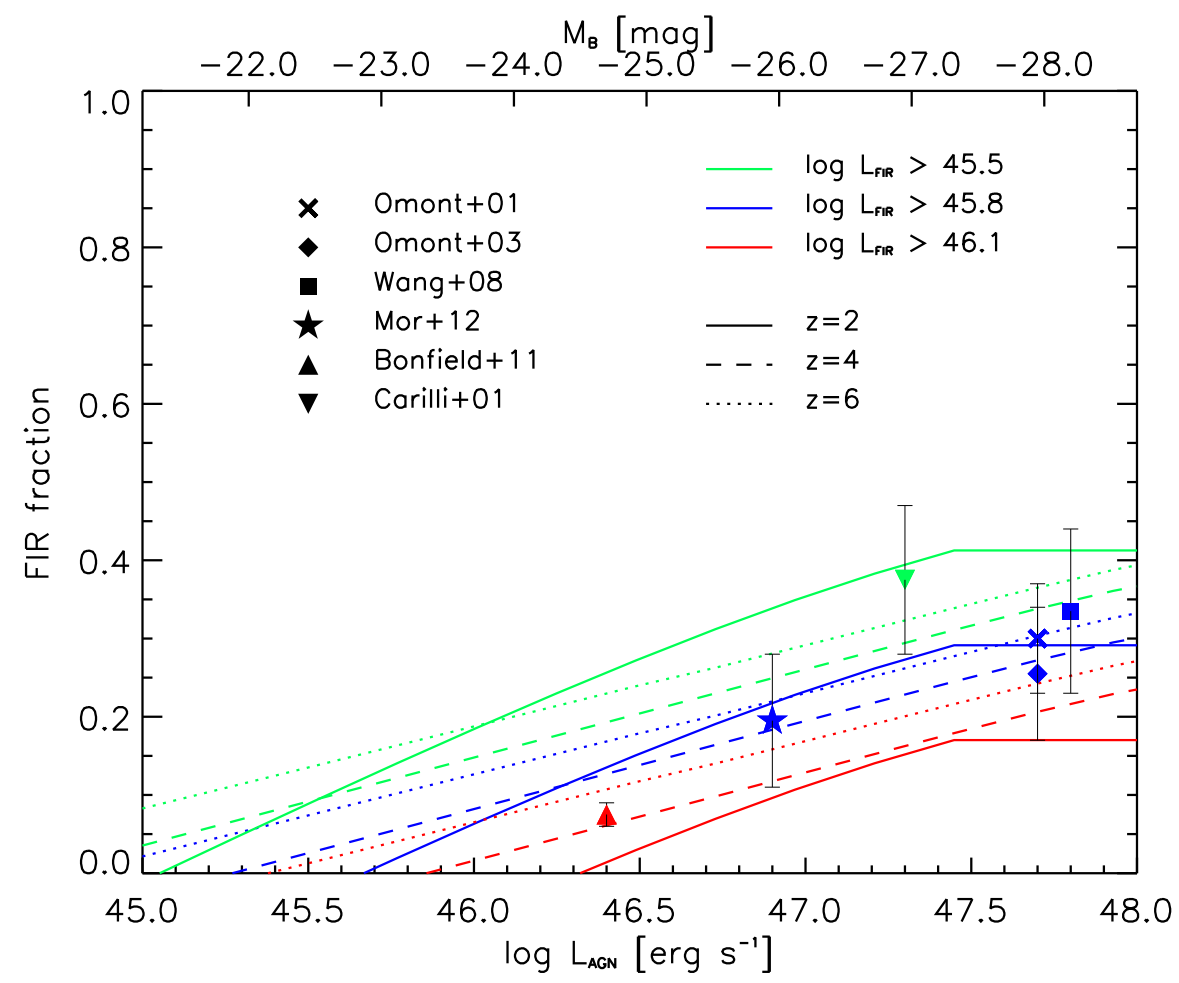

Figure 16. Fraction of FIR detected hosts of optically selected AGNs, as a function of the AGN bolometric luminosity (lower scale) and of the $B$-band magnitude (upper scale). Model predictions are provided at redshift $z=2$ (solid lines), 4 (dashed lines), and 6 (dotted lines), for different values of the FIR detection threshold $\log L_{\mathrm{FIR}}=45.5$ (green lines), 45.8 (blue lines), and 46.1 (red lines) in units of erg $\mathrm{s}^{-1}$. Data (same color code) are from Carilli et al. (2001, reversed triangle), Omont et al. (2001, cross), Omont et al. (2003, diamond), Wang et al. (2008, square), Bonfield et al. (2011, triangle), and Mor et al. (2012, star). 
Parameter dependence (optically selected)
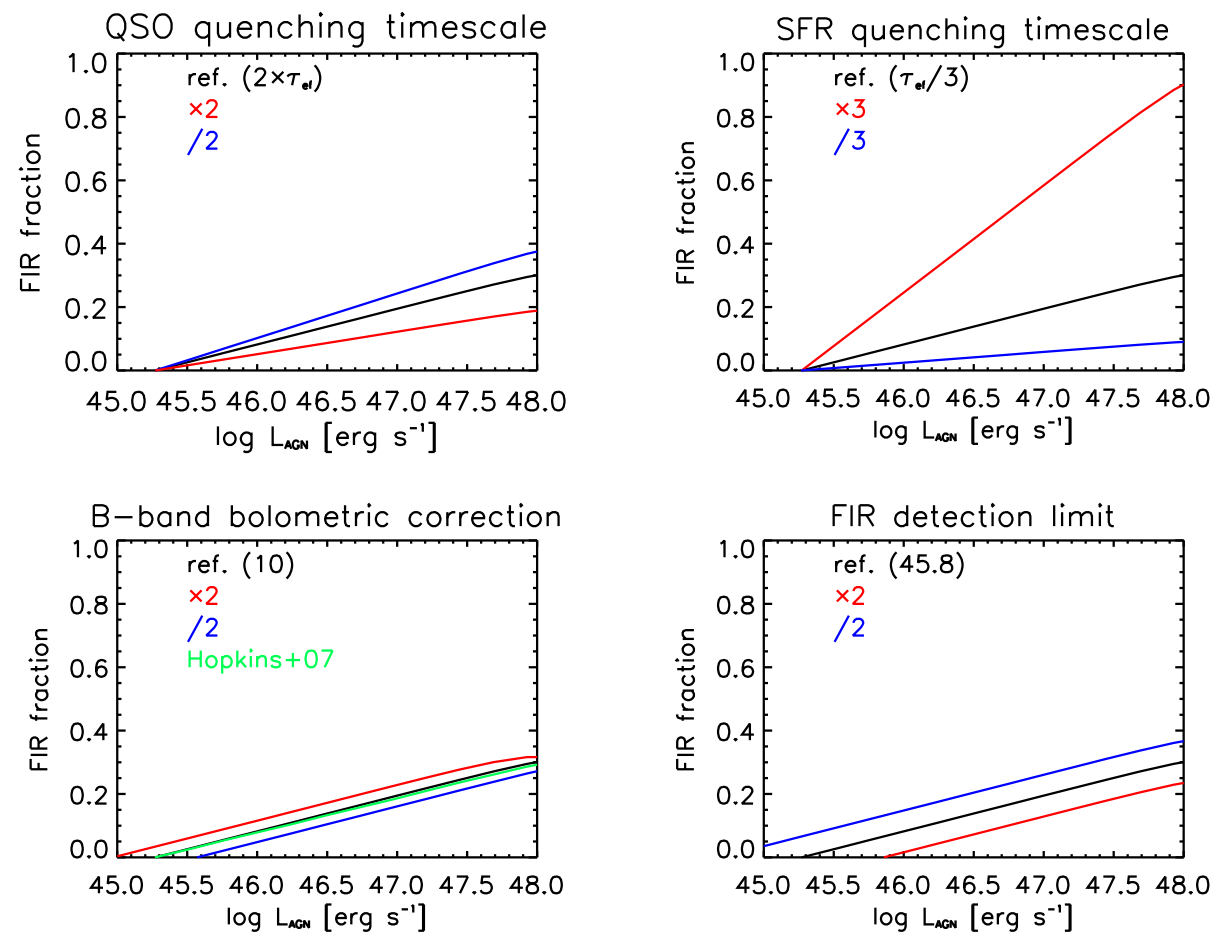

Figure 17. Dependence on various model parameters of the FIR detected fraction of optically selected AGNs at $z=4$ : quenching timescale of the AGN $\tau_{\mathrm{AGN}}$ (top left); quenching timescale of the SFR $\tau_{\mathrm{SFR}}$ (top right); X-ray bolometric corrections $k_{X}$ (bottom left); FIR detection threshold (bottom right). In each panel, three curves are shown: the black ones refer to the fiducial value of the parameter (reported in parenthesis), the red ones to an increased value, and the blue ones to a reduced value, as detailed in the legend. In the bottom left panel, the additional green curve is for the luminosity-dependent bolometric correction by Hopkins et al. (2007). 


\section{Optical AGN LF}
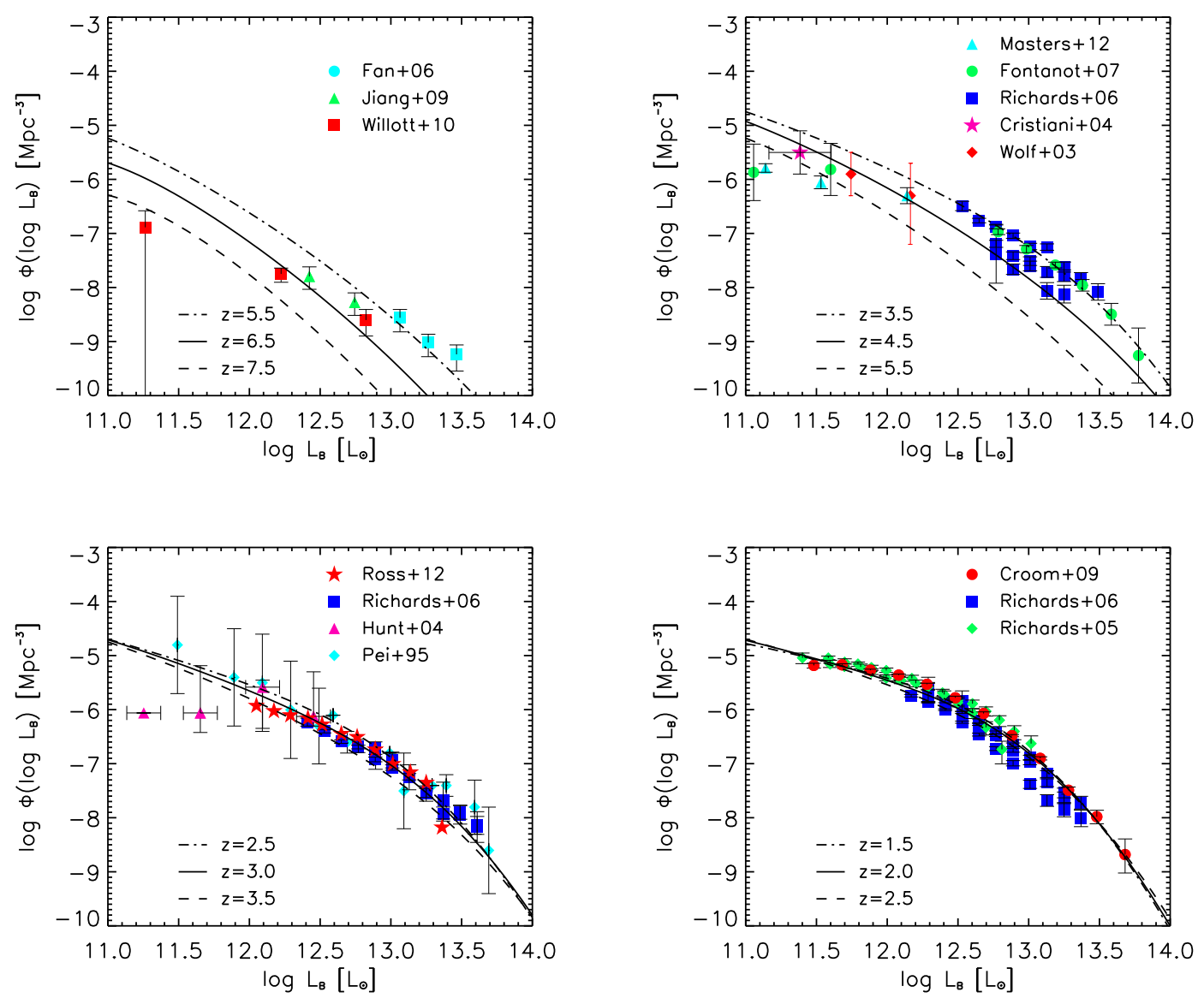

Figure 18. Optical QSO luminosity function in four redshift bins centered around $z=6.5$ (top left), 4.5 (top right), 3 (bottom left), and 2 (bottom right). Model predictions for various redshifts around these values (different linestyles) are compared with the observations by Pei (1995, cyan diamonds), Wolf et al. (2003, red diamonds), Cristiani (2004, magenta star), Hunt et al. (2004, magenta triangles), Richards et al. (2005, green diamonds), Fan et al. (2006, cyan circles), Richards et al. (2006, blue squares), Fontanot et al. (2007, green circles), Croom et al. (2009, red circles), Jiang et al. (2009, green triangles), Willott et al. (2010, red squares), Masters et al. (2012, cyan triangles), Ross et al. (2013, red stars). The optical bolometric correction by Hopkins et al. (2007) has been adopted. 


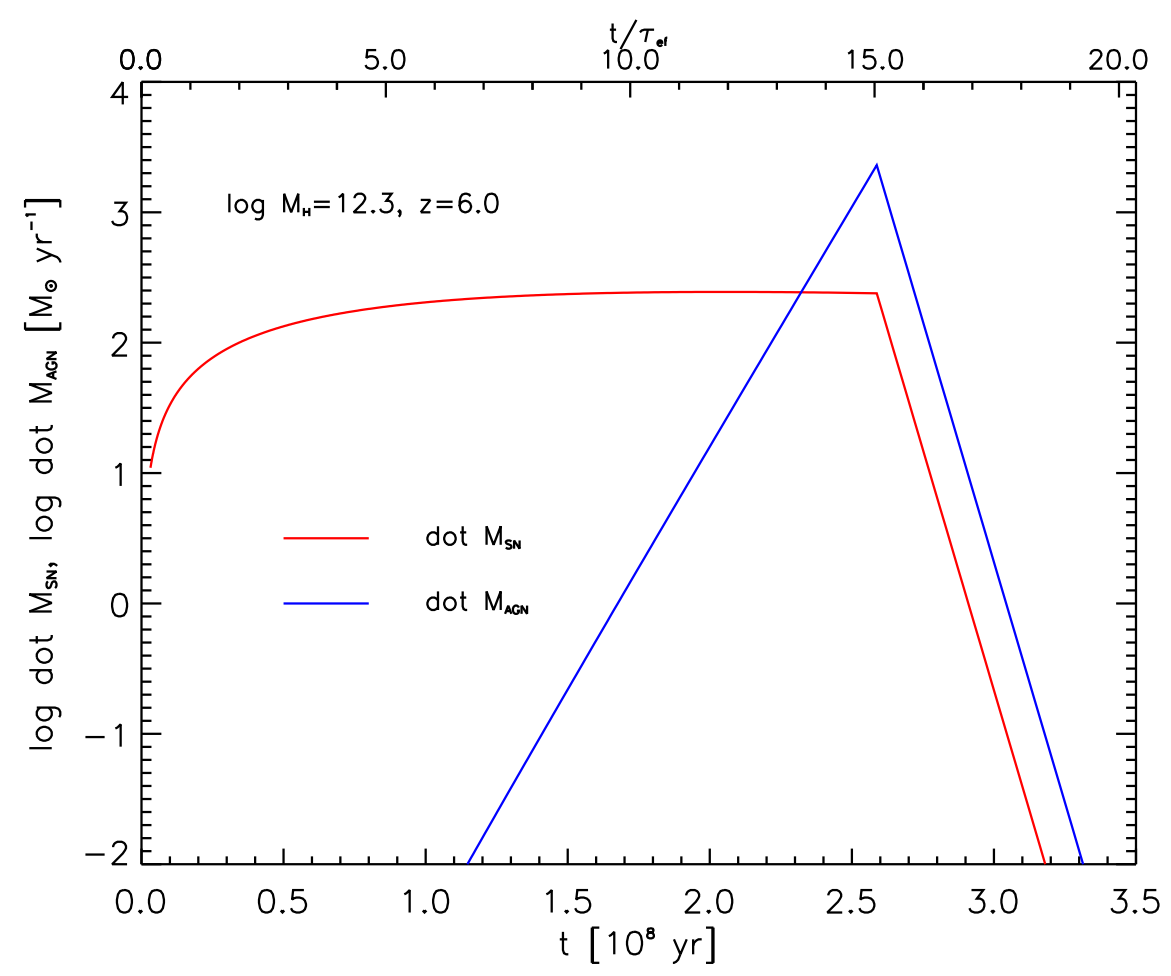

Figure 19. Evolution of the cold gas outflow rate due to SN (red line) and AGN feedback (blue lines) for a galaxy with halo mass $M_{\mathrm{H}}=2 \times 10^{12} M_{\odot}$ at redshift $z=6$; the curves are plotted as a function of the galactic age in units of $10^{8} \mathrm{yr}$ (lower scale) and of the $e$-folding time $\tau_{\text {ef }}=2 \times 10^{7}$ yr (upper scale). 


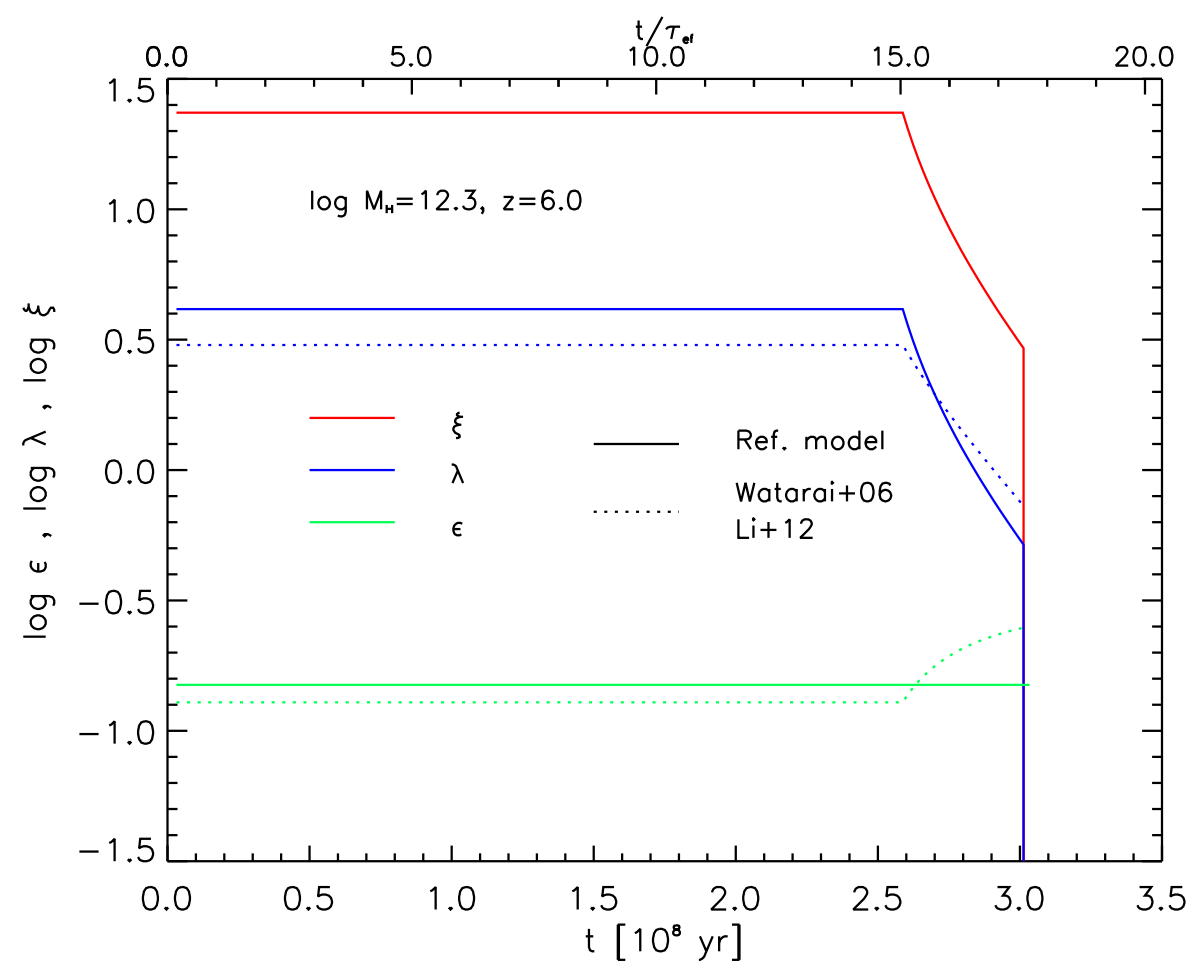

Figure 20. Evolution of the ratio between the BH mass accretion to the Eddington rate $\xi=\dot{M}_{\text {accr }} c^{2} / L_{\text {Edd }}$ (red line), of the Eddington ratio $\lambda=L / L_{\mathrm{Edd}}$ (blue line), and of the radiative efficiency $\epsilon=\lambda / \xi$ (green line) in a galaxy with halo mass $M_{\mathrm{H}}=2 \times 10^{12} M_{\odot}$ at redshift $z=6$; the evolution curves are plotted as a function of the galactic age in units of $10^{8} \mathrm{yr}$ (lower scale) or of the $e$-folding time $\tau_{\text {ef }}=2 \times 10^{7}$ yr (upper scale). The solid lines refer to the model while the dotted lines to the prescriptions by Watarai (2006) and Li (2012). 